\title{
Transfer Learning for Fuzzy Actor-Critic Learning via Fuzzy Rule Transfer
}

\author{
by \\ Dawei Ni, B.Eng. \\ A thesis submitted to the \\ Faculty of Graduate and Postdoctoral Affairs \\ in partial fulfillment of the requirements for the degree of
}

Master of Applied Science in Electrical and Computer Engineering

Ottawa-Carleton Institute for Electrical and Computer Engineering

Department of Systems and Computer Engineering

Carleton University

Ottawa, Ontario

Aug, 2020

(C) Copyright

Dawei Ni, 2020 
The undersigned hereby recommends to the

Faculty of Graduate and Postdoctoral Affairs acceptance of the thesis

\title{
Transfer Learning for Fuzzy Actor-Critic Learning via Fuzzy Rule Transfer
}

\author{
submitted by Dawei Ni, B.Eng. \\ in partial fulfillment of the requirements for the degree of
}

Master of Applied Science in Electrical and Computer Engineering

Professor Howard Schwartz, Thesis Supervisor

Professor James Green, Chair,

Department of Systems and Computer Engineering

Ottawa-Carleton Institute for Electrical and Computer Engineering

Department of Systems and Computer Engineering

Carleton University

Aug, 2020 


\section{Abstract}

Reusing knowledge from previous experience to accelerate learning is the central idea in transfer learning. This notion also applies to reinforcement learning where the agents acquire knowledge via interacting with the environment. In this thesis, we explore transfer learning in the fuzzy reinforcement learning domain, particularly in the environment of differential games. We present a novel approach to transfer knowledge between similar tasks which use the Fuzzy Actor Critic Learning (FACL) algorithm. Specifically, we propose a fuzzy rule transfer (FRT) method to map fuzzy rules between source and target tasks. The target task is assumed to be related to the source task but with more complex states. Our method is fully implemented and tested in the domain of differential games where all state space and action space are

continuous. The simulation results show that agents are able to learn and achieve asymptotic performance faster in the target task when the FRT technique is applied. 


\section{Acknowledgments}

I am extremely grateful to my thesis supervisor Prof. Howard Schwartz for his great guidance and support throughout my thesis studies. He has been patient, caring and providing great advice to me since the beginning of my master program. The milestone of this master thesis would not have been achieved without him.

I also want to give my gratitude to the professors, staff in Carleton university, particularly in the department of system and computer engineering for their support.

Finally, I would like to express my gratitude to my family for their endless support, love, and encouragement. 


\section{Table of Contents}

Abstract iii

Acknowledgments $\quad$ iv

Table of Contents $\quad$ v

List of Figures $\quad$ vii

1 Introduction 1

1.1 Overview . . . . . . . . . . . . . . . . . . . . . . 1

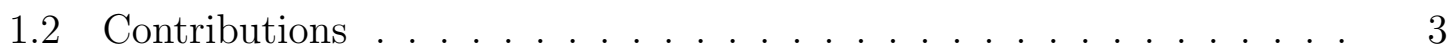

1.3 Thesis Organization . . . . . . . . . . . . . . . . . . 4

2 Reinforcement Learning and Fuzzy Reinforcement Learning 5

2.1 Markov Decision Process . . . . . . . . . . . . . . . . 7

2.2 Reward and Return . . . . . . . . . . . . . . . . . . 9

2.3 Value Function and Policy . . . . . . . . . . . . . . . 10

2.4 Temporal-Difference Learning . . . . . . . . . . . . . . . . . . . 12

2.4 .1 TD Prediction . . . . . . . . . . . . . . . . . . 12

2.4 .2 Q-learning . . . . . . . . . . . . . . . . . . . . 13

2.5 Fuzzy Rules and Fuzzy systems _ . . . . . . . . . . . . . 15 
2.6 Fuzzy Actor Critic Learning . . . . . . . . . . . . . . . . . . . . . . . 18

2.7 Summary . . . . . . . . . . . . . . . . . 23

3 Transfer Learning $\quad 24$

3.1 Transfer Learning Overview . . . . . . . . . . . . . 26

3.1.1 Evaluating Transfer Learning Methods . . . . . . . . . . . . . 27

3.1.2 The Five Dimensions of Transfer Learning . . . . . . . . . . . 29

3.2 Transfer Learning via Inter-Task Mappings . . . . . . . . . . . . . . . 31

3.2.1 TVITM for Keepaway Game . . . . . . . . . . . . . . . . . . . 32

3.2.2 Transfer Functional Structure . . . . . . . . . . . . . . . 37

3.2.3 TVITM for Different Function Approximators . . . . . . . . . 38

3.3 Transfer via InterTask Mappings in Policy Search . . . . . . . . . . . 42

3.4 Transfer Learning Example in the Paddle Game . . . . . . . . . . . . 43

3.5 Summary . . . . . . . . . . . . . . . . 50

4 Transfer Learning in the FACL Algorithm 51

4.1 Fuzzy Rule Transfer in FACL . . . . . . . . . . . . . . . . . 52

4.1.1 Similarity between Fuzzy Rules . . . . . . . . . . . . . 54

4.1.2 Fuzzy Rule Transfer Example . . . . . . . . . . . . . . . . 55

4.2 Simulation and Result . . . . . . . . . . . . . . . 58

4.2.1 Pursuit-Evasion Game . . . . . . . . . . . . . . . . . . . 59

4.2.2 Guarding a Territory Game . . . . . . . . . . . . . . 74

4.3 Summary . . . . . . . . . . . . . . . . 83

5 Conclusion $\quad 85$

$\begin{array}{ll}\text { List of References } & 88\end{array}$ 


\section{List of Figures}

2.1 The agent-environment interaction in reinforcement learning . . . . . 7

2.2 Basic configuration of pure fuzzy systems f . . . . . . . . . . 16

2.3 Basic configuration of Takagi-Sugeno-Kang fuzzy system . . . . . . . 17

2.4 Basic configuration of fuzzy systems with fuzzifier and defuzzifier. . . 18

2.5 The fuzzy actor critic learning structure. . . . . . . . . . . . . . 20

2.6 Membership function for input of distance. . . . . . . . . . . . . . . 21

3.1 Total Time Scenario and Target Task Time scenario in TF learning . 27

3.2 Various performance metrics used in TF learning . . . . . . . . . . 29

3.3 The $\rho$ transfer function between tasks . . . . . . . . . . . . . 33

3.4 The game environment of Keepaway . . . . . . . . . . . . . . . 34

3.5 The game environment of Keepaway . . . . . . . . . . . . . . 35

3.6 Mapping between states in 4 vs. 3 to states in 3 vs. 2. . . . . . . . 36

3.7 The use of mapping $\chi_{x}$ and $\chi_{a}$ in $\rho \ldots \ldots . \ldots . \ldots 38$

3.8 13-20-3 Neural Network structure in the source task . . . . . . . . . . 39

3.9 19-20-4 Neural Network structure in the target task . . . . . . . . . . 40

3.10 Paddle game with 1 ball . . . . . . . . . . . . . . . . . 44

3.11 Paddle game with 2 balls . . . . . . . . . . . . . . 45

3.12 Reward per episode between $\mathrm{TF}$ and non-TF case . . . . . . . . . . . 47

3.13 Cumulative hits between $\mathrm{TF}$ and non-TF case . . . . . . . . . . 48 
$3.14 \mathrm{NN}$ weights with $\mathrm{TF} \ldots \ldots \ldots \ldots \ldots$

$3.15 \mathrm{NN}$ weights without $\mathrm{TF} \ldots \ldots \ldots \ldots$

4.1 Game trajectory 1 evader vs. 1 pursuer with the time penalty constant and the evader escapes . . . . . . . . . . . . . 61

4.2 Game trajectory 1 evader vs. 1 pursuer without the time penalty constant and the evader escapes . . . . . . . . . . . . . 62

4.3 Game trajectory 1 evader vs. 3 pursuer . . . . . . . . . . . . . 62

4.4 Average reward using FRT in 1 evader vs. 3 pursuers game (only the evader is learning $\ldots \ldots \ldots \ldots \ldots \ldots \ldots$

4.5 Average reward without Transfer in 1 evader vs. 3 pursuers game (only the evader is learning $\ldots \ldots \ldots \ldots \ldots \ldots$

4.6 Epoch 500 using FRT in 1 evader vs. 3 pursuers game (only the evader

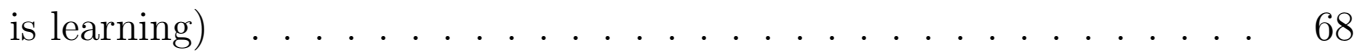

4.7 Epoch 500 without FRT in 1 evader vs. 3 pursuers game (only the evader is learning $\quad \ldots \ldots \ldots \ldots$

4.8 High speed evader E1 chased by three pursuers, P1, P2 and P3 . . . 70

4.9 Geometric illustration of capture condition . . . . . . . . . . 70

4.10 Epoch 600 without FRT in multi-agent 1 evader vs. 3 pursuers game when all agents are learning . . . . . . . . . . . . . 72

4.11 Epoch 600 with FRT in multi-agent 1 evader vs. 3 pursuers game when all agents are learning $\ldots \ldots \ldots \ldots \ldots \ldots \ldots \ldots$

4.12 Consequent parameters for evader in 1 evader vs. 3 pursuers game when all agents are learning with FRT . . . . . . . . . . 74

4.13 Consequent parameters for evader in 1 evader vs. 3 pursuers game when all agents are learning without FRT . . . . . . . 75 
4.14 Consequent parameters for pursuer1 in 1 evader vs. 3 pursuers game when all agents are learning with FRT . . . . . . . . . . . . 76

4.15 Consequent parameters for pursuer1 in 1 evader vs. 3 pursuers game when all agents are learning without FRT . . . . . . . . . . . 76

4.16 Game trajectory 1 invader vs. 1 guard in Territory game as the source

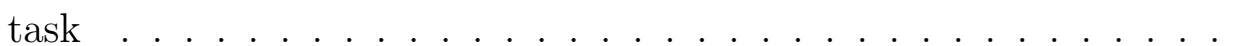

4.17 Game trajectory 1 invader vs. 2 guard in Territory game as the target

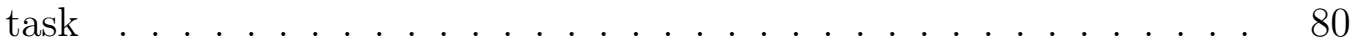

4.18 Simulation time-steps in 1 invader vs. 2 guards game when all agents are learning with FRT . . . . . . . . . . . . . . . .

4.19 Simulation time-steps in 1 invader vs. 2 guards game when all agents are learning without FRT . . . . . . . . . . . . . .

4.20 Epoch 300 in 1 invader vs. 2 guards game when all agents are learning with FRT . . . . . . . . . . . . . . . . . . . . . 82

4.21 Epoch 300 in 1 invader vs. 2 guards game when all agents are learning without FRT . . . . . . . . . . . . . . . . 


\section{Chapter 1}

\section{Introduction}

\subsection{Overview}

Reinforcement learning(RL) provides a framework for an agent to learn how to map situations to actions so as to maximize a numerical reward signal [1]. The learner is not given any information on which actions to take, instead it must discover which actions yield the most reward by exploring those actions in the environment. Actions may affect not only the immediate reward but also the next situation and all subsequent rewards [1]. The reward is in the form of a numerical value, which is generated amid the interaction between agent and environment. The agent's learning process is thus guided by the reward, and with the goal of finding a policy that maximizes the total amount of the reward it receives over the long run [1].

In reinforcement learning, if the state and action spaces are discrete and small, we can store the policy in a look-up table. However, if the state and action spaces are in the continuous domain, which means there is an infinite number of states and actions to evaluate, it is intractable to maintain such a look-up table. To deal with this situation, function approximation can be used to represent the continuous state and action spaces in an effective way [2], [3]. We focus on one of the widely used 
function approximators, the fuzzy logic controller (FLC), in this study. FLCs are universal approximators that can estimate any continuous function to any degree of accuracy [4]. Reinforcement learning combined with FLCs can be used to solve problems in the continuous domain. When reinforcement learning is associated with FLCs, the approach is commonly called fuzzy reinforcement learning [5].

While reinforcement learning is gaining significant attention in the artificial intelligent area, problems arise when mastering complex tasks due to slow convergence to a good policy. For instance, temporal-difference(TD) learning methods [1] are a type of reinforcement learning that has shown some success in different machine learning tasks because of its ability to learn where there is limited prior knowledge and minimal environmental feedback [6]. However, TD is often very slow in practice to produce near-optimal behaviors. Many techniques exist which attempt, with more or less success, to speed up the learning process [6]. Thus, a large amount of research has been targeted at improving the learning speed of agents, which includes using transfer learning in RL. The insight behind transfer learning is that generalizations may occur not only within tasks, but also across tasks [7]. The idea is similar to the human learning process; we use our previous experience to efficiently tackle new tasks. Similarly, the way of learning can also benefit the RL agents given the appropriate experience from a similar task they have learned previously. In the reinforcement learning context, we aim to transfer knowledge acquired by the agent from a previous task (source task) to a new task (target task) in order to speed up learning.

In this thesis, we investigate the possibility of using transfer learning in the fuzzy reinforcement learning domain. A novel approach is proposed to transfer knowledge among similar tasks that use fuzzy actor-critic learning (FACL) algorithm. We also introduce a fuzzy rule transfer (FRT) method specifically for processing the mapping of the fuzzy rules among the source and target tasks. We make an assumption that 
the target task is related to the source task but is represented by more complex states. Our method is fully implemented and tested in the domain of differential games where all state spaces and action spaces are continuous. The results show that the agents are able to learn and achieve asymptotic performance faster in the target task when the fuzzy rule transfer is applied. To the best of our knowledge, this is the first work to investigate the possibility of using transfer learning in the fuzzy reinforcement learning domain.

\subsection{Contributions}

The main contributions of this thesis are:

1. We investigate the possibility of using transfer learning in the fuzzy reinforcement learning domain.

2. We learn from the concept of transfer learning methods in the literature [7], particularly state value transfer [8] and policy transfer [9]. Building on these techniques, we propose a novel approach to target knowledge transfer among tasks using FACL algorithm.

3. We designed a fuzzy rule transfer method (FRT) to promote knowledge transfer between the source and target task by mapping the fuzzy rules between the FACL agents.

4. We evaluate our method in the differential game domain where the state space and the action space are continuous. 


\subsection{Thesis Organization}

The thesis is organized as follows:

- Chapter 2 presents background information on reinforcement learning and fuzzy reinforcement learning. We provide an overview on key concepts in reinforcement learning including Markov decision process (MDP), state value and action value, and temporal-difference learning. We present an overview on the fuzzy system and the fuzzy actor-critic learning algorithm later in the chapter.

- Chapter 3 introduces background information on transfer learning in reinforcement learning domain, particularly the main concepts in transfer learning. We have also showed an elaborated transfer learning example of inter-task mapping in the Paddle Game in this chapter.

- Chapter 4 presents our novel approach for transfer learning in the FACL algorithm. Full details of fuzzy rule transfer (FRT) method and key concepts such as similarity in the fuzzy rules are presented in this chapter. We evaluate our method in the two sets of differential game; the pursuit-evasion game and the guarding-a-territory game. Further details are provided on the design of the differential game and the application in the FACL agents. We present the simulation results that demonstrate the performance of our transfer methods in the differential games.

- Chapter 5 presents the conclusion of the thesis and potential future directions. 


\section{Chapter 2}

\section{Reinforcement Learning and Fuzzy Reinforcement Learning}

Learning is a natural process that occurs throughout our lifespan, particularity when we discover new ideas, knowledge and skills. Many types of learning can be seen as a result of interaction between the environment and ourselves. This interaction provides causality and consequences of actions and such interaction are the primary source for gaining our knowledge. Interacting with environment is also the central idea in reinforcement learning.

Reinforcement learning is different from supervised learning. In supervised learning, the agent learns from a training set of labeled data provided by a knowledgeable external supervisor [1]. The goal of supervised learning is to generalize a response or prediction such that it acts correctly in situations which is not present in the data from history. One of the biggest problems in supervised learning is that it is often impractical for the agent to obtain samples of desired actions that are both correct and representative of all the situations, particularly in the situation where the training data is not available or it is impossible to generate data beforehand. Reinforcement

learning is designed to solve these types of problems by letting the agent learn from 
its own experience. The learning process is guided by the reward generated throughout the interaction with the environment. Therefore, the agent needs to explore its available actions to evaluate which actions yield the most reward. The agent should also exploit its action in a greedy way in order to improve its performance, known as maximizing its reward, in the long run. This is one of the challenges in reinforcement learning; the trade-off between exploration and exploitation [1].

Fig. 2.1 illustrates the agent-environment interaction. Suppose the agent interacts with the environment in discrete time steps $t=0,1,2, \cdots$. At each time step $t$, the agent is marked with a state $s_{t} \in S$ from the environment, where $S$ is the state space. Meanwhile, the agent also executes an action $a_{t} \in A$ based on the current state $s_{t}$, where $A$ is the action space. At the next time step $t+1$, as the consequence of the action $a_{t}$, the environment transits into a new state $s_{t+1}$ and a numerical reward signal is generated and received by the agent [2]. The reward indicates the performance of action $a_{t}$ in state $s_{t}$. The agent has to retain a memory of the actions producing the most reward from previously encountered situations, and use this memory to choose the action from its action space in the future based on the future state.

The mapping from states to probabilities of selecting each possible action is called the agent's policy [1]. We can denote the agent's policy as $\pi_{t}$, and $\pi_{t}(s, a)$ is the probability of choosing $a_{t}=a$ from the action space at state $s_{t}=s$. During the course of reinforcement learning, the agent updates its policy based on its experience interacting with the environment to maximize the total amount of reward it receives over the long run. We will cover these aspects in the following sections in this chapter.

This chapter provides background information on reinforcement learning. We will discuss many important concepts and methods ranging from the fundamental Markov decision processes (MDP) framework to the widely used actor-critic learning methods. The chapter is organized as follows: 


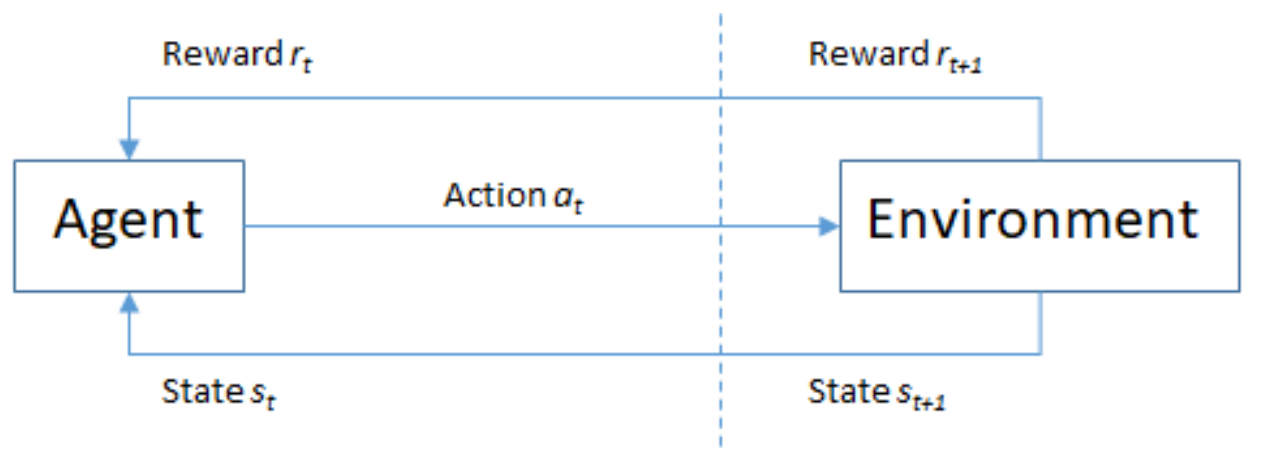

Figure 2.1: The agent-environment interaction in reinforcement learning

- Section 2.1 introduces the Markov Decision Process (MDP)

- Section 2.2 shows the concept of reward and return

- Section 2.3 presents the value function and policy

- Section 2.4 describes the temporal difference learning method

- Section 2.5 presents the fuzzy rules and fuzzy systems

- Section 2.6 introduces the fuzzy actor critic learning algorithm

\subsection{Markov Decision Process}

We will introduce the finite Markov decision processes, or finite MDPs in this section. MDPs are considered as a classical formalization of sequential decision making. The actions in MDPs do not just impact the immediate rewards, but also the subsequent situations, or states, and through those future rewards [1]. Since the states are dependent on the MDPs environment, we need to take into account the delayed reward and the trade off between the short-term reward and long-term reward. As we mentioned earlier, the agent needs to explore all its available actions to evaluate which actions yield the most reward. Meanwhile the agent also needs to exploit its 
current best action in a greedy way in order to maximize its reward. However, since the maximal long-term reward is not foreseeable without exploration and discovery, the balance between exploration and exploitation needs to be carefully determined.

The learner interacting with the environment is called agent while the environment comprises everything outside the agent. We have already presented this interaction in Fig. 2.1. The MDP is called a finite MDP if the state and action spaces are finite [1]. The dynamics of a finite MDP can be efficiently described as [1]

$$
p\left(s^{\prime}, r \mid s, a\right):=\operatorname{Pr}\left\{S_{t+1}=s^{\prime}, R_{t+1}=r \mid S_{t}=s, A_{t}=a\right\}
$$

where $S_{t} \in S$ and $A_{t} \in A(s)$ and $\operatorname{Pr}\left\{S_{t+1}=s^{\prime}, R_{t+1}=r \mid S_{t}=s, A_{t}=a\right\}$ indicates the transitioning probability of getting to state $s^{\prime}$ with the reward $r$ after choosing action $A_{t}=a$ in state $S_{t}=s$. We follow the notation used in the Sutton's book [1], where capital letters are used for random variables and lowercase letters are used for the values of random variables. We can clearly observe that in MDPs, the next state $S_{t+1}$ and $R_{t+1}$ depend only on the current state $S_{t}$ and action $A_{t}$ and not on the history of previous successive states and actions leading to $S_{t}$. For example, the state and action prior to time $t\left(S_{t-1}, A_{t-1}, S_{t-2}, A_{t-2}, \ldots e t c\right)$ is not relevant . Namely, the current state $S_{t}$ has all the information necessary to decide the next action $A_{t}[1]$. The state-transition probabilities is a three argument function, defined as

$$
p\left(s^{\prime} \mid a, s\right)=\operatorname{Pr}\left\{S_{t+1}=s^{\prime} \mid S_{t}=s, A_{t}=a\right\}
$$

The transition function is a probability distribution, it satisfies

$$
\sum_{s^{\prime}} p\left(s^{\prime} \mid a, s\right)=1 \quad \forall s \in S, a \in A
$$




\subsection{Reward and Return}

In reinforcement learning, the purpose of the agent is to solve a particular task, however this solving process is guided by a special reward signal, passing from the environment to the agent. The reward is in the form of a numerical number and the goal of the agent is to maximize the total reward it receives during the course of learning, including both the present and future reward $R_{t}$. This means if the sequence of the rewards received after time step $\mathrm{t}$ is denoted as $r_{t+1}, r_{t+2}, r_{t+3}, \ldots$, then the total reward an agent seeks to maximize is the expected return $R_{t}$, defined as

$$
R_{t}:=r_{t+1}+r_{t+2}+r_{t+3}+\cdots+r_{T}
$$

Where $\mathrm{T}$ is the terminal time. Reinforcement learning tasks with an associated terminal state is called an episodic task [1]. However, not all reinforcement learning tasks can be defined within an episode and have a terminal state. Some tasks continue indefinitely. Reinforcement learning tasks that continue indefinitely are referred to as continuous tasks [1]. Calculating the return in Eq.(2.4) is problematic for continuing tasks because the final time step would approach infinity, and the total expected reward would also be infinite. The cumulative reward $R_{t}$ for a continuous task has an infinite value and is given as

$$
R_{t}:=r_{t+1}+r_{t+2}+r_{t+3}+\cdots=\sum_{k=0}^{\infty} r_{t+k+1}
$$

One approach to solve this problem is to introduce the concept discounting. The revised expected return is shown in

$$
R_{t}=r_{t+1}+\gamma r_{t+2}+\gamma^{2} r_{t+3}+\cdots=\sum_{k=0}^{\infty} \gamma^{k} r_{t+k+1}
$$


In this equation, an additional discounting factor $\gamma(0 \leq \gamma \leq 1)$ is introduced to the reward function. The discount factor is increased exponentially according to the time step of the reward.

\subsection{Value Function and Policy}

The state-value function indicates the value of any given state in a long run [1]. It defines how good a particular state is in the MDP framework of reinforcement learning. The agent has to take the state-value function into more consideration in deciding future actions in order to perform well in the long-term. We have already discussed the return function in the previous section. It can be used to provide the agent with information of the expected performance of a given action $a_{t}$ in state $s_{t}$. The agent computes a state-value function for every state $s \in S$ based on the expected return.

In reinforcement learning, a policy is a mapping from states to probabilities of selecting each action $a \in A_{t}$. For instance, $\pi(a \mid s)$ means the probability that $A_{t}=a$ when $S_{t}=s$ provided agent is following a policy $\pi$ at time $t$. We define the state-value 
function $V^{\pi}(s)$ following the policy $\pi$ as follow [1]

$$
\begin{aligned}
V^{\pi}(s) & =E_{\pi}\left\{R_{t} \mid S_{t}=s\right\} \\
& =E_{\pi}\left\{\sum_{k=0}^{\infty} \gamma^{k} r_{t+k+1} \mid s_{t}=s\right\} \\
& =E_{\pi}\left\{r_{t+1}+\gamma \sum_{k=0}^{\infty} \gamma^{k} r_{t+k+2} \mid s_{t}=s\right\} \\
& =E_{\pi}\left\{r_{t+1}+\gamma V^{\pi}\left(s^{\prime}\right) \mid s_{t}=s\right\} \\
& =\sum_{a} \pi(s \mid a) \sum_{s^{\prime}} p\left(s^{\prime} \mid a, s\right)\left\{R\left(s^{\prime} \mid a, s\right)+\gamma V^{\pi}\left(s^{\prime}\right)\right\}
\end{aligned}
$$

where the $s^{\prime}$ is next state, $p\left(s^{\prime} \mid a, s\right)$ is the state-transition probability, $\pi(s \mid a)$ is the probability assigned for taking action $a$ in state $s$ under policy $\pi$ and $E_{\pi}\{\}$ denotes the expected value given that the agent acts under the policy $\pi$. The policy $\pi$ is a probability distribution and it satisfies

$$
\sum_{a} \pi(x \mid a)=1 \quad \forall x \in X, a \in A
$$

Similarly, we define the action-value function $Q^{\pi}(s, a)$ acting under the policy $\pi$ as the expected return starting from state $x$, selecting action $a$ and thereafter following policy $\pi$ given as [1]

$$
\begin{aligned}
Q^{\pi}(s, a) & =E_{\pi}\left\{R_{t} \mid S_{t}=s, A_{t}=a\right\} \\
& =E_{\pi}\left\{r_{t+1}+\gamma V^{\pi}(s) \mid S_{t}=s, A_{t}=a\right\} \\
& =\sum_{s^{\prime}} p\left(s^{\prime} \mid a, s\right)\left\{R\left(s^{\prime} \mid a, s\right)+\gamma V^{\pi}\left(s^{\prime}\right)\right\}
\end{aligned}
$$

Equation Eq.(2.7) is called the Bellman equation for the state-value function $V^{\pi}(x)$. 
It represents a relationship between the value of a state and the value of its successor state. The Bellman equation averages over all the possibilities, weighting each by its probability of occurring [1]. Equation Eq.(2.9) is the action-value function $Q^{\pi}(x, a)$ acting under policy $\pi$ [1]. The action-value function $Q(x, a)$ determines the value of each state-action pair also known as the Q-values. If the agent always chooses the actions with the highest estimated Q-values, the agent is said to be following the greedy strategy [2].

\subsection{Temporal-Difference Learning}

The TD method is used by agents to learn from raw experience without knowing the underlying model or dynamics of the environment. The TD method also performs bootstrapping, which means it updates previous estimates on the basis of new estimates. The estimated result will propagate from state to state during the course of the computation [1]. The TD based method is one of the most important concepts in the reinforcement learning world. We will briefly cover the TD prediction, SARSA and Q-learning in this section.

\subsubsection{TD Prediction}

We refer policy evaluation such as estimating the value function $V_{\pi}$ for a given policy $\pi$ as the prediction problem. In the TD prediction problem, the TD method only needs to wait until the next time step to make the update for the state value function, shown as [1]

$$
V\left(S_{t}\right)=V\left(S_{t}\right)+\alpha\left[R_{t+1}+\gamma V\left(S_{t+1}\right)-V\left(S_{t}\right)\right]
$$


Where $\alpha$ is the learning rate and $\gamma$ is the discount factor . The difference quantity in brackets in Eq.(2.10) is used to compute an error signal $\Delta$. This error signal $\Delta$ is called the TD error and is given as [1]

$$
\Delta=R_{t+1}+\gamma V\left(S_{t+1}\right)-V\left(S_{t}\right)
$$

The update of state value happens immediately on transition to the next state $S_{t+1}$ and receiving reward $R_{t+1}$. This simple TD method is called TD(0) or one step TD. We show a complete version of $\operatorname{TD}(0)$ algorithm in Algorithm 2.1 [1].

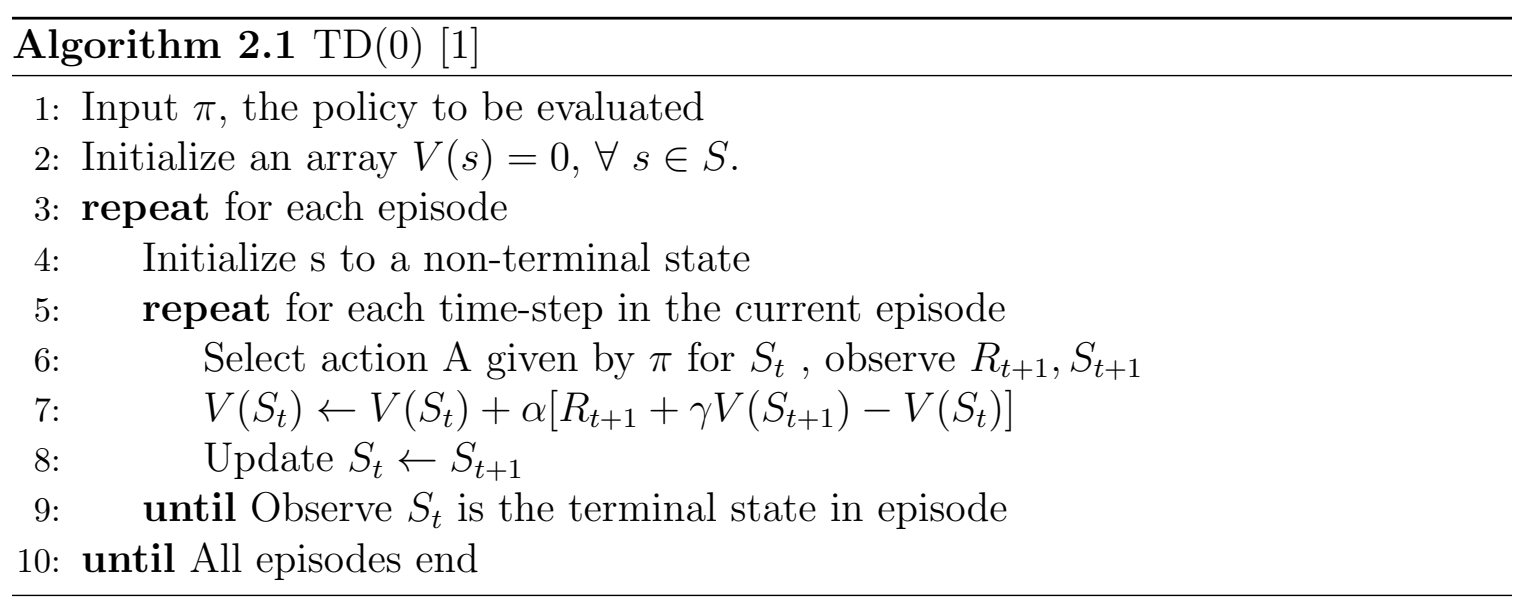

\subsubsection{Q-learning}

Q-learning was one of the early breakthroughs in reinforcement learning and it was first introduced by Watkins [10]. Q-learning is a TD based learning method that recursively finds a solution to the Bellman equation given in Eq.(2.9). Similar to SARSA learning, Q-learning is useful when the state transition probabilities and the complete knowledge of the underlying dynamics of the environment is unavailable. Q-learning uses each new experience of the agent to immediately approximates the optimal action-value function $q_{*}$. This is an off-policy approach which means the 
policy being evaluated is not necessarily the same as the behavior policy. The update rule of Q-learning is defined as

$$
Q\left(S_{t}, A_{t}\right)=Q\left(S_{t}, A_{t}\right)+\alpha\left[R_{t+1}+\gamma \max _{a^{\prime}} Q\left(S_{t+1}, A_{t+1}\right)-Q\left(S_{t}, A_{t}\right)\right]
$$

for any finite MDP, the Q-learning algorithm is proved to converge to the optimum Q-values when the state-action pairs are visited infinite times and the learning rate is adequately decreased over time [11]. As for the exploration-exploitation in action selection, the common strategy is using $\epsilon$-greedy action selection policy, meaning that for most of the time the action would generate maximal estimated action value [2]. The $\epsilon$-greedy policy chooses a greedy action $a_{*} \in A$ with probability $\epsilon$ or the random action $a_{r} \in A$ with probability $1-\epsilon$ given as [2]

$$
A_{t}= \begin{cases}a_{*} & \text { with prob }(\epsilon) \\ a_{r} & \text { with prob }(1-\epsilon)\end{cases}
$$

We present the Q-learning algorithm in Algorithm 2.2 as follows [1].

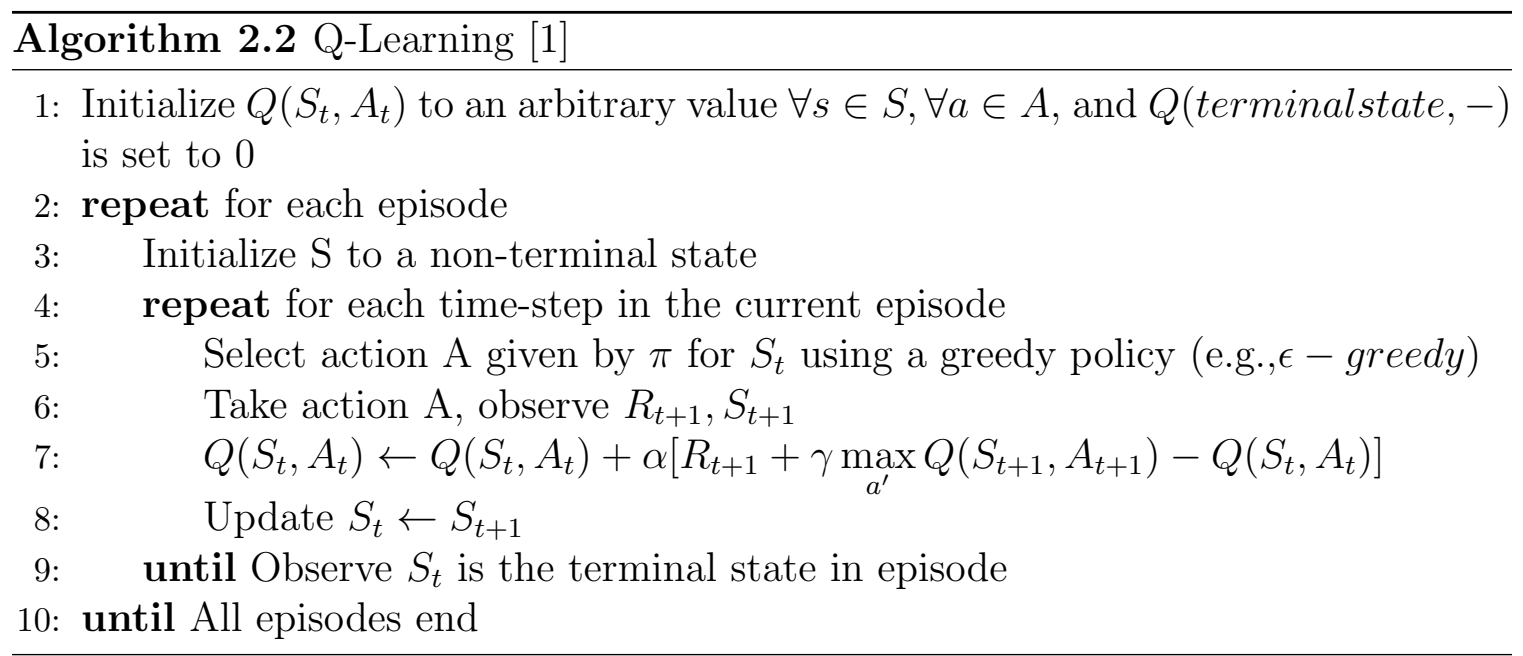

However, Q-Learning has many drawbacks. A major limitation of Q-learning is 
that it only works in environments with discrete and finite state and action spaces. One solution for extending Q-learning to richer environments is to apply function approximators to learn the value function, taking states as inputs, instead of storing the full state-action table (which is often infeasible). In the reinforcement learning community this is typically a linear function approximator, but sometimes a nonlinear function approximator is used instead, such as a neural network. We refer to a neural network function approximator as a Q-network [12]. In Section 3.4, we will show a transfer learning example in the paddle game where the agent is learning using the Q-network.

\subsection{Fuzzy Rules and Fuzzy systems}

Fuzzy systems have been applied to a wide variety of fields ranging from control, signal processing, communications, integrated circuit manufacturing, and expert systems to business, medicine, psychology, etc. However, the most significant applications have concentrated on control problems [13]. Fuzzy systems are knowledge-based or rulebased systems and the heart of the fuzzy system is the fuzzy IF-THEN rule. A fuzzy IF-THEN rule is an IF-THEN statement in which some words are characterized by continuous membership functions [13]. An example of the fuzzy rule can be:

If the speed of a car is slow, Then apply more force to the gas

where the words "slow" and "more" are characterized by the membership functions for the linguistic variable speed of the car and the force to the gas. A fuzzy system is constructed from a collection of fuzzy IF-THEN rules [13]. There are three types of fuzzy systems that are commonly used in the literature: (i) pure fuzzy systems, (ii) Takagi-Sugeno-Kang (TSK) fuzzy systems, and (iii) fuzzy systems with fuzzifier and 


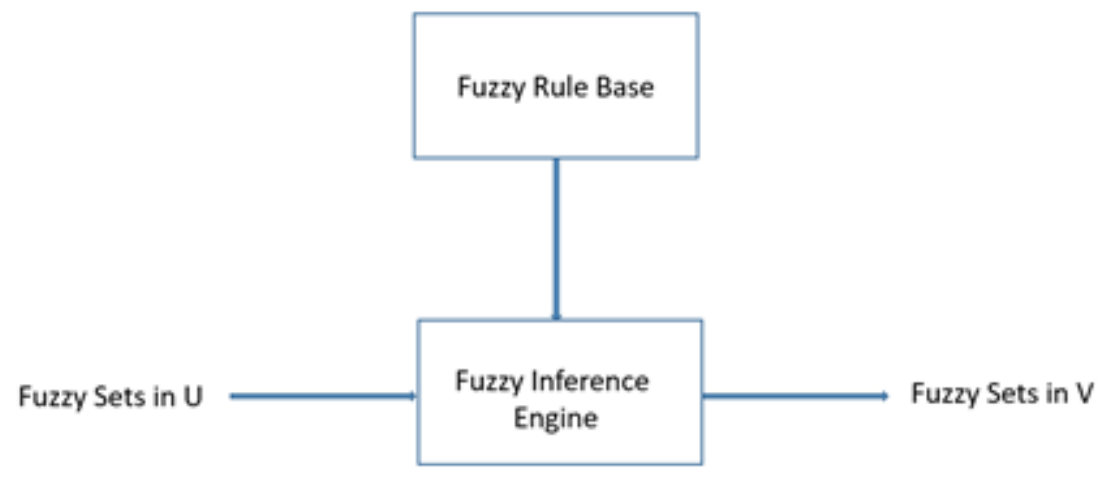

Figure 2.2: Basic configuration of pure fuzzy systems

defuzzifier [13]. We briefly describe these three types of fuzzy systems as follows.

- In a basic pure fuzzy systems, the fuzzy inference engine combines the fuzzy IF-THEN rules into a mapping from fuzzy sets the input space $U \subset R^{n}$ to fuzzy sets in the output space $V \subset R$ based on fuzzy logic principles [13]. We show an example of the basic configuration of pure fuzzy systems in Fig. 2.2. The main drawback with the pure fuzzy system is that its inputs and outputs are fuzzy sets, whereas in engineering systems the inputs and outputs are real-valued variables.

- In Takagi-Sugeno-Kang (TSK) fuzzy systems, the above problem is solved with a different fuzzy system design where the inputs and outputs are real-valued variables [13]. The Takagi- Sugeno-Kang (TSK) system uses rules in the following form:

If the speed $x$ of a car is slow, Then the force applied to the throttle is $y=a x$

where $\mathrm{x}$ is the linguistic variable describing the speed of a car, $\mathrm{y}$ is the output 


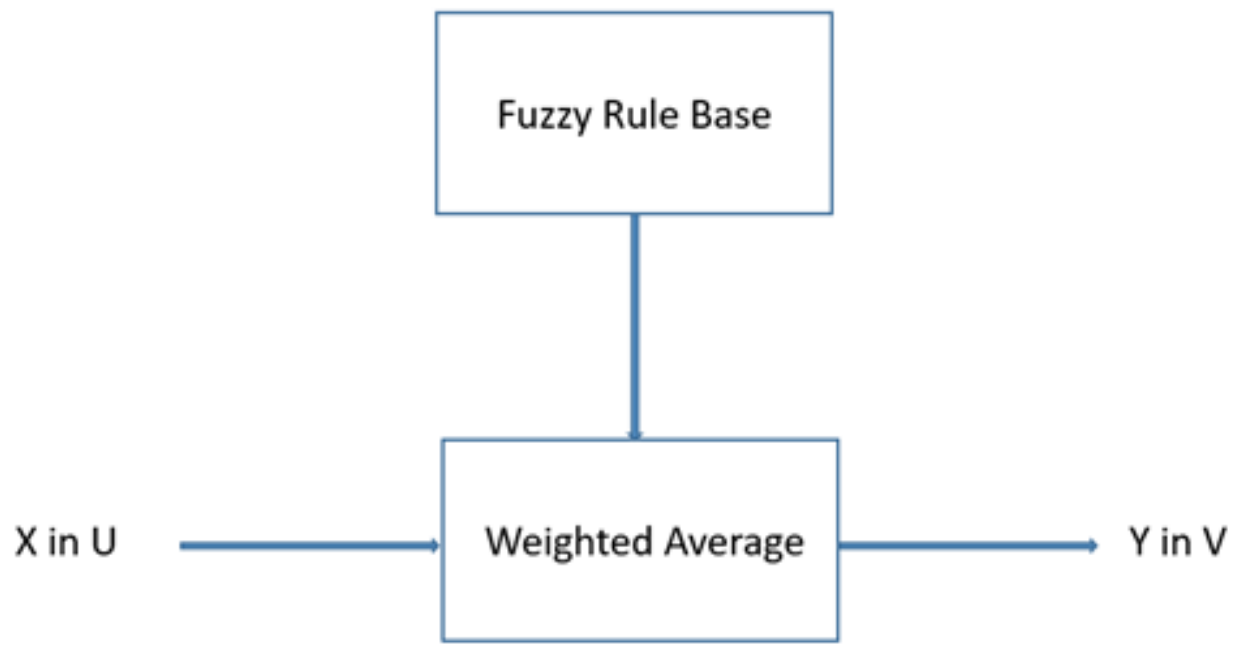

Figure 2.3: Basic configuration of Takagi-Sugeno-Kang fuzzy system

to generate force and a is a constant. The basic configuration of Takagi-SugenoKang fuzzy system is presented in Fig. 2.3. There are two problem associated with TSK fuzzy system [13]: (i) its THEN part is a mathematical formula and therefore may not provide a natural framework to represent human knowledge, and (ii) there is not much freedom to apply multiple fuzzy sets as the input to the system so that the versatility of fuzzy systems is not well-represented in this framework.

- A Fuzzifier and defuzzifier is used to solve the above problems in TSK fuzzy system. Fuzzy systems with a fuzzifier and a defuzzifier are able to transform a real-valued variable into a fuzzy set as the input, and use defuzzifier to transform a fuzzy set into a real-valued variable as output [13]. We show the resulting fuzzy system in Fig. 2.4. This fuzzy system overcomes the disadvantages of the pure fuzzy systems and the TSK fuzzy systems [13]. 


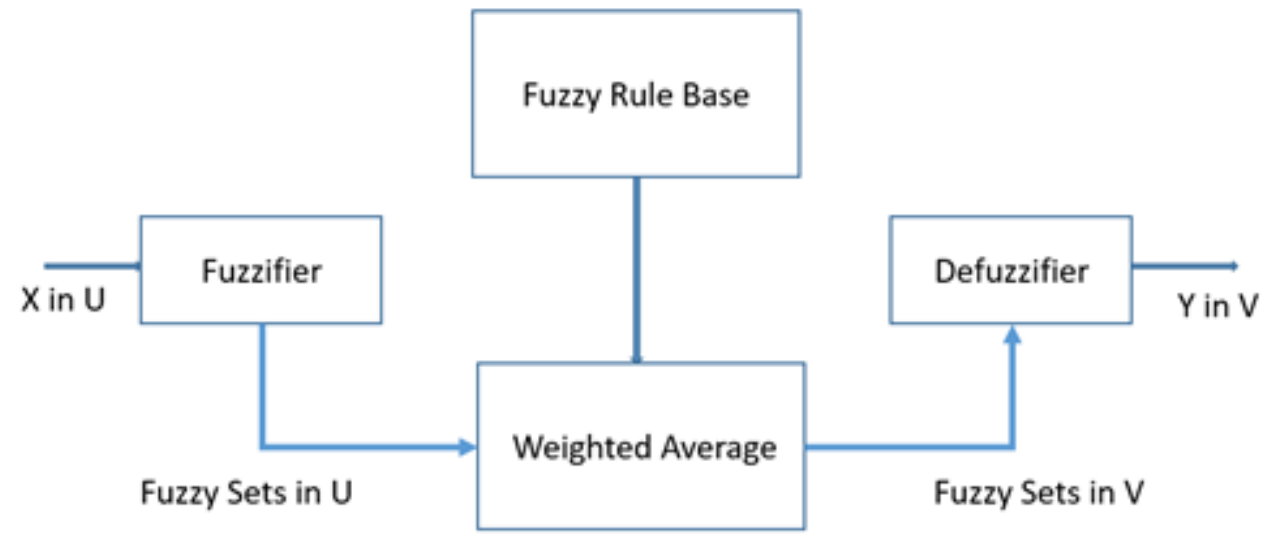

Figure 2.4: Basic configuration of fuzzy systems with fuzzifier and defuzzifier.

\subsection{Fuzzy Actor Critic Learning}

When the state and action spaces are large or in the continuous domain, it is usually intractable to maintain a look-up table. As a result, traditional reinforcement learning algorithms such as Q-learning may lead to the curse of dimensionality. Function approximators such as a cerebellar model arithmetic computer (CMAC) or an artificial neural network (ANN) can be used to represent the continuous state and action spaces in an effective way [1]. One of the widely used function approximators is the fuzzy logic controller (FLC) or fuzzy inference systems (FIS) [2]. Fuzzy Inference Systems (FIS) are popular computing frameworks based on the concepts of fuzzy set theory, which have been extensively applied with success in many fields like

control, decision support, system identification etc [14]. When reinforcement learning is associated with the FLC, the approach is commonly called fuzzy reinforcement learning $[5]$.

In the fuzzy actor-critic learning algorithm, the fuzzy inference system(FIS) is implemented as a function approximator for both actor and critic. The role of the 
actor is to generate the continuous control action while the role of the critic is to predict the sum of future discounted reward. Fig. 2.5 illustrates the FACL agent and its environment to the system block. In our previous research, we have applied the fuzzy reinforcement learning methods to the pursuer-evader differential game in [15], [16]. In [15], a fuzzy actor-critic learning (FACL) algorithm is also applied to the pursuit-evasion differential game. It is shown that the adaptive fuzzy critic in [16] performed better than the neural network proposed in [17]. In the configuration proposed in this thesis, we only adapt the output parameters of the fuzzy system, whereas in [16], [18] the input and output parameters of the fuzzy system are adapted which is a more complex adaptive algorithm.

In the previous sections we have covered a few important concepts in reinforcement learning. A reinforcement learning agent interacts with the MDP environment and receives a reward signal $r_{t}$ at each time step. The ultimate goal of an agent is to maximize the discounted return $R_{t}$ in a long run [1], as shown in Equation(2.6). The value function is the expected rewards at time step $t$, and can also be rewritten recursively as

$$
V_{t}=r_{t+1}+\gamma V_{t+1} .
$$

The agent immediately receives a reward from the current environment based on its action in that state. We use a FIS as agent's critic to estimate the value of the state at time $t$, as $\hat{V}(s(t))$. The error in the estimation of the state value is represented by the temporal difference. As shown in Fig. 2.5, the temporal difference (TD) error $\delta_{t}$, is given as

$$
\delta_{t}=r_{t+1}+\gamma \hat{V}\left(s_{t+1}\right)-\hat{V}\left(s_{t}\right)
$$

We represent the actor by an adaptive fuzzy controller to effectively select an 


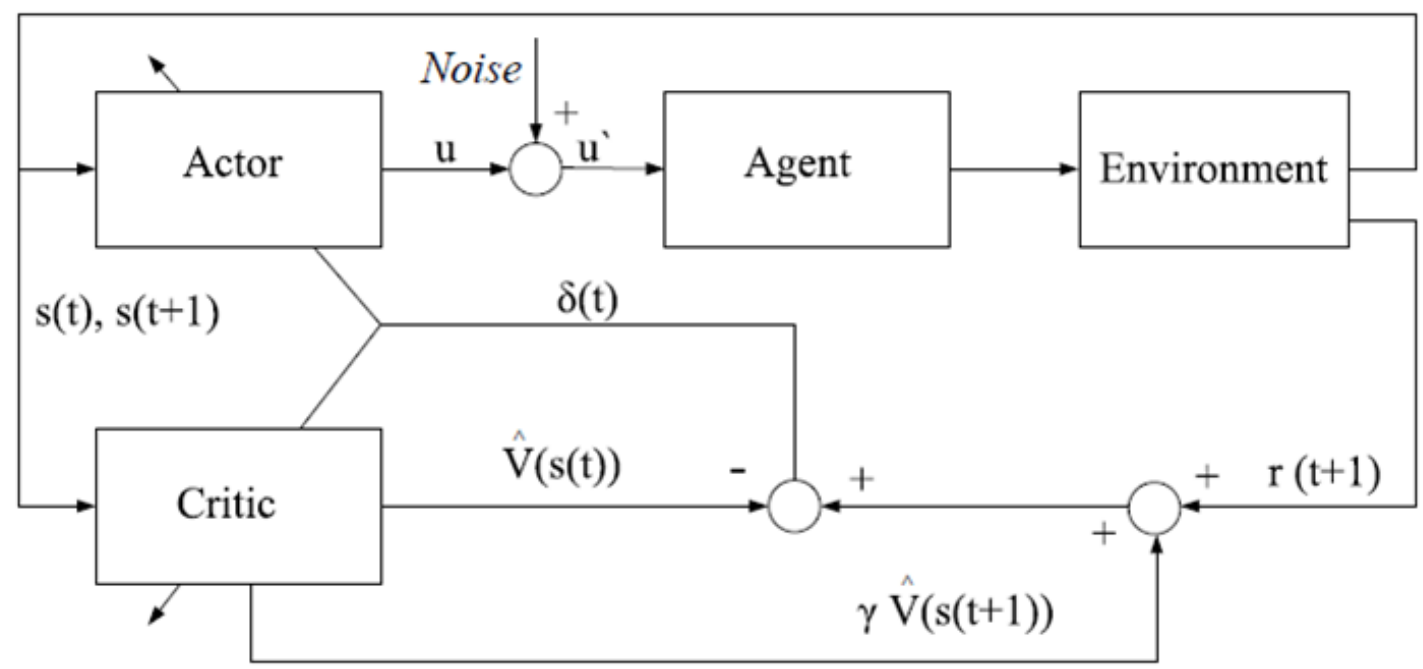

Figure 2.5: The fuzzy actor critic learning structure.

Source: [19]

action and also update the policy parameter in the FLC in the direction suggested by the Critic. The output of the fuzzy controller is,

$$
u_{t}=\sum_{l=1}^{M} \Phi^{l} w_{t}^{l}
$$

where $M$ is the number of rules, $w^{l}$ is the output or consequent parameter of the actor, and $\Phi^{l}$ is the firing strength of rule $l$. The firing strength for rule $l$ is defined as,

$$
\Phi^{l}=\frac{\prod_{i=1}^{n} \mu^{F^{l}}\left(x_{i}\right)}{\sum_{l=1}^{M}\left(\prod_{i=1}^{n} \mu^{F_{i}^{l}}\left(x_{i}\right)\right)}
$$

where $n$ is the number of inputs and $\mu^{F_{i}^{l}}$ is the membership degree of input $x_{i}$, in the fuzzy rule $F_{i}^{l}$. We choose only triangular membership functions where only two membership functions overlap for any given input and the maximum value of any 


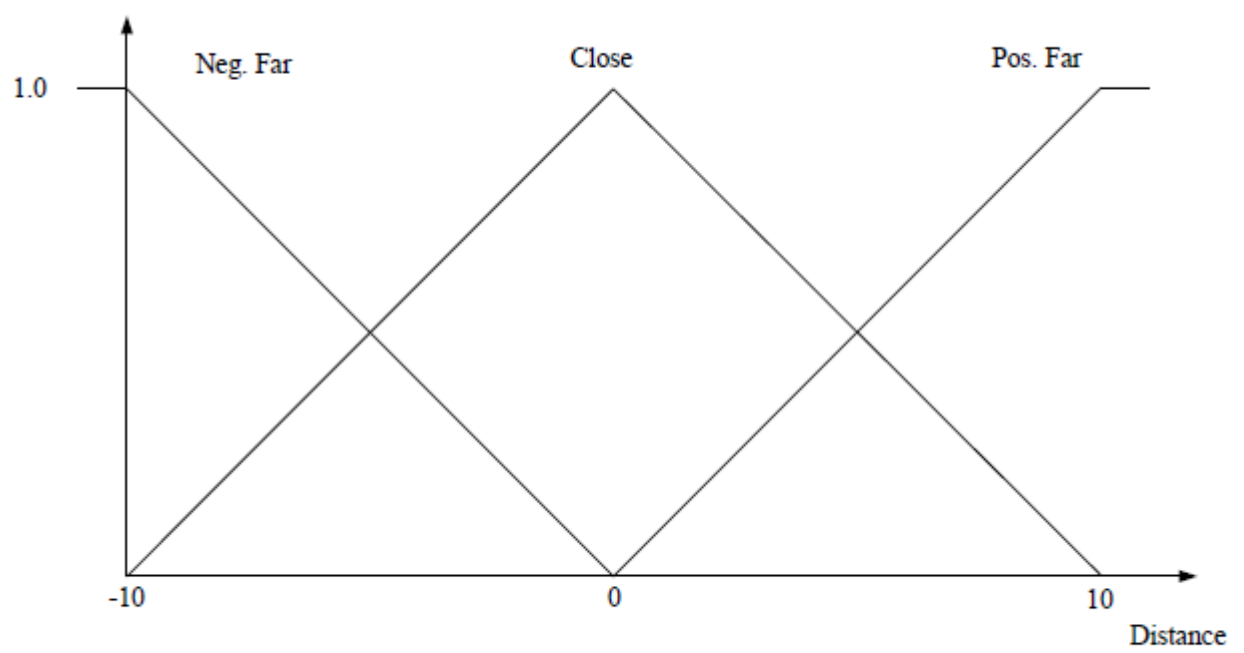

Figure 2.6: Membership function for input of distance.

Source: [19]

membership function is 1 [19]. Therefore the denominator of (2.17) is always equal to 1. For example, the set of membership functions for distance are as shown in Fig. 2.6. We can then rewrite (2.17) as [19],

$$
\Phi^{l}=\prod_{i=1}^{n} \mu^{F_{i}^{l}}\left(x_{i}\right) .
$$

In order to promote exploration of the action space, a random white noise is chosen from a Gaussian distribution with a mean of 0 and a variance of $\sigma$ given by $N(0, \sigma)$ is added to the generated control signal $u$. The output parameter of the actor FLC, $w^{l}$ is adapted as [19]

$$
w_{t+1}^{l}=w_{t}^{l}+\beta_{L} \delta_{t}\left(u_{t}^{\prime}-u_{t}\right) \frac{\partial u}{\partial w^{l}}
$$

where $\delta_{t}$ is the TD error mentioned earlier, $\beta_{L} \in(0,1)$ is the learning rate for the actor and where,

$$
\frac{\partial u}{\partial w^{l}}=\Phi_{t}^{l} .
$$


Upon examining (2.19) we see that the term $u_{t}^{\prime}-u_{t}$ is equal to the added noise. As such we modify the update for the actor FLC output parameters as,

$$
w_{t+1}^{l}=w_{t}^{l}+\beta_{L} \delta_{t}(\text { noise }) \Phi_{t}^{l}
$$

Once the action is executed, the critic evaluates the new state to determine the expected new state value. The output of the critic $\hat{V}$ is an approximation to $V$ given by

$$
\hat{V}_{t}=\sum_{l=1}^{M} \Phi^{l} \zeta_{t}^{l}
$$

$\zeta_{t}^{l}$ is the output or consequent parameter of the critic and $\Phi^{l}$ is defined in (2.17). The critic output parameter $\zeta^{l}$ is adapted as,

$$
\zeta_{t+1}^{l}=\zeta_{t}^{l}+\alpha_{L} \delta_{t} \frac{\partial \hat{V}}{\partial \zeta^{l}}
$$

where $\alpha_{L} \in(0,1)$ is the learning rate for the critic. The learning rate in FACL is set as $\beta_{L}<\alpha_{L}$, so that the actor will converge slower than the critic to prevent instability in the actor [20]. The partial derivative is calculated as,

$$
\frac{\partial \hat{V}}{\partial \zeta^{l}}=\Phi_{t}^{l} .
$$

We update the parameters in critic as

$$
\zeta_{t+1}^{l}=\zeta_{t}^{l}+\alpha_{L} \delta_{t} \Phi^{l}
$$

The FACL learning algorithm is presented in Algorithm 2.3. 


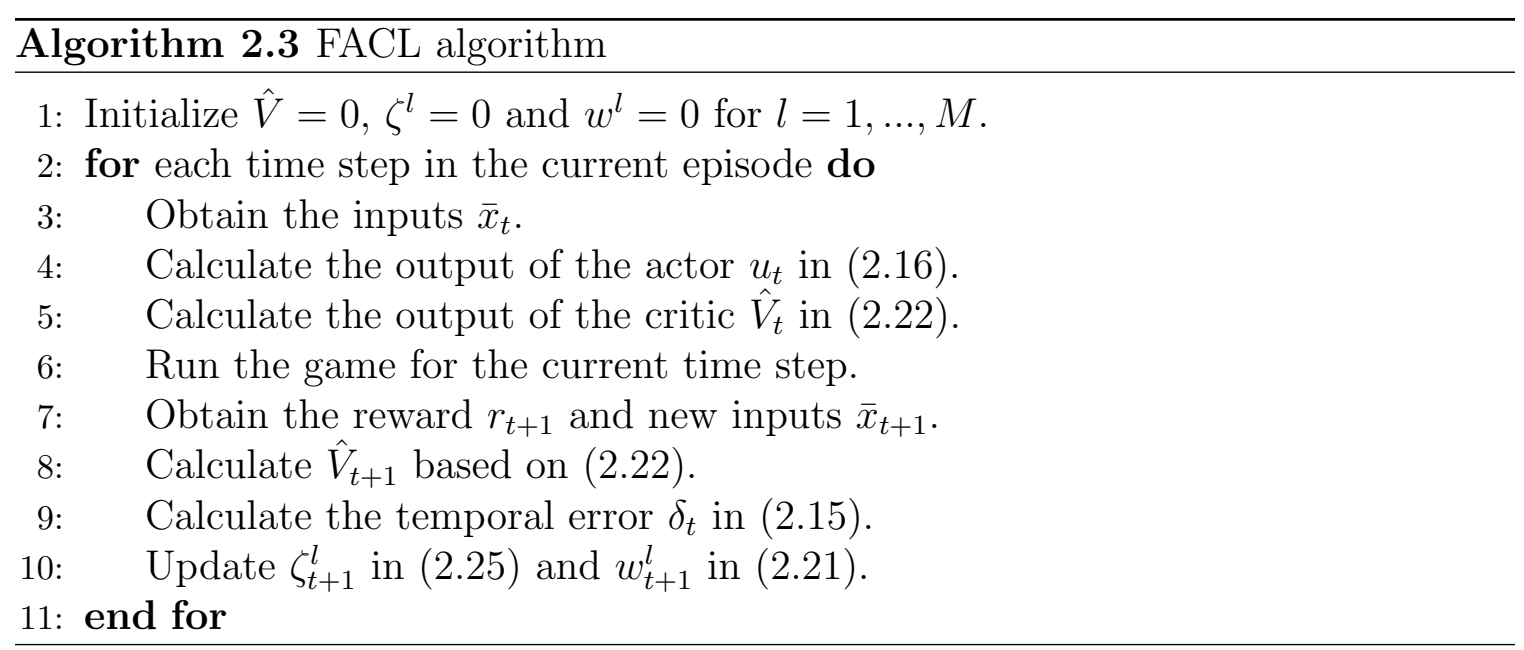

\subsection{Summary}

In this chapter, we presented a brief introduction to the relevant reinforcement learning concept. We introduced the concept of Markov decision process. We showed the concept of reward and return, the notion of value function and policy. The temporal difference learning method is also briefly described. In the second part of the chapter we provided an overview on fuzzy rules and system. The concepts and algorithms presented in this chapter are essential to our research and will be used in Chapter 4. 


\section{Chapter 3}

\section{Transfer Learning}

Reinforcement learning is gaining significant attention in the field of artificial intelligent. One of the biggest challenges in this area is the slow convergence speed to a good policy when agents try to master complex tasks . For instance, temporaldifference(TD) learning [1] is a type of reinforcement learning that has shown some success in different machine learning tasks. TD based methods have the ability to learn when there is limited prior knowledge and minimal environmental feedback [6]. However, TD can be slow in practice to produce near-optimal behaviors. Many techniques exist which attempt, with more or less success, to speed up the learning process [6]. Thus, a large amount of the research targets at improving the learning speed of agents, which includes using transfer learning in RL. The insight behind transfer learning is that generalization may occur not only within tasks, but also across tasks [7]. The idea is similar to the human learning process; we use our previous experience to efficiently tackle new tasks. Similarly, the way of learning can also benefit the RL agents given the appropriate experience from a similar task they have learned previously. The most intuitive way to apply TL to RL is to reuse the solution of previous tasks that have already been presented to the agent [8], but many methods also focus on reusing knowledge from external sources, such as demonstrations 
from humans or advice from other learning agents [21]. In the reinforcement learning context, we aim to transfer knowledge acquired by agents in a previous task (source task) to a new task (target task) in order to speed up learning.

There have been many studies in transfer learning for RL tasks (e.g., [22]), [23]). The majority of the transfer learning algorithms vary depending on the level of similarity between the target task and source task [7]. The allowed task differences are a major consideration in RL transfer learning methods. To mimic a real world situation, we prefer methods that are flexible to allow state variables to differ between the source and target task. One of the effective approaches is transfer via inter-task mapping (TVITM) which enables transfer between pairs of tasks by mapping the state variables and actions in the target task to the source task [8]. In their research, different function approximators are successfully used including the artificial neural network (ANN) and cerebellar model articulation controller (CMAC). Another good approach fitting into the scenario is transfer via inter-task mapping for policy search methods (TVITM-PS) [9]. In this approach, instead of transferring the action-value functions in TVITM, the entire policies are transferred between the source and target tasks. Again, the transfer in TVITM-PS is performed and evaluated on a neural network function approximator. We will briefly cover these two methods - TVITM and TVITM-PS here.

This chapter provides background information on transfer learning. The chapter is organized as follows:

- Section 3.1 provides a brief introduction on transfer learning

- Section 3.2 shows transfer learning via inter-task mappings

- Section 3.3 presents transfer learning via inter-task mappings in policy search 
- Section 3.4 provides an example of applying transfer learning to the paddle game

\subsection{Transfer Learning Overview}

Why is transfer learning an important topic to address in reinforcement learning ? There are three reasons mentioned in the literature, these are [7]:

1. First, RL techniques have made tremendous progress and have achieved notable successes in recent years. They are now able to handle difficult tasks which other machine learning techniques are either unable to address or perform poorly. One of the well-known examples is Alpha Go [24], which is able to learn and achieve superhuman performance in the game of Go.

2. Second, classical machine learning techniques such as rule induction and classification are sufficiently mature that they may now easily be leveraged to assist with TL.

3. Third, there have been many results of the possibility of using transfer learning. Moreover, many of those transfer learning method can be very effective at speeding up learning. For example, "Autonomous Transfer for Reinforcement Learning" can automatically learn a mapping from one task to another through an agent's experience.

Many questions arise when we investigate transfer learning methods. The following questions distinguish transfer methods and are discussed in the literature [7]:

1. What are the goals and metrics used in transfer learning? We will cover the most commonly used metrics in this chapter. 
2. What type of information is transferred between tasks? The kind of information can range from very low-level information (the knowledge of direct control of the agent) to high-level information (information such as how a particular domain functions)

3. What information is transferable and how does a transfer method make decisions based on this information?

4. How similarity is assumed between tasks? The source and target task can vary in many ways. (e.g. state-space differences and action-space differences)

We will cover these questions as we briefly go through a few important concepts in this section. First, different ways of evaluating transfer learning methods will be discussed. Then, we will cover the five dimensions of transfer learning.

\subsubsection{Evaluating Transfer Learning Methods}
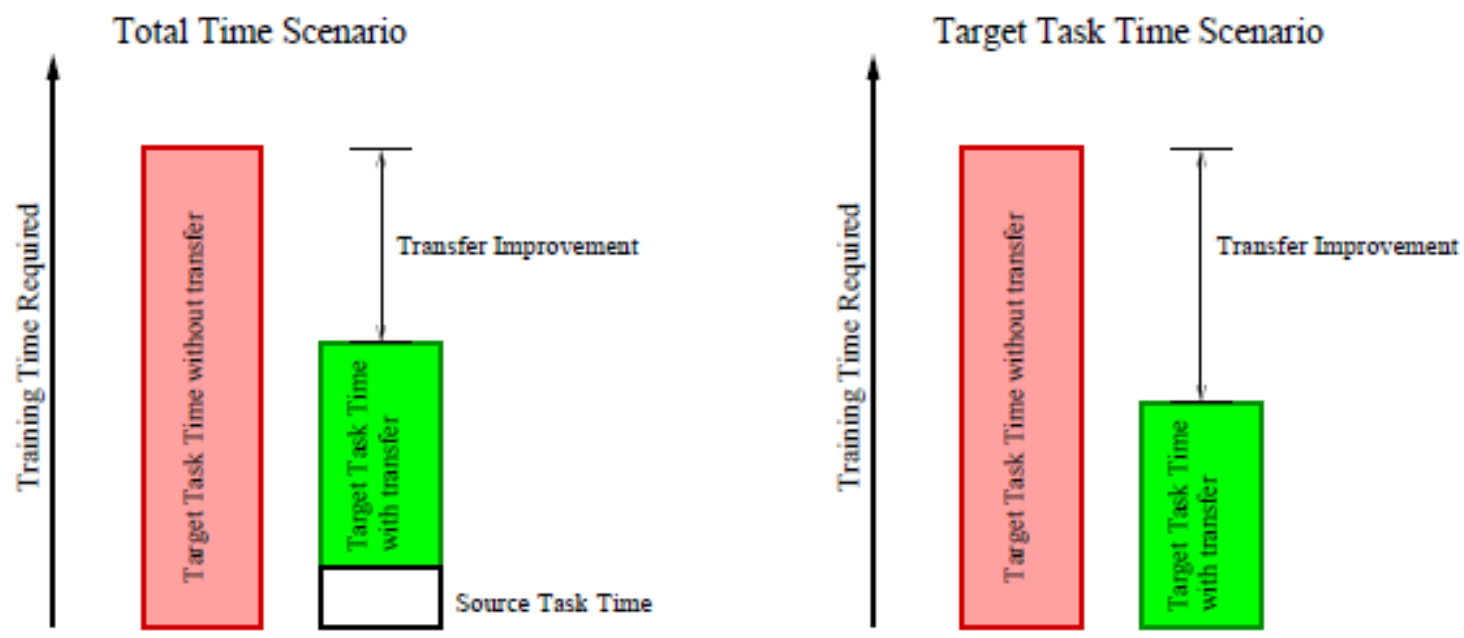

Figure 3.1: Total Time Scenario and Target Task Time scenario in TF learning Source: [7] 
One of the key challenges in transfer learning is to define evaluation metrics. Most evaluation metrics are goal driven. For example, if the goal of transfer is to reduce the overall time required to learn a complex task, then the appropriate evaluation we need to consider is a total learning time scenario. The metric should explicitly includes the time needed to learn the source task [7]. However, if the goal changes to reusing the knowledge from the past to accelerate training in a new task, then only a target task scenario should be considered. In this scenario, we include the time spent on learning in the target task only, not the source task. Thus, the way to treat learning in the source task is not always fixed and changes from scenario to scenario. We show the total time scenario and the target task time scenario in Fig. 3.1. The possible measurement options should always depend on the algorithms focus and the goal of transfer. We conclude the most common metrics as follows [7].

1. Jumpstart: This is the improvement in performance that occurs during the initial phase of a target task learning when transfer learning is applied.

2. Asymptotic Performance: This is the improvement in performance that occurs at the final stage of a target task when transfer learning is applied.

3. Total Reward: The total reward accumulated by an agent in the target task when transfer learning is applied. We compare the accumulated reward with and without transfer learning in the target task

4. Transfer Ratio: This is similar to total reward, but instead of using it directly, we calculate the ratio of the total reward accumulated with and without the transfer in place.

5. Time to Threshold: The elapsed time in the the target task for an agent to achieve a pre-specified performance level when transfer learning is applied. 
Metric 1-4 does not account for the learning time spent by the agent in a source task. However, if a total time scenario is considered, it is natural to use metric 5 since it accounts for time spent learning one or more source tasks. Other metrics may have also been proposed in the literature, but we choose to focus on these five because they are sufficient to describe most transfer learning methods [7]. We present these performance metrics in Fig. 3.2.

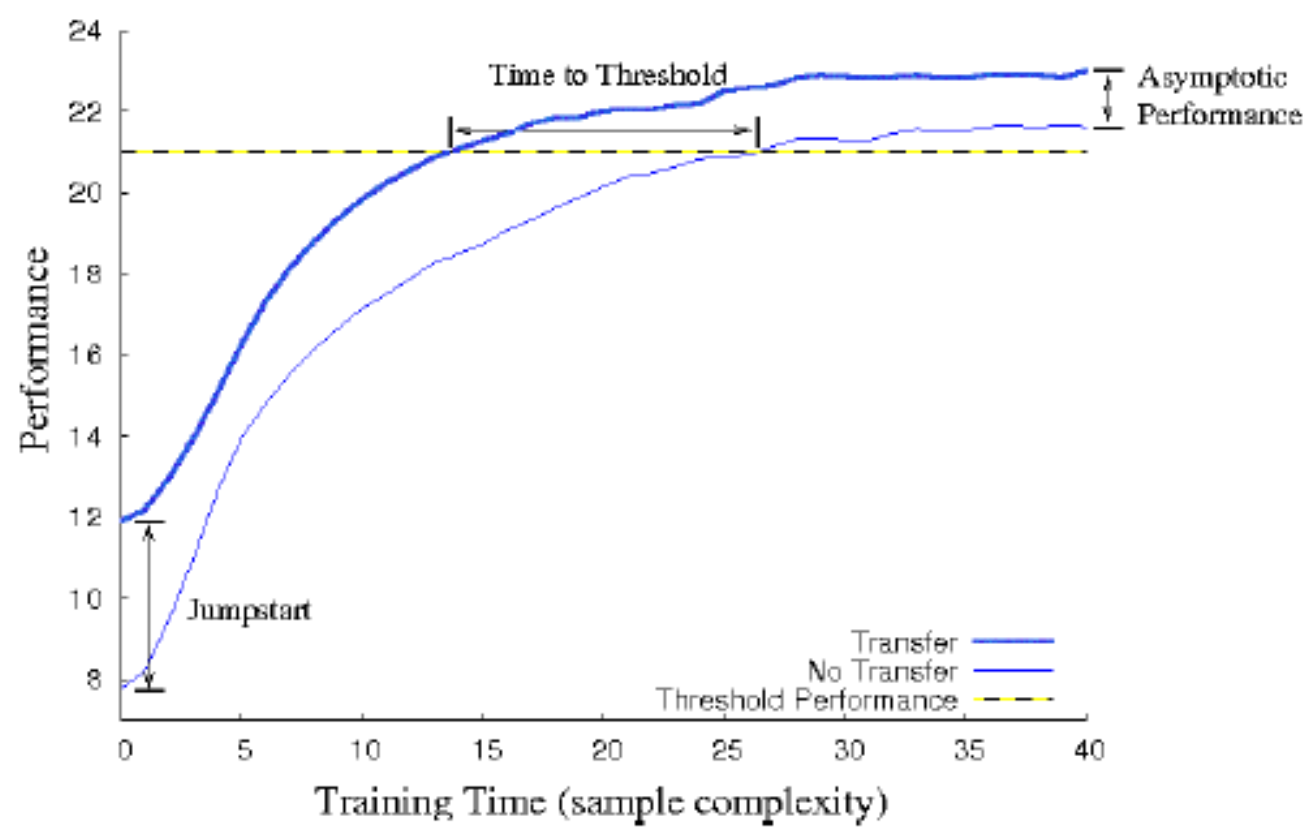

Figure 3.2: Various performance metrics used in TF learning

Source: [7]

\subsubsection{The Five Dimensions of Transfer Learning}

In the literature, transfer learning algorithms for reinforcement learning are mostly categorized based on five dimensions;task difference assumptions, source task selection, task Mappings, transferred Knowledge and allowed learners [7]. We will go through them briefly here. 
Task difference assumptions is the assumption made regarding how the source and target task are different. Various things can be different among the source and target tasks including state-spaces, action-spaces, reward-functions, even the system dynamics and so forth. This assumption has a great impact on which method can be applied in transfer learning among the tasks. For instance, one category of the methods are only suitable when state variables and action set are the same between the source and target task. In one approach a set of tasks are constructed for learners and made incrementally harder not by modifying the dynamics of the tasks, but by moving the agent's initial state further from the goal state [25]. Allowing transfer to occur between less similar source and target tasks gives more flexibility to a human designer in the human-guided scenario. Transfer learning methods that are more flexible have a better chance to apply the past experience in a target task since it allows more difference between source and target tasks. [7].

Source task selection is another major concern that differentiate how transfer learning methods work. Usually human knowledge will be needed to guide the source task selection. However some complex transfer learning algorithms enable agents to select a source task or a set of source tasks autonomously. These kinds of selection mechanism may introduce noise to the learning agent in a target task. This is formally known as negative transfer where transfer hurts the agent's performance. In this scenario, we need make sure the transfer learning algorithms are robust enough to select the right source task or a set of source tasks. The more robust the selection mechanism, the more likely the transfer will provide a benefit [7].

Task Mappings are used when additional detail is needed regarding how the target task and source task are related. We need to investigate the relationship between the target and source task, particularly the state-space and action-space. One of the prominent mappings method is known as inter-task mapping and will be discussed 
in the Section 3.2 and Section 3.3. In most scenarios, we consider the mapping that is provided by human knowledge. However, the mapping needs be learned when the transfer learning is applied autonomously [7].

Transferred Knowledge refers to the type of information being transferred between the source and target task. This kind of information can range from very low-level information (the knowledge of direct control of the agent) to high-level information (information such as how agents function in a particular domain). An example for the low-level information can be the action value of the agent at a particular state, or information contained in weights within function approximators. Whereas an example of high-level information can be how can an agent best learn in a domain. For instance, if we train an agent to balance a pole on a cart, the high-level information in this case can be avoiding hitting the end of the track [8]. Depending on task similarity, different types of knowledge may transfer in a different way. For instance, low-level information is more suitable to transfer among closely related tasks, while high-level concepts are easier to transfer between less similar tasks [7].

Allowed Learners is another concern when the agents in source and target tasks are learning with different algorithms. Some transfer learning methods require agents to use the same reinforcement learning algorithm among source and target tasks. The most flexible method is able to decouple the learning algorithms of agents in the source and target tasks [7].

\subsection{Transfer Learning via Inter-Task Mappings}

Transfer learning via inter-task mappings (TVITM) is a transfer learning technique designed for knowledge transfer between value functions in reinforcement learning agents. This method considers scenarios where the state variables in the source task 
and target task are different [8]. We denote the state in a source task as $S_{\text {source }}$ and $S_{\text {target }}$ is the state in a target task. Thus, $S_{\text {source }} \neq S_{\text {target }}$. Likewise, actions set in

the source and target tasks are also different and we have $A_{\text {source }} \neq A_{\text {target }}$. The goal is to use the learned action value function from the source task $Q_{(\text {source,final })}$ as the initial action-value function $Q_{(\text {target,initial })}$ for the learning agent in a target task. In order to achieve this, a transformation of the action-value function is required such that the learned value can be applied in a target task [8].

We define a transfer functional $\rho$ for such transformation. The environment setup of the source and target task and the transfer functional are illustrated in Fig. 3.3. The transfer functional is used to transform a state-action function $\mathrm{Q}$ from a source task to a target task with different state and action space. Not only a transfer functional $p(Q)$ transfer the action value from a source task, it allows us to apply a policy in a target task [8]. Since a policy generally selects the action which is believed to accumulate the greatest expected total reward, a change in the action value indirectly modify the agent's policy in the target task. Thus, the problem of transforming a policy between two tasks reduces to transforming the action-value function. Defining a transfer functional $\rho$ correctly is the key to enable general TVITM [8].

\subsubsection{TVITM for Keepaway Game}

In the TVITM literature, the transfer learning method is implemented on a few different games. The agents in these games are implemented with different function approximators. In this example, we will briefly go over the Keepaway game and the application of TVITM in this game.

RoboCup simulated soccer is a well explored simulation environment, as it has been the basis of research in reinforcement learning and used widely in this domain. 

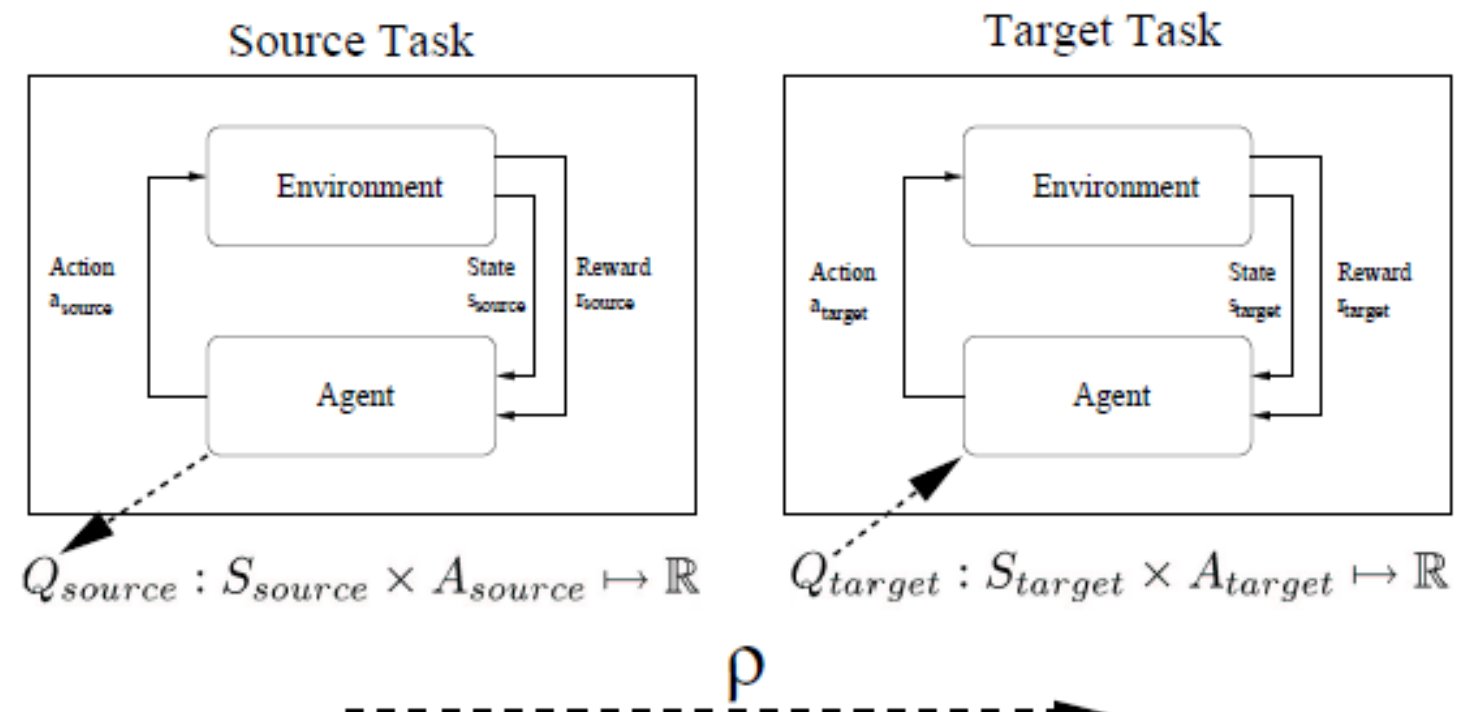

Figure 3.3: The $\rho$ transfer function between tasks

Source: [8]

Although previous work has attempted to use machine learning to learn the full simulated soccer problem [26], it is yet to find a complete solution due to the complexity of the problem. Thus many researchers have divided the RoboCup simulation problem into a few subsets and use machine learning techniques to effectively solve them. The Keepaway game is one of the subproblems. With this approach, we are able to train an agent to learn the action value function of the smaller tasks in Keepaway [8].

In Keepaway, one team, known as keepers, aim to maintain the possession of the ball within a limited region while the other team, known as takers, attempts to steal the ball or force it out of bounds. Whenever the takers take possession or the ball leaves the region, the episode ends. The episode will then reset and the keepers agents will be given possession of the ball again [27]. Reinforcement learning agents in the simulator receive signals regarding the current state of the environment every 150 msec indicating the relative distance and angle to visible objects. These objects can be the ball and other agents. They may execute an action from the action set such 
as the angle to turn, dash with power, or perform a kick at a certain angle every 100 msec [27]. The agents communicate and act in an asynchronous manner while random noise is also introduced in the environment. Full details of the simulator are presented in the RoboCup Soccer Server manual [28]. We show a Keepaway environment with 3 keepers and 2 takers in Fig. 3.4. In the diagram, K1, K2 and K3 are the three keepers

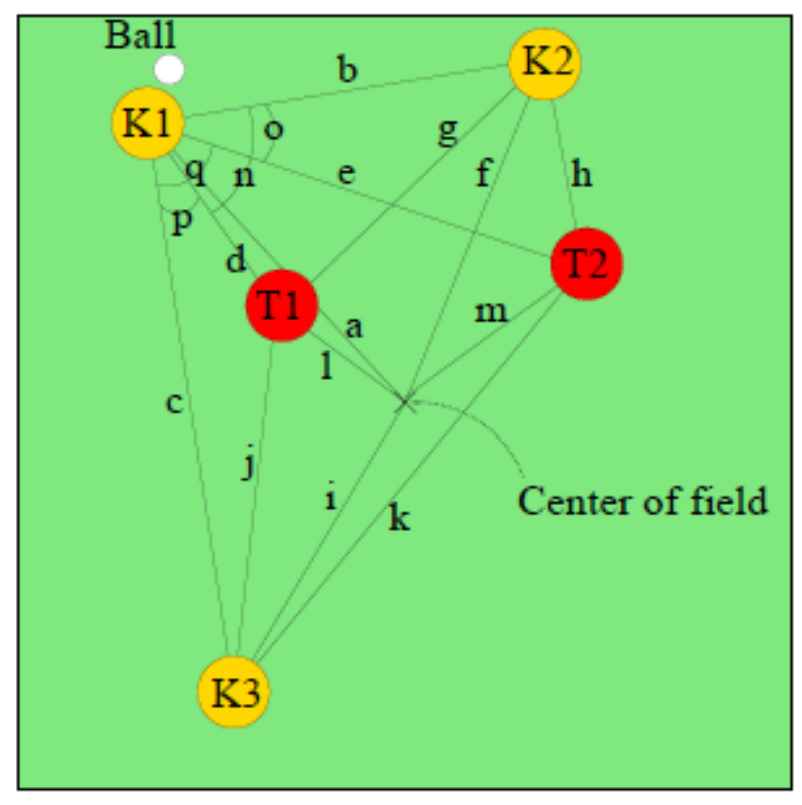
a. $\operatorname{dist}(\mathrm{K} 1, \mathrm{C})$
b. $\operatorname{dist}(\mathrm{K} 1, \mathrm{~K} 2)$
c. $\operatorname{dist}(\mathrm{K} 1, \mathrm{~K} 3)$
d. $\operatorname{dist}(\mathrm{K} 1, \mathrm{~T} 1)$
e. $\operatorname{dist}(\mathrm{K} 1, \mathrm{~T} 2)$
f. dist $(\mathrm{K} 2, \mathrm{C})$
g. $\operatorname{dist}(\mathrm{K} 2 . \mathrm{T} 1)$
h. $\operatorname{dist}(\mathrm{K} 2, \mathrm{~T} 2)$
i. $\operatorname{dist}(\mathrm{K} 3, \mathrm{C})$
j. $\operatorname{dist}(\mathrm{K} 3, \mathrm{~T} 1)$
k. $\operatorname{dist}(\mathrm{K} 3, \mathrm{~T} 2)$
1. $\operatorname{dist}(\mathrm{T} 1, \mathrm{C})$
m. $\operatorname{dist}(\mathrm{T} 2, \mathrm{C})$
n. $\operatorname{ang}(\mathrm{K} 2, \mathrm{~K} 1, \mathrm{~T} 1)$
o. $\operatorname{ang}(\mathrm{K} 2, \mathrm{~K} 1, \mathrm{~T} 2)$
p. ang $(\mathrm{K} 3, \mathrm{~K} 1, \mathrm{~T} 1)$
q. $\operatorname{ang}(\mathrm{K} 3, \mathrm{~K} 1, \mathrm{~T} 2)$

Figure 3.4: The game environment of Keepaway

Source: $[8]$

while $\mathrm{T} 1$ and $\mathrm{T} 2$ are the two takers. A list of state variables used by the agent is also presented on the right hand side of the diagram. The distance between a and $\mathrm{b}$ is denoted as $\operatorname{dist}(a, b)$; the angle made by $\mathrm{a}, \mathrm{b}$, and $\mathrm{c}$, where $\mathrm{b}$ is the vertex, is denoted by ang $(a, b, c)$. These state variables describe the MDP environment perceived by the agent. Fig. 3.5 lists the details about all state variables used for representing the state of 3 vs. 2 Keepaway .

In the TVITM literature, the goal is to transfer knowledge from a learned 3 vs. 2 Keepaway game to a novel 4 vs. 3 Keepaway game [8]. We define the 3 vs. 2 
3 vs. 2 State Variables

\begin{tabular}{|c|c|}
\hline State Variable & Description \\
\hline $\operatorname{dist}\left(K_{1}, C\right)$ & Distance from keeper with ball to center of field \\
\hline $\operatorname{dist}\left(K_{1}, K_{2}\right)$ & Distance from keeper with ball to closest teammate \\
\hline $\operatorname{dist}\left(K_{1}, K_{3}\right)$ & Distance from keeper with ball to second closest teammate \\
\hline $\operatorname{dist}\left(K_{1}, T_{1}\right)$ & Distance from keeper with ball to closest taker \\
\hline $\operatorname{dist}\left(K_{1}, T_{2}\right)$ & Distance from keeper with ball to second closest taker \\
\hline $\operatorname{dist}\left(K_{2}, C\right)$ & Distance from closest teammate to center of field \\
\hline $\operatorname{dist}\left(K_{3}, C\right)$ & Distance from second closest teammate to center of field \\
\hline $\operatorname{dist}\left(T_{1}, C\right)$ & Distance from closest taker to center of field \\
\hline $\operatorname{dist}\left(T_{2}, C\right)$ & Distance from second closest taker to center of field \\
\hline $\operatorname{Min}\left(\operatorname{dist}\left(K_{2}, T_{1}\right)\right.$, dist $\left(K_{2}, T_{2}\right)$ & Distance from nearest teammate to its nearest taker \\
\hline $\operatorname{Min}\left(\operatorname{dist}\left(K_{3}, T_{1}\right), \operatorname{dist}\left(K_{3}, T_{2}\right)\right.$ & Distance from second nearest teammate to its nearest taker \\
\hline $\begin{array}{l}\operatorname{Min}\left(\operatorname{ang}\left(K_{2}, K_{1}, T_{1}\right)\right. \\
\quad \operatorname{ang}\left(K_{2}, K_{1}, T_{2}\right)\end{array}$ & $\begin{array}{l}\text { Angle of passing lane from keeper with ball to } \\
\text { closest teammate }\end{array}$ \\
\hline $\begin{array}{c}\operatorname{Min}\left(\operatorname{ang}\left(K_{3}, K_{1}, T_{1}\right),\right. \\
\operatorname{ang}\left(K_{3}, K_{1}, T_{2}\right)\end{array}$ & $\begin{array}{l}\text { Angle of passing lane from keeper with ball to } \\
\text { second closest teammate }\end{array}$ \\
\hline
\end{tabular}

Figure 3.5: The game environment of Keepaway

Source: $[8]$

Keepaway game as the source task and the 4 vs. 3 Keepaway game as the target task. As discussed earlier, we need to build a transfer functional to map the stateaction function between the pair of tasks. In order to correctly construct the transfer function $\mathrm{p}$, we map the actions and state variables respectively by identifying states or actions that have similar effects on the environment in both tasks [7]. We define $\chi_{A}$ as the inter-task mapping for actions and $\chi_{X}$ as the inter-task mapping for state variables between the source and target task. The formal definition of $\chi_{A}$ and $\chi_{X}$ are presented in the next section.

For the action mapping $\chi_{A}$ in this particular example, "Hold ball" is equivalent in both tasks as it has a similar effect on the environment. Likewise, the action "Pass to closest keeper" is analogous in both tasks as well as "Pass to second closest keeper". The only novel action in the target task "Pass to third closest keeper" is mapped 
to "Pass to second closest keeper" in the source task [8]. Although this mapping requires human intuition and domain expertise, it is reliable as essential information is identified during the transfer.

Likewise, the state variables mapping $\chi_{X}$ is handled with a similar strategy. For example, the novel new state variable "Distance to third closest" in the target task is mapped to "Distance to second closest keeper" in the source task. We present a full description of $\chi_{X}$ in Fig. 3.6. The novel states which are not present in 3 vs. 2 are in bold [8].

Description of $\chi_{X}$ Mapping from 4 vs. 3 to 3 vs. 2

\begin{tabular}{|c|c|}
\hline 4 vs. 3 state variable & 3 vs. 2 state variable \\
\hline dist $\left(K_{1}, C\right)$ & dist $\left(K_{1}, C\right)$ \\
\hline $\operatorname{dist}\left(K_{1}, K_{2}\right)$ & $\operatorname{dist}\left(K_{1}, K_{2}\right)$ \\
\hline $\operatorname{dist}\left(K_{1}, K_{3}\right)$ & $\operatorname{dist}\left(K_{1}, K_{3}\right)$ \\
\hline $\operatorname{dist}\left(\mathbf{K}_{1}, \mathbf{K}_{4}\right)$ & $\operatorname{dist}\left(K_{1}, K_{3}\right)$ \\
\hline $\operatorname{dist}\left(K_{1}, T_{1}\right)$ & $\operatorname{dist}\left(K_{1}, T_{1}\right)$ \\
\hline $\operatorname{dist}\left(K_{1}, T_{2}\right)$ & $\operatorname{dist}\left(K_{1}, T_{2}\right)$ \\
\hline $\operatorname{dist}\left(\mathbf{K}_{1}, \mathbf{T}_{3}\right)$ & $\operatorname{dist}\left(K_{1}, T_{2}\right)$ \\
\hline $\operatorname{dist}\left(K_{2}, C\right)$ & $\operatorname{dist}\left(K_{2}, C\right)$ \\
\hline $\operatorname{dist}\left(K_{3}, C\right)$ & $\operatorname{dist}\left(K_{3}, C\right)$ \\
\hline $\operatorname{dist}\left(\mathrm{K}_{4}, \mathbf{C}\right)$ & $\operatorname{dist}\left(K_{3}, C\right)$ \\
\hline $\operatorname{dist}\left(T_{1}, C\right)$ & $\operatorname{dist}\left(T_{1}, C\right)$ \\
\hline $\operatorname{dist}\left(T_{2}, C\right)$ & $\operatorname{dist}\left(T_{2}, C\right)$ \\
\hline $\operatorname{dist}\left(T_{3}, C\right)$ & $\operatorname{dist}\left(T_{2}, C\right)$ \\
\hline $\operatorname{Min}\left(\operatorname{dist}\left(K_{2}, T_{1}\right), \operatorname{dist}\left(K_{2}, T_{2}\right), \operatorname{dist}\left(\mathbf{K}_{\mathbf{2}}, \mathbf{T}_{3}\right)\right)$ & $\operatorname{Min}\left(\operatorname{dist}\left(K_{2}, T_{1}\right), \operatorname{dist}\left(K_{2}, T_{2}\right)\right)$ \\
\hline $\operatorname{Min}\left(\operatorname{dist}\left(K_{3}, T_{1}\right), \operatorname{dist}\left(K_{3}, T_{2}\right), \operatorname{dist}\left(\mathbf{K}_{3}, \mathbf{T}_{3}\right)\right)$ & $\operatorname{Min}\left(\operatorname{dist}\left(K_{3}, T_{1}\right), \operatorname{dist}\left(K_{3}, T_{2}\right)\right)$ \\
\hline $\operatorname{Min}\left(\operatorname{dist}\left(\mathbf{K}_{4}, \mathbf{T}_{1}\right), \operatorname{dist}\left(\mathbf{K}_{4}, \mathbf{T}_{2}\right), \operatorname{dist}\left(\mathbf{K}_{4}, \mathbf{T}_{3}\right)\right)$ & $\operatorname{Min}\left(\operatorname{dist}\left(K_{3}, T_{1}\right), \operatorname{dist}\left(K_{3}, T_{2}\right)\right)$ \\
\hline $\begin{array}{l}\operatorname{Min}\left(\operatorname{ang}\left(K_{2}, K_{1}, T_{1}\right), \operatorname{ang}\left(K_{2}, K_{1}, T_{2}\right),\right. \\
\left.\operatorname{ang}\left(\mathbf{K}_{\mathbf{2}}, \mathbf{K}_{\mathbf{l}}, \mathbf{T}_{3}\right)\right)\end{array}$ & $\operatorname{Min}\left(\operatorname{ang}\left(K_{2}, K_{1}, T_{1}\right), \operatorname{ang}\left(K_{2}, K_{1}, T_{2}\right)\right)$ \\
\hline $\begin{array}{l}\operatorname{Min}\left(\operatorname{ang}\left(K_{3}, K_{1}, T_{1}\right), \operatorname{ang}\left(K_{3}, K_{1}, T_{2}\right),\right. \\
\left.\operatorname{ang}\left(\mathbf{K}_{3}, \mathbf{K}_{\mathbf{l}}, \mathbf{T}_{3}\right)\right)\end{array}$ & $\operatorname{Min}\left(\operatorname{ang}\left(K_{3}, K_{1}, T_{1}\right), \operatorname{ang}\left(K_{3}, K_{1}, T_{2}\right)\right)$ \\
\hline $\begin{array}{l}\operatorname{Min}\left(\operatorname{ang}\left(\mathbf{K}_{4}, \mathbf{K}_{1}, \mathbf{T}_{1}\right), \operatorname{ang}\left(\mathbf{K}_{4}, \mathbf{K}_{1}, \mathbf{T}_{\mathbf{2}}\right),\right. \\
\left.\operatorname{ang}\left(\mathbf{K}_{4}, \mathbf{K}_{1}, \mathbf{T}_{3}\right)\right)\end{array}$ & $\operatorname{Min}\left(\operatorname{ang}\left(K_{3}, K_{1}, T_{1}\right), \operatorname{ang}\left(K_{3}, K_{1}, T_{2}\right)\right)$ \\
\hline
\end{tabular}

Figure 3.6: Mapping between states in 4 vs. 3 to states in 3 vs. 2 . Source: $[8]$ 


\subsubsection{Transfer Functional Structure}

In this subsection we formally define the inter-task mapping between a source and a target task and how a transfer functional $\rho$ is constructed. One prerequisite for the transfer functional to work is that the source and target task must be related to some degree. Otherwise, the transfer will not lead to any improvement [8]. Not only do the two tasks have to be related, but also understanding how are they related is important in order to make the transfer successful. A pair of inter-task mappings, denoted $\chi_{X}$ and $\chi_{A}$, are used to represent the relation between the pair of tasks [8]. We map the state variables in the target task to the most similar state variables in the source task :

$$
\chi_{X}\left(x_{i, \text { target }}\right)=x_{j, \text { source }}
$$

Similarly, we perform mapping on the actions between the pair of tasks:

$$
\chi_{A}\left(a_{i, \text { target }}\right)=a_{j, \text { source }}
$$

These two mapping, $\chi_{X}$ and $\chi_{A}$, are used to construct the transfer functional $\rho$. We illustrate the construction with the two mapping in Fig. 3.7, where $\chi_{X}$ and $\chi_{A}$ are mappings from a target task to a source task and $\rho$ maps an action-value function from a source task to a target task. There can be multiple transfer functionals $\mathrm{p}$ defined for the same pair of tasks depending on what learning algorithms are used by the agents. For instance, agents may use different function approximators in

the two tasks. We consider the type of the information involved in the process of transferring the state-action value function as low-level information among the two tasks. This type of information is encoded with the most task-specific part of the 
learner's knowledge, such as weights in neural network function approximators [8].

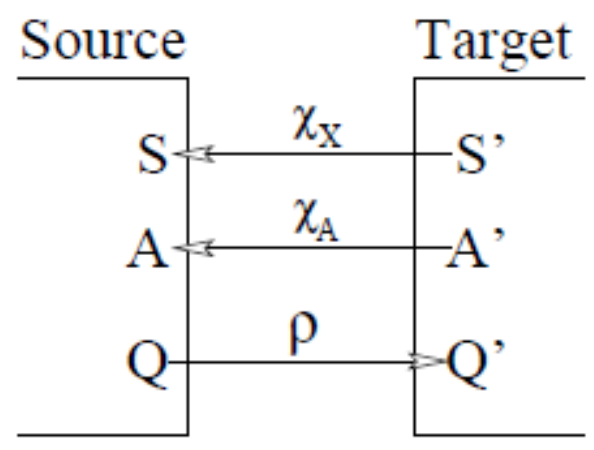

Figure 3.7: The use of mapping $\chi_{x}$ and $\chi_{a}$ in $\rho$

Source: [8]

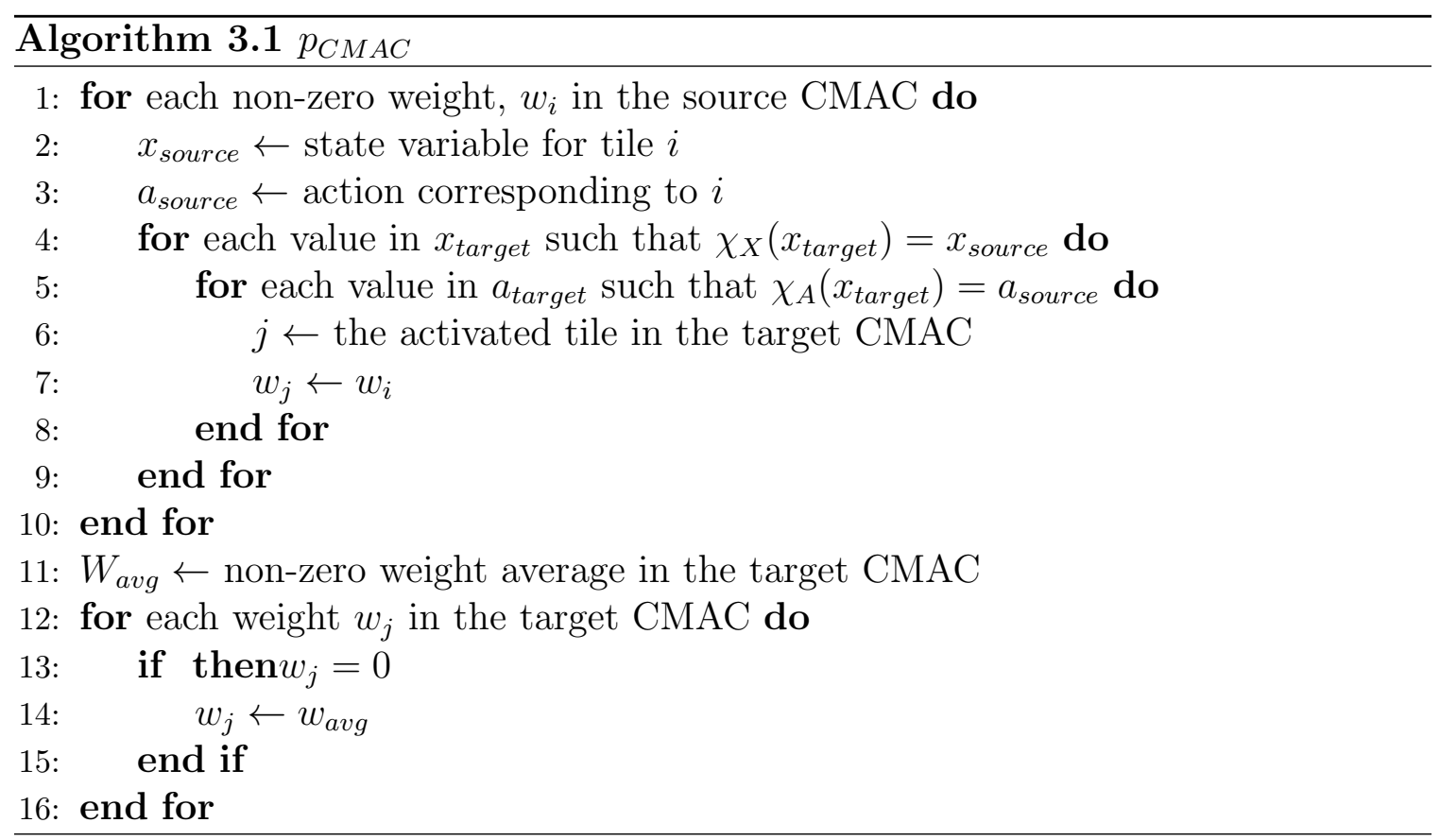

\subsubsection{TVITM for Different Function Approximators}

Many function approximators can be used by agents to learn the game Keepaway [8]. One approach is the linear tile-coding function approximation, also known as 
a CMAC (cerebellar model arithmetic computer). This function approximator has been successfully used in many reinforcement learning systems [29], including past Keepaway research [30]. A $p_{C M A C}$ is constructed to use the learned action value in the final state of the 3 vs. 2 game to initialize the weights for the activated tiles in 4 vs. 3 task. This can be achieved by copying the weights learned in the source CMAC into weights in a newly initialized target CMAC, using $\chi_{X}$ and $\chi_{A}$. Another implementation uses radial basis function approximation (RBF) [7]. The $p_{R B F}$ is constructed similarly to the $p_{C M A C}$ with a similar weight structure. The major difference between the RBF and CMAC function approximators is the weights summing process. Both function approximators have the similar structure in weights [8]. The details of this procedure is described in Algorithm 3.1 and more details can be found in literature [8].

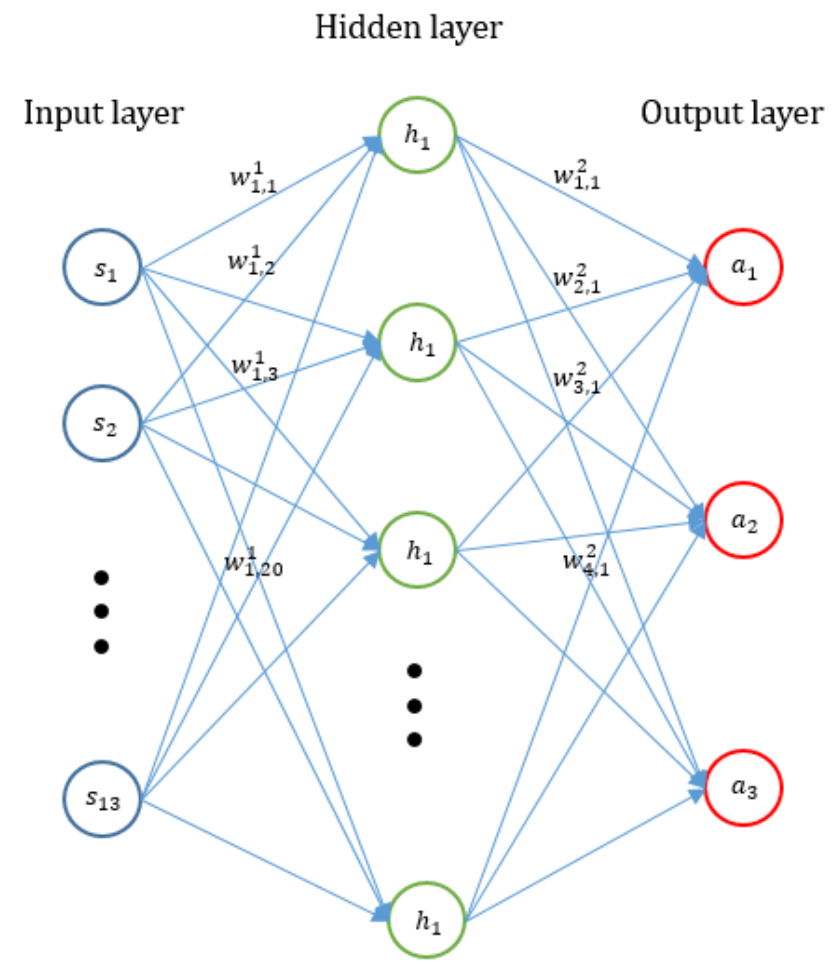

Figure 3.8: 13-20-3 Neural Network structure in the source task 


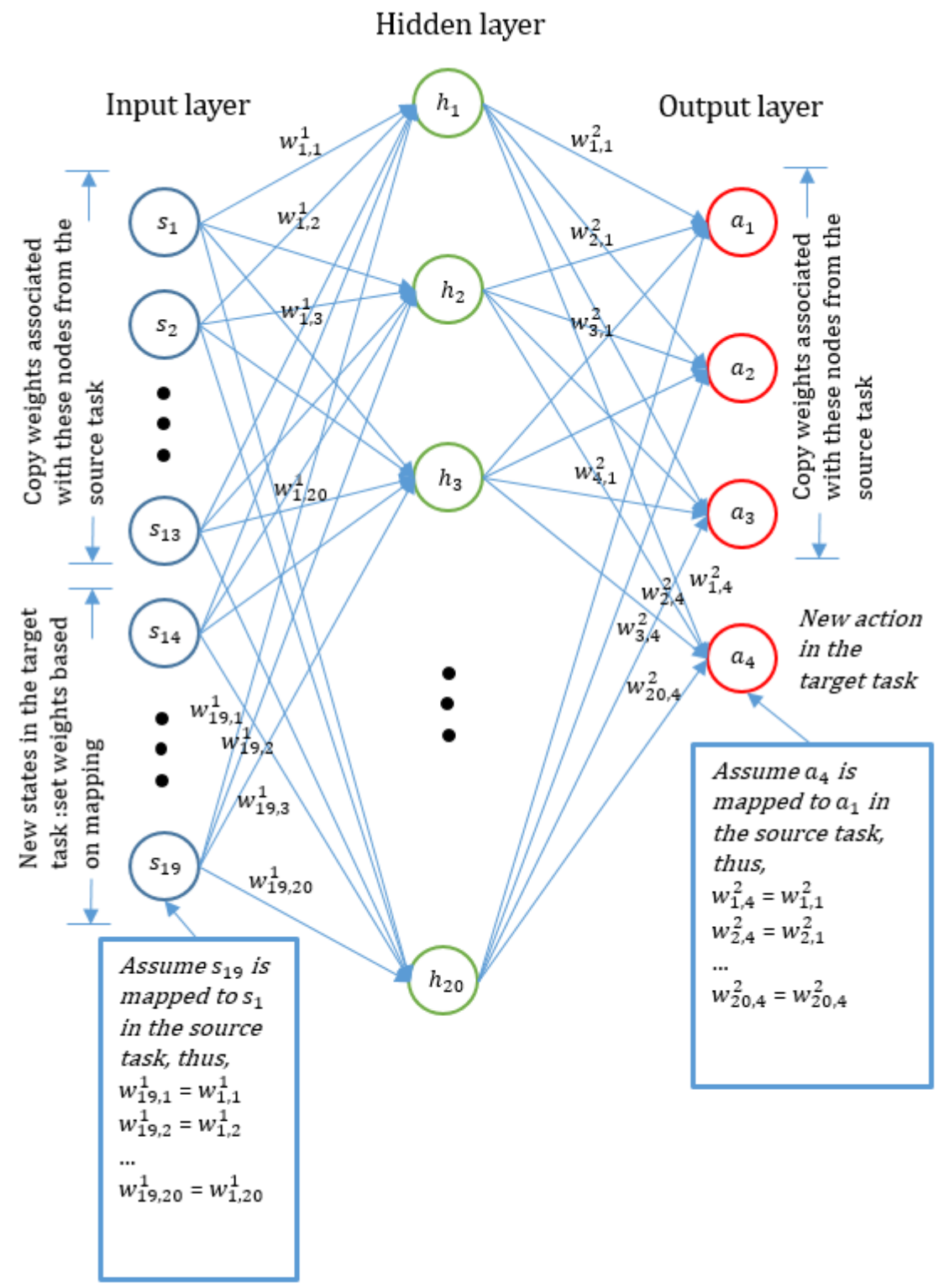

Figure 3.9: 19-20-4 Neural Network structure in the target task

The third approach is to use an artificial neural networks (ANN) as the function approximator. First we train the agent using a 13-20-3 ANN in the source task, where 13 is the number of input nodes, 20 is the number of hidden nodes and 3 is the number of output nodes. To enable transfer, we then augment the ANN to a 19-20-4 structure in the target task by adding 6 inputs and 1 output node [8]. The additional input and 
output nodes represent new states and actions in the target task respectively. The weights between inputs 1-13 and the hidden nodes are directly copied over from the 13-20-3 network. Likewise, the weights from hidden nodes to outputs 1-3 are directly copied over to the 19-20-4 network [8]. The rest of the weights are copied according to their location and based on $\chi_{X}$ and $\chi_{A}$. We illustrate the neural network structure and the weight mapping in the source and target task respectively in Fig. 3.8 and Fig. 3.9, where $s_{14}-s_{19}$ are the input nodes for the 6 new state variables and $a_{4}$ is the output node for the new action. The new states and action are mapped to the most similar states and actions in the source task as defined in 3.1 and 3.2. We formally define the function $\psi$ to map nodes in the two ANN between the pair of tasks:

$$
\psi(n)= \begin{cases}\chi_{X}(n), & \text { if } n \text { is an input } \\ \chi_{A}(n), & \text { if } n \text { is an output } \\ \delta(n), & \text { if } n \text { is a hidden node }\end{cases}
$$

where a function $\delta$ represents $\delta\left(h_{\text {target }}\right)=h_{\text {source. }}$ (e.g. $\delta$ ("nth hidden node in the target network") = "nth hidden node in the source network."). We summarize this process in detail in Algorithm 3.2 [8].

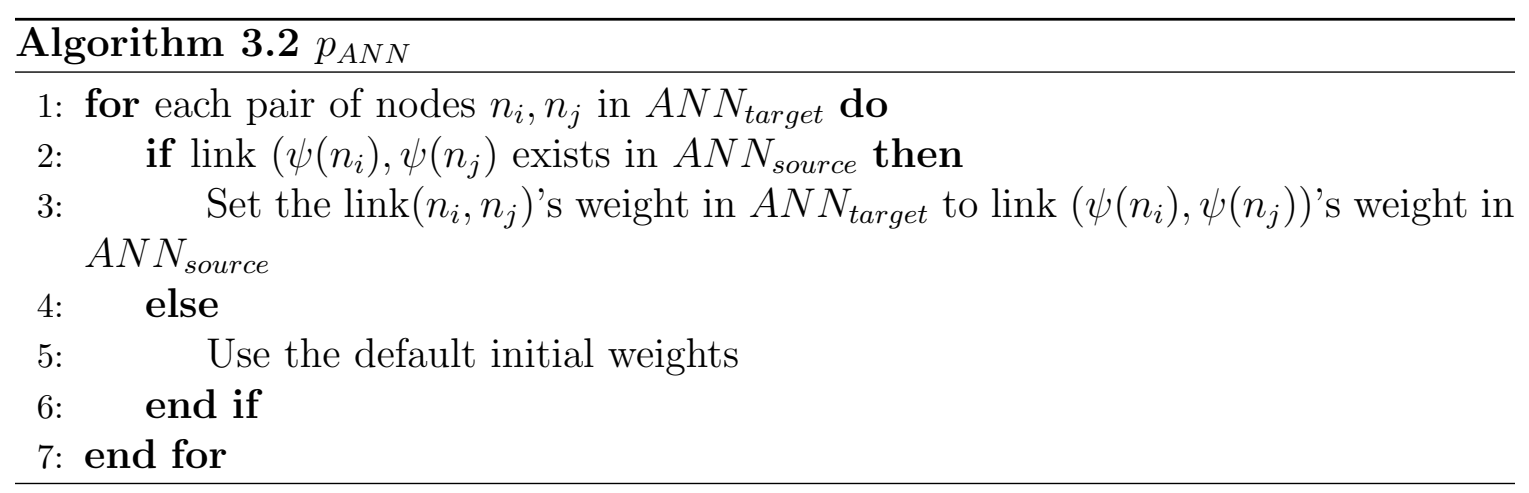




\subsection{Transfer via InterTask Mappings in Policy Search}

While the previous TVITM methods have focused on transferring the state-action value functions between pairs of tasks, many other approaches target at transferring policies across tasks with different state and action space. One of the notable variants is known as transfer via inter-task mappings for policy search methods (TVITM-PS), in which a transfer functional is constructed to transfer the learned policies from a trained neural network function approximator in the source task to a novel neural network in the target task [9].

The process of constructing the policy transfer functional $p_{\text {policy }}$ is very similar to those in the state-action value transfer. The functional is constructed based on the relationship between state variables and actions in the pair of source and target tasks such that $p_{\text {policy }}\left(\pi_{\text {source }}\right)=\pi_{\text {target }}$. One prerequisite in this process is the sufficient domain knowledge or experience. We assume two inter-task mappings are provided by a human: $\chi_{\text {policy }, X}$ and $\chi_{\text {policy }, A} \cdot \chi_{\text {policy,X }}$ maps each state variable in the target task to the most similar state variable in the source task [9]:

$$
\chi_{\text {policy }, X}\left(x_{i, \text { target }}\right)=x_{j, \text { source }}
$$

Similarly, we perform mapping on the actions between the pair of tasks using $\chi_{\text {policy }, A}$ :

$$
\chi_{\text {policy }, A}\left(a_{i, \text { target }}\right)=a_{j, \text { source }}
$$

In a ANN action selector, the input nodes represent state variables while output nodes represent actions. This is different from the action value function approximator ANN where each output represents an action value. The neural network construction and 
the weight mapping between the source and target tasks is similar to the example shown in Fig. 3.8 and Fig. 3.9 in section 3.2.3. Formally, the mapping between a node in the target ANN to a node in the source ANN via the function $\psi$ is defined as [9],

$$
\psi(n)= \begin{cases}\chi_{\text {policy }, X}(n), & \text { if } n \text { is an input } \\ \chi_{\text {policy, } A}(n), & \text { if } n \text { is an output } \\ \delta(n), & \text { if } n \text { is a hidden node }\end{cases}
$$

where a function $\delta$ represents $\delta\left(h_{\text {target }}\right)=h_{\text {source. }}$ (e.g. $\delta$ ("nth hidden node in the target network") = "nth hidden node in the source network."). We present this process in full detail in Algorithm 3.3 [9].

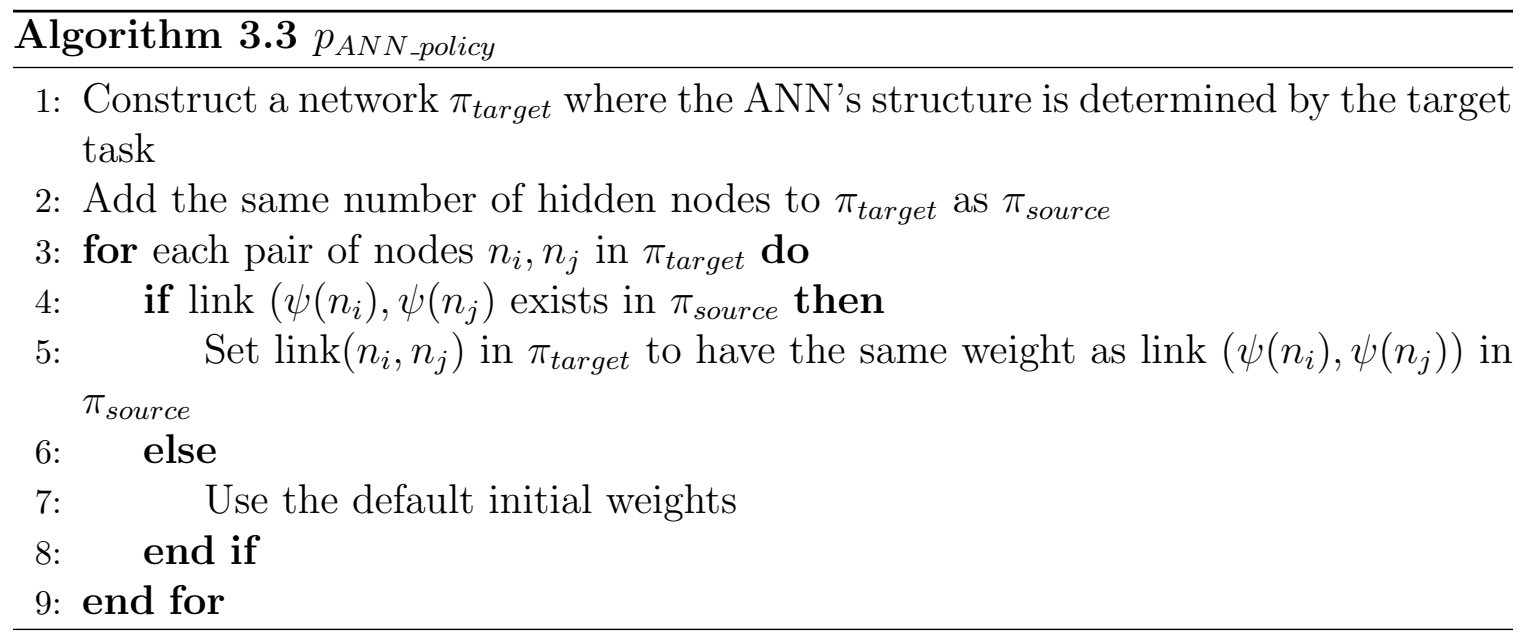

\subsection{Transfer Learning Example in the Paddle Game}

For better illustrating the concept, we have applied transfer learning to one of the well-known Atari games in this section. The Atari game we use is a simple paddle and ball game. There are two elements in the game, a moving ball and a paddle. 
The paddle is on the ground and can only move left or right. The goal of the game is to move the paddle such that the moving ball is hit before touching the ground. If the ball touches the ground instead of the paddle, that's a miss and the game is over. If the ball touches the paddle it bounces back and the game continues. The more hits the paddle scores, the better the game is played. We present a snapshot of the game in Fig. 3.10 and we use this game as our source task. For the target task, the environment is changed in way that a second ball is introduced while the game mechanics remains the same: move the paddle to hit the balls as much as possible. Fig. 3.11 shows a snapshot of the target task.

The environment is set up with built-in turtle module in python. The turtle module provides many useful functions and commands to draw intricate shapes and pictures [31]. In order to be consistent with the literature, the agent in the source task is trained with an artificial neural network (ANN) as a function approximator for

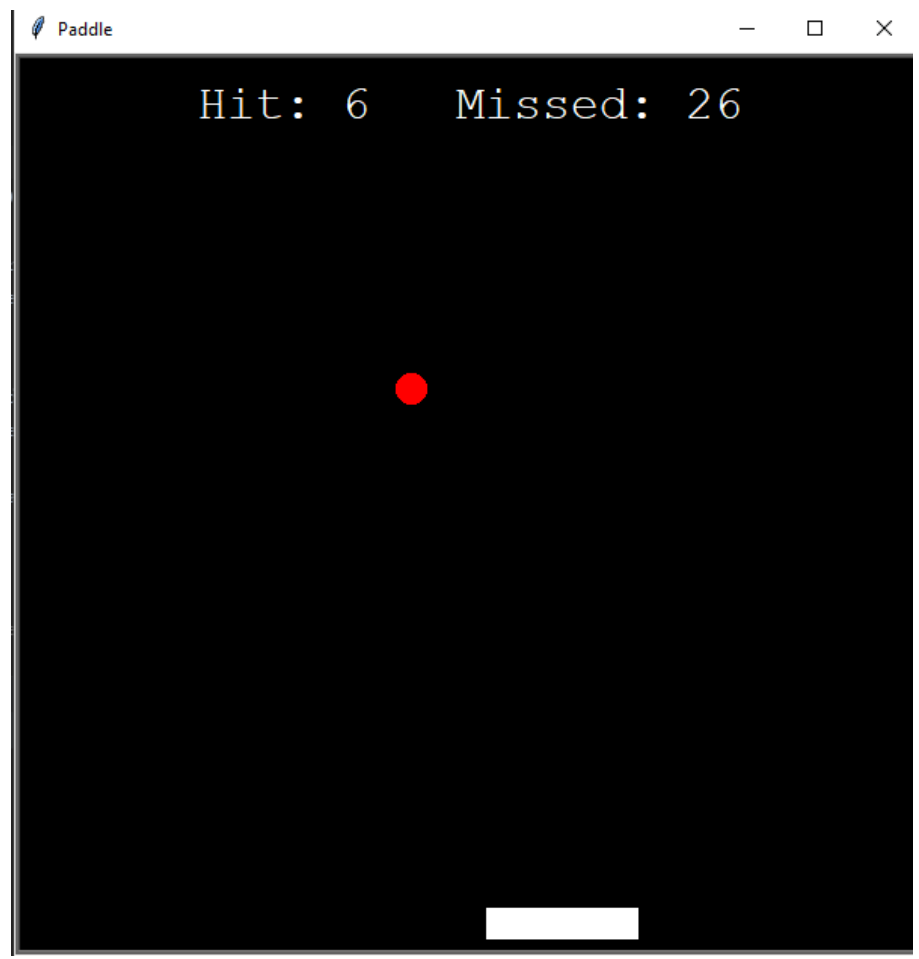

Figure 3.10: Paddle game with 1 ball 


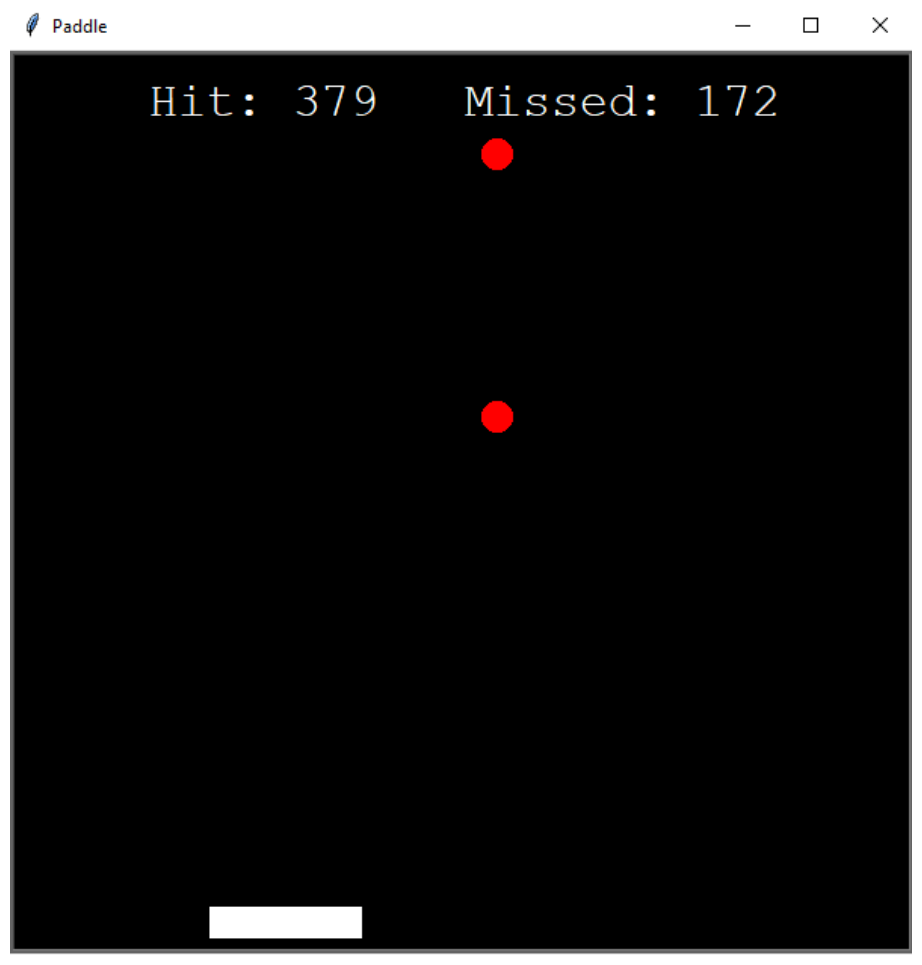

Figure 3.11: Paddle game with 2 balls

the action value. In latest research, we sometimes refer to a neural network function approximator estimating the action value as a Q-network [12]. The action space for the agent is simple, move left, move right, or do nothing: $A=[$ left, right,wait $]$. We design the rewards such that

- the agent receives a reward of +5 if the ball is hit by the paddle

- the agent receives a reward of -5 if the game is lost

- the agent receives a reward of -0.1 if the paddle moves. This is for discouraging unnecessary movement

The state variables to the ANN function approximator has the following design:

- State $s_{1}$ : position of the paddle in the $\mathrm{x}$ axis

- State $s_{2}$ : position of the ball in the $\mathrm{x}$ axis 
- State $s_{3}$ : position of the ball in the y axis

- State $s_{4}$ : velocity of the ball in the $\mathrm{x}$ axis

- State $s_{5}$ : velocity of the ball in the y axis

Each episode has a maximum of 2000 time steps. At each time step, the agent will select an action and receive the next states information and reward from the environment. An episode ends if the game is lost when the ball touches the ground or the maximum time step is reached.

The ANN function approximator in the source task has the structure of 5-20-3. This represents a neural network with 5 inputs and 3 outputs with a hidden layer of 20 neurons. In the target task, the 5-20-3 ANN is augmented by adding 4 different inputs while the number of hidden nodes and output remain unchanged. The addition of the 4 different inputs is introduced by new state variables. We show the design of new state variables in the target task as follow:

- State $s_{1}$ : position of the paddle in the $\mathrm{x}$ axis

- State $s_{2}$ : position of the ball1 in the $\mathrm{x}$ axis

- State $s_{3}$ : position of the ball1 in the $\mathrm{y}$ axis

- State $s_{4}$ : velocity of the ball1 in the $\mathrm{x}$ axis

- State $s_{5}$ : velocity of the ball1 in the y axis

- State $s_{6}$ : velocity of the ball2 in the $\mathrm{x}$ axis

- State $s_{7}$ : velocity of the ball2 in the y axis

- State $s_{8}$ : velocity of the ball2 in the $\mathrm{x}$ axis 
- State $s_{9}$ : velocity of the ball2 in the y axis

As described in the five dimensions of transfer learning in section 3.1.2, the source and target tasks differ in the state variables, whereas the action space are identical between the pair of tasks in this case. The source task selection and task mappings require human knowledge and we choose the inter-task mapping as our mapping method. Since the agents in the source and target tasks are both learning with ANN function approximation, we aim to transfer the low-level information, which is the weights of the nodes in ANN, between the pair of tasks.

For the inter-task mapping, we map the states in the target task to the most relevant states in the source task. The similar mapping process is described with graphic details in section 3.2.3. The states $s_{1}-s_{5}$ in the target task are mapped to states $s_{1}-s_{5}$ in the source tasks while states $s_{6}-s_{9}$ in the target tasks are mapped

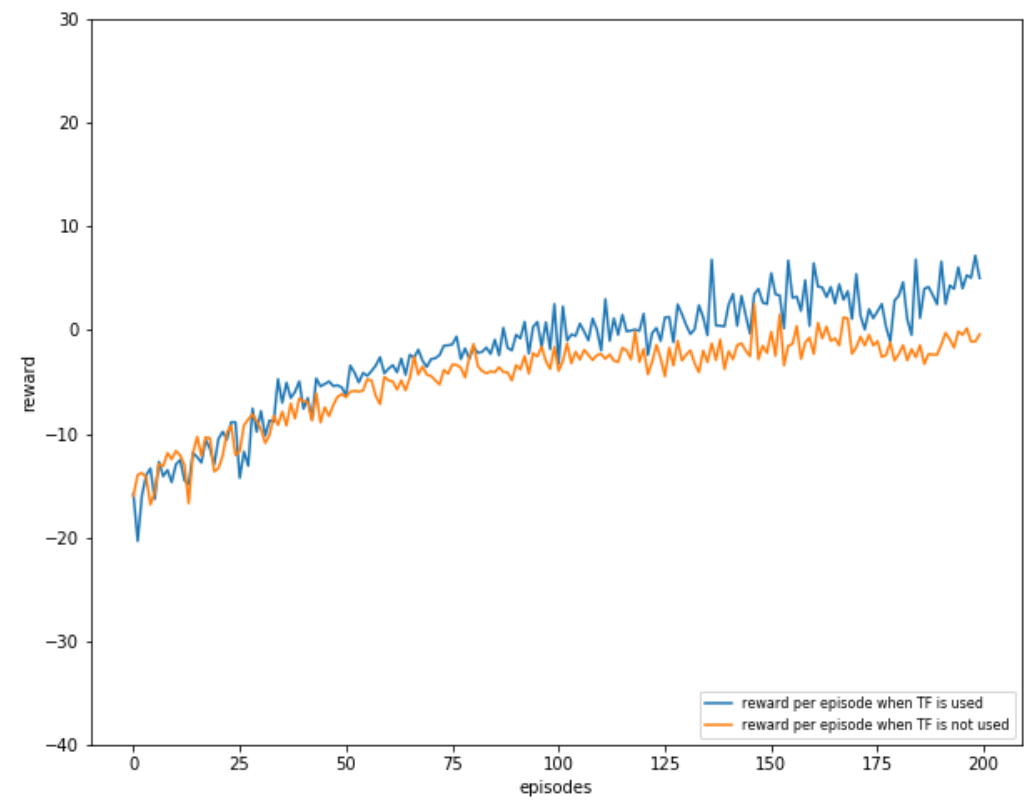

Figure 3.12: Reward per episode between TF and non-TF case 


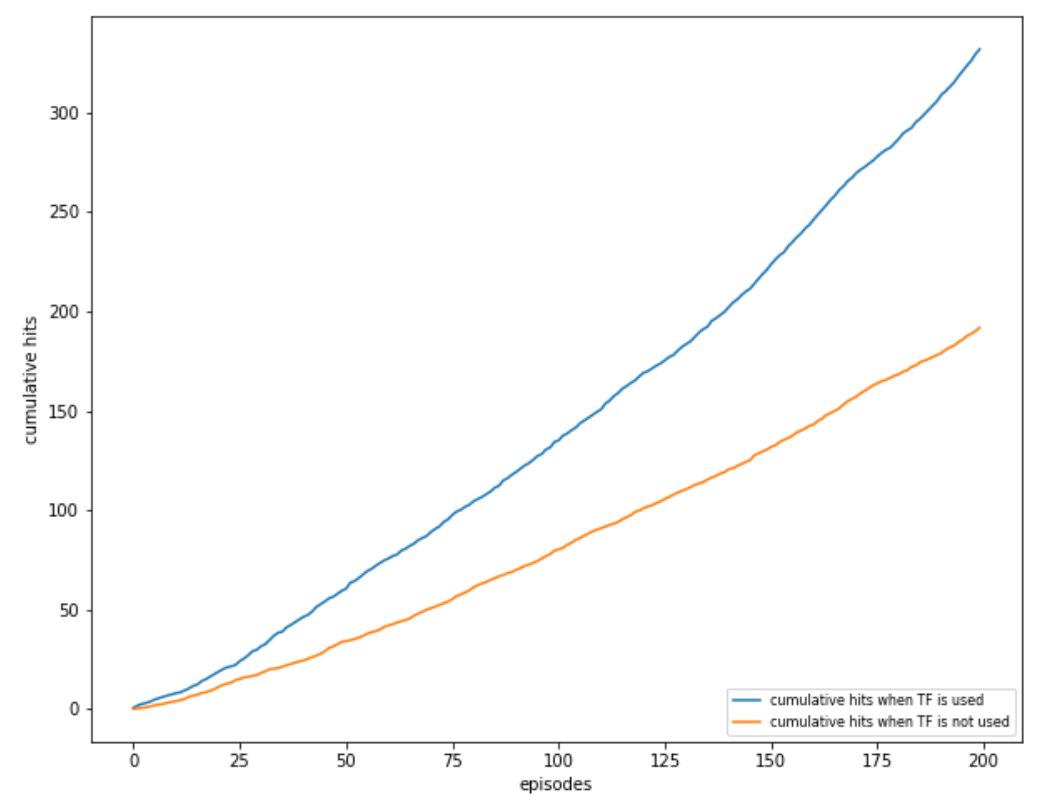

Figure 3.13: Cumulative hits between $\mathrm{TF}$ and non-TF case

to the $s_{2}-s_{5}$ in the source tasks respectively. To implement the mapping in ANN function approximator, we follow the process described in Algorithm 3.2. The weight between input 6 - 9 and the hidden nodes are directly copied over from the 5-20-3 network in the source task. The rest of the weights are copied according to their location and based on the mapping functional. We train the agent with and without using the transfer learning in 10 game trials, 200 episodes each respectively.

Fig. 3.12 presents the total reward the agent collects in each episode, averaged over 10 games (trials) for both TF and non-TF scenarios and Fig. 3.13 highlight the cumulative ball hits by the agent, averaged over 10 games (trials) for both TF and non-TF scenarios. We observe that the agent is able to collect more rewards and performs more hits when transfer learning is used. Therefore, a clear improvement in performance is observed. Furthermore, we are also interested in knowing how the 


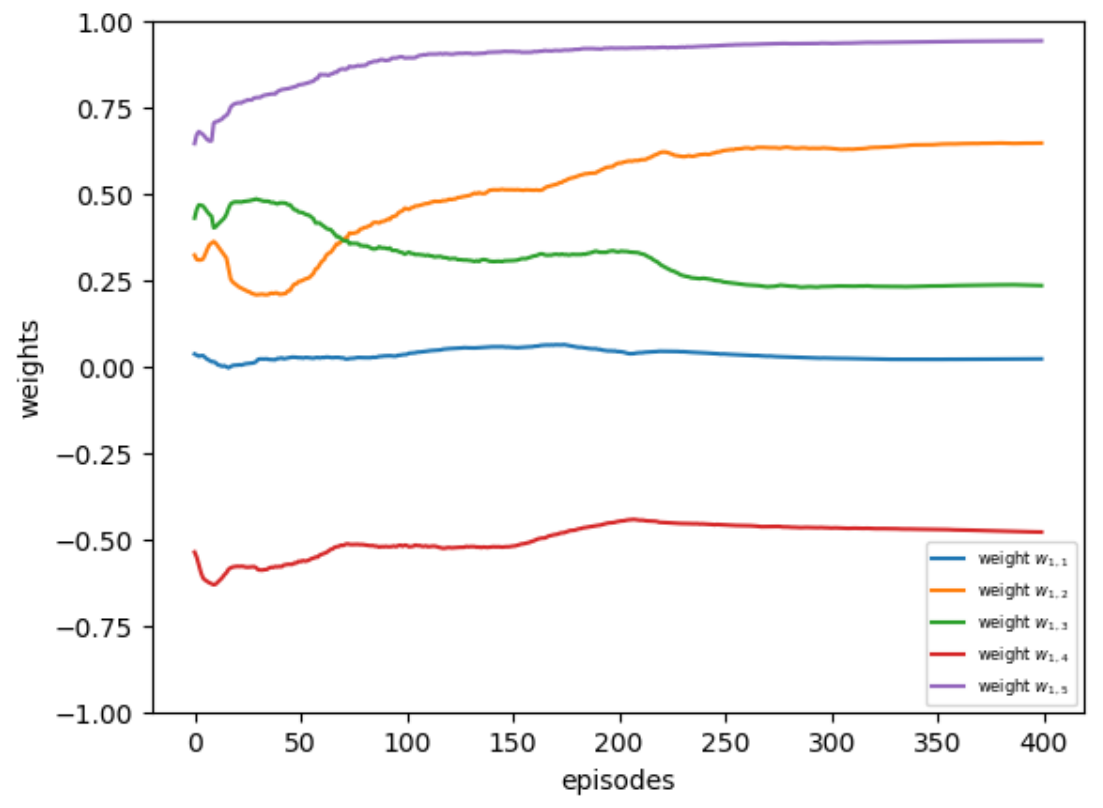

Figure 3.14: NN weights with TF

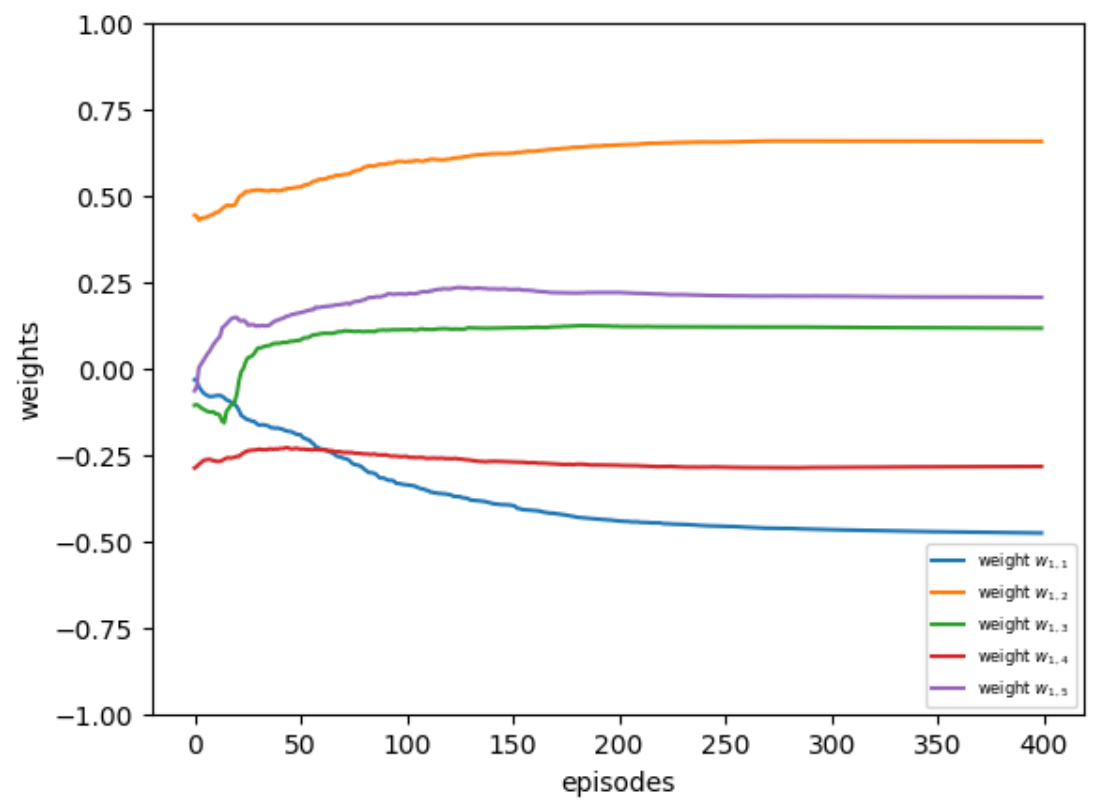

Figure 3.15: NN weights without TF 
weights converge during the training. We pick 5 arbitrary weights from the first NN layer and present their trajectories in Fig. 3.14 and Fig. 3.15 respectively for the transfer and non-transfer scenario. As can be seen all weights converge to different values after 400 episodes since they are initialized randomly in the beginning.

\subsection{Summary}

In this chapter, we provided a brief introduction to transfer learning. We first presented an overview of the transfer learning. In the first section we reviewed the evaluation for transfer learning. We then discussed the five dimensions of transfer learning.

In the second section, we briefly reviewed one of the notable method, transfer via inter-task mappings. The fundamental transfer functional structure was introduced. We also presented a detailed example that applies the TVITM method. While in the next section, a similar approach, transfer via inter-task mappings in policy search, was introduced to perform the knowledge transfer in the policy search and action selection.

In the last section, we included one detailed example of applying the aforementioned transfer learning method to the reinforcement learning agent with ANN function approximator. The concepts presented in this chapter will be used in later chapter to design our novel transfer learning method in the fuzzy actor-critic learning domain. 


\section{Chapter 4}

\section{Transfer Learning in the FACL Algorithm}

Temporal difference (TD) based learning methods have shown some success in many reinforcement learning tasks because of their ability to learn in an environment that has limited prior knowledge and minimal feedback. However, the basic TD algorithms have been exhibiting slow convergence speed to near-optimal behaviors for learning agents [8]. The fuzzy actor-critic learning algorithm presented in chapter 1 is also a TD based learning method since the actor and critic are both adapted based on temporal difference. In chapter 3 we have introduced transfer learning in RL in which we can speed up the agent's learning process by applying previous experience in a new task. In this chapter we investigate the specific scenario, namely, the transfer learning in fuzzy actor-critic learning algorithm [32]. Inspired from transfer via intertask mapping (TVITM) and transfer via inter-task mapping for policy search methods (TVITM-PS), we propose a novel fuzzy rule transfer (FRT) method to map fuzzy rules between the source task and target task within the unique FIS structure in FACL. We simulate and evaluate our method in a few differential games, such as the pursuit - evasion game and the guarding-a-territory game, covering both single agent and multi-agent scenarios. These differential games represent simulation of real-word scenario and have been studied in our previous research [16], [18], [33]. 


\subsection{Fuzzy Rule Transfer in FACL}

We propose a novel method to transfer knowledge between the learned and target task, particularly in the fuzzy actor-critic learning algorithm, via the fuzzy rule transfer(FRT) method. For the FACL algorithm used in this work, both critic and actor are implemented with zero-order Takagi-Sugeno-Kang (TSK) FISs with constant consequent parameters. Each FIS consists of L rules. The inputs of each rule are $n$ fuzzy input variables, whereas the consequent of each rule is a constant number. Each rule $l(l=1, \ldots, L)$ has the following form (4.1).

$$
R_{l}: \quad I F \quad s_{1} \text { is } A_{1}^{l}, \ldots, \text { and } s_{n} \text { is } A_{n}^{l} \text { Then } z_{l}=k_{l}
$$

where the variable $z_{l}$ represents the output variable of rule $l$, and $k_{l}$ is the consequent parameter of rule $l$. The variable $s_{i}(i=1, \ldots, n)$ is the $i$ th input state variable of the fuzzy system, and $\mathrm{n}$ is the number of input state variables. The $A_{i}^{l}$ represents the linguistic value of the input $s_{i}$ at the rule $l$.

The total number of rules can be derived based on the number of input variables and membership functions (MF). We denote $h_{i}$ as the number of membership functions for each state variable $s_{i}$. Given $n$ input state variables, the total number of rules $L$ can be calculated as Eq.(4.2).

$$
L=h_{1} \times h_{2} \times h_{3} \ldots \times h_{n}
$$

In this work we consider the typical case where the input states are different in the 
source and target tasks. This means the rules are also different between the tasks. As described earlier, we use $L_{\text {source }}$ as the total number of rules in the source task and $L_{\text {target }}$ as the total number of rules in the target task. Thus the consequent parameter for source and target tasks are $k_{i}\left(i=1, \ldots, L_{\text {source }}\right)$ and $k_{j}\left(j=1, \ldots, L_{\text {target }}\right)$ respectively. We consider $L_{\text {target }}$ to be much larger than $L_{\text {source }}$ as target tasks are assumed to have more complex states than the source tasks.

Our goal is to enable knowledge transfer between the source and target tasks. In FACL, the learned knowledge is embedded in the consequent parameters in the FIS. Due to the difference in rule numbers explained earlier, we derive a mapping functional $\psi$ to map the initial consequent parameters in a target task to the learned consequent parameter in a source task based on the similarity of the rules. Initializing the FIS in a target task with the learned knowledge allows an immediate improvement in the state estimation of the critic and action selection of the actor during the initial learning phase. This improvement leads the learner to take the actions that are more reasonable over the randomly selected actions, which boosts the learning speed.

Similar to the mapping methods in TVITM and TVITM-PS described in Chapter 3, we perform mapping $\psi$ on both the critic and the actor of the agent in FACL. The difference is that the mappings are performed on the state variables and actions in TVITM and TVITM-PS, whereas in FACL the mapping is directly performed on the fuzzy rules in the critics and actors between the source and target task. The consequent parameters of fuzzy rules in a target task's critic FIS is mapped to the learned consequent parameters of the most similar rules in a source task's critic FIS, via $\psi_{\text {critic }}$ in (4.3)

$$
\psi_{\text {critic }}\left(k_{i, \text { target }}^{\text {critic }}\right)=k_{j, \text { source }}^{\text {critic }}
$$


Where $k_{i, \text { target }}^{\text {critic }}\left(i=1, \ldots, L_{\text {target }}\right)$ is the consequent parameter of the critic's FIS in a target task and $k_{j, s o u r c e}^{\text {critic }}\left(j=1, \ldots, L_{\text {source }}\right)$ is the consequent parameter of the critic's FIS in a source task. Furthermore, we use $\psi_{\text {actor }}$ to map the consequent parameters of fuzzy rules in the actor's FIS in a target task to the learned consequent parameters of the most similar rules in the actor's FIS in a source task, as given in (4.4)

$$
\psi_{\text {actor }}\left(k_{i, \text { target }}^{\text {actor }}\right)=k_{j, \text { source }}^{\text {actor }}
$$

Where $k_{i, \text { target }}^{\text {actor }}\left(i=1, \ldots, L_{\text {source }}\right)$ is the consequent parameter of the actor's FIS in a target task and $k_{j, \text { source }}^{\text {actor }}\left(j=1, \ldots, L_{\text {target }}\right)$ is the consequent parameter of the actor's FIS in a source task. As can be seen, both mappings are performed based on the similarity of the fuzzy rules between the source and target tasks. We will discuss the fuzzy rules similarity in the next section.

\subsubsection{Similarity between Fuzzy Rules}

One prerequisite for our transfer method to succeed is that the source and target task must be related or similar to some degree. Transfer learning simply would not work on a pair of arbitrary tasks. In the context of FACL, this means there is at least one common state variable and its MFs are shared between the source and target task. As described in Equations (4.3) and (4.4), we map the consequent parameters of the fuzzy rules in the target task to the consequent parameters of the most similar fuzzy rules in the source tasks. The similarity between the rules are measured by their degree of differences in the linguistic variables in fuzzy if-then rules. The more linguistic variables are in common, the more similar these rules are. To illustrate, we 
provide an example with a few arbitrary fuzzy rules in (4.5).

$$
\begin{aligned}
& \text { Source task: } \\
& R_{1}: \text { IF } x_{1} \text { is far AND } x_{2} \text { is far THEN } z_{1}=k_{1} \\
& \text { Target task: } \\
& R_{2}: \text { IF } x_{1} \text { is far AND } x_{2} \text { is far AND } x_{3} \text { is far THEN } z_{2}=k_{2} \\
& R_{3}: \text { IF } x_{1} \text { is far AND } x_{2} \text { is close AND } x_{3} \text { is far THEN } z_{3}=k_{3}
\end{aligned}
$$

The $x_{1}, x_{2}$ and $x_{3}$ in these fuzzy rules are linguistic variables characterized by two fuzzy sets "close" and "far". Fuzzy rule $R_{1}$ is from the source task and fuzzy rule $R_{2}$ and $R_{3}$ are from the target tasks. Using our similarity measurement, $R_{1}$ is more similar to $R_{2}$ than it is to $R_{3}$ due to more linguistic variables in common between them.

\subsubsection{Fuzzy Rule Transfer Example}

Consider a simplified scenario of modeling a self-driving agent. In the source task, we train the agent to avoid hitting pedestrians. In this case we only consider two pedestrians and thus have two state variables; the distances between the agent and two pedestrians. Things become a little more complex in the target task. We want to train the agent to not only avoid hitting the pedestrians but also reach a certain destination. In such a way, a third state variable is introduced. This scenario is illustrated in the following Table 4.1 and Table 4.2

In this example, we use two MFs for each input. Recall the rule form of a zero order Takagi-Sugeno-Kang (TSK) FIS (4.1), the fuzzy rule space in the source tasks 
Table 4.1: State variable in the source task

\begin{tabular}{|c|c|}
\hline \multicolumn{2}{|c|}{ Source Task } \\
\hline State Variable & Description \\
\hline$s_{1}$ & distance between agent and pedestrian 1 \\
\hline$s_{2}$ & distance between agent and pedestrian 2 \\
\hline
\end{tabular}

Table 4.2: State variable in the target task

\begin{tabular}{|c|c|}
\hline \multicolumn{2}{|c|}{ Target Task } \\
\hline State Variable & Description \\
\hline$s_{1}$ & distance between agent and pedestrian 1 \\
\hline$s_{2}$ & distance between agent and pedestrian 2 \\
\hline$s_{3}$ & distance between agent and destination \\
\hline
\end{tabular}

are shown as follow (4.6):

$$
\begin{aligned}
& R_{1}: \text { IF } s_{1} \text { is } A_{1} \text { and } s_{2} \text { is } B_{1} \text { Then } z_{1}=k_{1}^{\text {source }} \\
& R_{2}: \text { IF } s_{1} \text { is } A_{2} \text { and } s_{2} \text { is } B_{1} \text { Then } z_{2}=k_{2}^{\text {source }} \\
& R_{3}: \text { IF } s_{1} \text { is } A_{1} \text { and } s_{2} \text { is } B_{2} \text { Then } z_{3}=k_{3}^{\text {source }} \\
& R_{4}: \text { IF } s_{1} \text { is } A_{2} \text { and } s_{2} \text { is } B_{2} \text { Then } z_{4}=k_{4}^{\text {source }}
\end{aligned}
$$

Where $A_{k}, B_{k}$ are the fuzzy sets defined with the MFs for state variable $s_{1}$ and $s_{2}$ respectively. Similarly in the target task, we introduce a new state variable $s_{3}$ with 
two MFs. The fuzzy rule space in the target task is (4.7):

$$
\begin{aligned}
& R_{1}: \text { IF } s_{1} \text { is } A_{1} \text {, and } s_{2} \text { is } B_{1} \text { and } s_{3} \text { is } C_{1} \text { Then } z_{1}=k_{1}^{\text {target }} \\
& R_{2}: I F s_{1} \text { is } A_{2} \text {, and } s_{2} \text { is } B_{1} \text { and } s_{3} \text { is } C_{1} \text { Then } z_{2}=k_{2}^{\text {target }} \\
& R_{3}: I F s_{1} \text { is } A_{1} \text {, and } s_{2} \text { is } B_{2} \text { and } s_{3} \text { is } C_{1} \text { Then } z_{3}=k_{3}^{\text {target }} \\
& R_{4}: I F s_{1} \text { is } A_{2} \text {, and } s_{2} \text { is } B_{2} \text { and } s_{3} \text { is } C_{1} \text { Then } z_{4}=k_{4}^{\text {target }} \\
& R_{5}: I F s_{1} \text { is } A_{1} \text {, and } s_{2} \text { is } B_{1} \text { and } s_{3} \text { is } C_{2} \text { Then } z_{5}=k_{5}^{\text {target }} \\
& R_{6}: I F s_{1} \text { is } A_{2} \text {, and } s_{2} \text { is } B_{1} \text { and } s_{3} \text { is } C_{2} \text { Then } z_{6}=k_{6}^{\text {target }} \\
& R_{7}: I F s_{1} \text { is } A_{1} \text {, and } s_{2} \text { is } B_{2} \text { and } s_{3} \text { is } C_{2} \text { Then } z_{7}=k_{7}^{\text {target }} \\
& R_{8}: I F s_{1} \text { is } A_{2} \text {, and } s_{2} \text { is } B_{2} \text { and } s_{3} \text { is } C_{2} \text { Then } z_{8}=k_{8}^{\text {target }}
\end{aligned}
$$

We can immediately observe the similarity of the rules between the source and target tasks. Although the new fuzzy rule space is augmented by the additional state variables, it is still constructed upon the existing rules in the source tasks. Thus the learned knowledge can be transferred. The mapping is shown in Table 4.3 and the proposed algorithm is given in Algorithm 4.1.

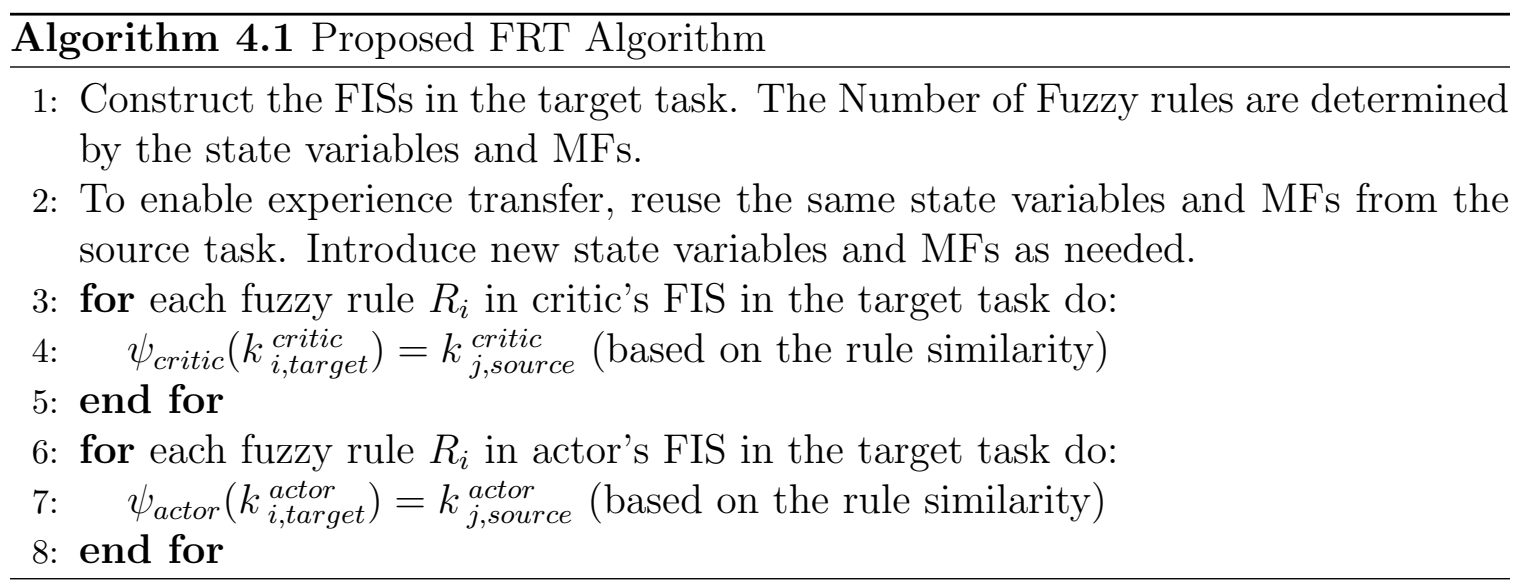


Table 4.3: Mapping of the consequent parameters in FRT

\begin{tabular}{|c|c|}
\hline \multicolumn{2}{|c|}{ Consequent Parameters } \\
\hline Target Task & Source Task \\
\hline$k_{1}^{\text {target }}$ & $k_{1}^{\text {source }}$ \\
\hline$k_{2}^{\text {target }}$ & $k_{2}^{\text {source }}$ \\
\hline$k_{3}^{\text {target }}$ & $k_{3}^{\text {source }}$ \\
\hline$k_{4}^{\text {target }}$ & $k_{4}^{\text {source }}$ \\
\hline$k_{5}^{\text {target }}$ & $k_{1}^{\text {source }}$ \\
\hline$k_{6}^{\text {target }}$ & $k_{2}^{\text {source }}$ \\
\hline$k_{7}^{\text {target }}$ & $k_{3}^{\text {source }}$ \\
\hline$k_{8}^{\text {target }}$ & $k_{4}^{\text {source }}$ \\
\hline
\end{tabular}

\subsection{Simulation and Result}

In this section, we evaluate the proposed algorithm in two sets of differential games; the pursuit-evasion game and the guarding-a-territory game. All simulation codes are written in Python from scratch since there is not an open source library available for the FACL algorithm and its simulation environment as of the date of this thesis. In the pursuit-evasion game, the evader is trying to find the fastest way to escape whereas the pursuer is trying to capture the evader. We simulate the pursuit-evasion game in two scenarios: single-agent learning and multi-agent learning. In the singleagent learning scenario, only the evader is learning while the pursuer is following a simple control strategy, which always moves in the direction of line of sight to the the evader. In the multi-agent learning scenario, both the evader and pursuers are learning using FACL. In the guarding-a-territory game, we only consider the multiagent learning scenario due to the nature of the game. In this game, both guards and invader are learning to find their optimal strategies, the goal of the guard is to intercept the invader as far as possible from the territory, whereas the goal of the 
invader is to reach the territory as close as possible while avoiding capture. Similarly, the game starts with 1 invader vs. 1 guard as our source task and later extends to 1 invader vs. 2 guards as the target task.

\subsubsection{Pursuit-Evasion Game}

The pursuit-evasion game is a differential game, and in our case, played by a high speed evader and multiple low speed pursuers. The pursuers' objective is to capture the evader, whereas the evader's objective is to escape from the pursuer as fast as possible. We start letting FACL agents play the game in a 1 evader vs. 1 pursuer setting as the source task, then extend the game to a 1 evader vs. 3 pursuers setting later in the target task. The agent kinematics are defined as (4.8),

$$
\begin{aligned}
& \dot{x}=v \cos (\theta) \\
& \dot{y}=v \sin (\theta) \\
& \dot{\theta}=\omega
\end{aligned}
$$

where $v$ represents the agent speed, $\omega$ is the angular speed of the agent, $\theta$ is the orientation of the agent with respect to $x$ axis, $x$ and $y$ are the position of the agent on the global $x$ and $y$ axis. In the design of our FACL agent, $\omega$ is the output of the actor's FIS. The kinematics equations of the players given above are solved in simulation using the second-order Runge-Kutta method.

\subsubsection{Single-agent in Pursuit-Evasion Game}

In the single-agent model, the pursuer is using a simple control strategy which always moves in the direction of the evader, while the evader is learning its optimal strategy 
using the FACL algorithm. We first start our training in the 1 evader vs. 1 pursuer scenario as our source task. The speed of pursuer is set at 1 unit/second and the evader is set at 1.2 units/second. Thus, the evader should always be able to escape. The game is lost when the evader is captured and won when the euclidean distance between the evader and pursuers becomes larger than 15 units. This means the evader has escaped from the pursuer. We use a shaping reward function for the high speed evader and it has two components, given as (4.13),

$$
R_{t+1}=d_{e p}(t+1)-d_{e p}(t)-p
$$

where $d_{e p}$ is the Euclidean distance between evader and pursuer. The component $d_{e p}(t+1)-d_{e p}(t)$ provides a positive reward if the evader moves farther from pursuer and a negative reward otherwise. The other component $p$ is a constant and it appends a penalty to the reward at every time step and pushes the agent to escape as soon as possible. When the penalty $p$ is used the evader agent escapes in a straight line away from the pursuer, as shown in Fig. 4.1. However, when the penalty $p$ is not used, the agent is still able to escape but not in a straight line, as shown in Fig. 4.2. The value of the constant $p$ is chosen based on the grid search between 0 and 1 and in this case we found the evader agent performs the best when $p$ is set to 0.2 .

There are three input state variables used in the FISs in the source task, as given in Table 4.4. We make use of triangular membership functions to define all the fuzzy sets in the various state variables. Ten symmetrical and uniformly spread triangular membership functions were defined for the heading angle of the agent $\theta_{h d}$, over the interval of $[-\pi, \pi]$. Meanwhile, seven triangular membership functions were defined for each the Manhattan distance variable $d$, over the interval of $[-40,40]$. We set the initial learning rates of the actor and the critic in the FACL algorithm as follows: 
Table 4.4: Agents' state variables in 1 evader vs. 1 pursuer

\begin{tabular}{|c|c|c|}
\hline \multicolumn{2}{|c|}{ Source Task : 1 evader vs. 1 pursuer } \\
\hline State Variable & Description & MFs and Range \\
\hline$d_{e p}^{x}$ & $\begin{array}{c}\text { manhattan distance w.r.t. x-axis } \\
\text { between evader and pursuer }\end{array}$ & $\begin{array}{c}7 \text { uniform triangular MFs over } \\
\text { the range of }[-40,40]\end{array}$ \\
\hline$d_{e p}^{y}$ & $\begin{array}{c}\text { manhattan distance w.r.t. y-axis } \\
\text { between evader and pursuer }\end{array}$ & $\begin{array}{c}7 \text { uniform triangular MFs over } \\
\text { the range of [-40, 40] }\end{array}$ \\
\hline$\theta_{h d}$ & heading angle of the agent w.r.t. x- axis & $\begin{array}{c}10 \text { uniform triangular MFs over } \\
\text { the range of }[-\pi, \pi]\end{array}$ \\
\hline
\end{tabular}

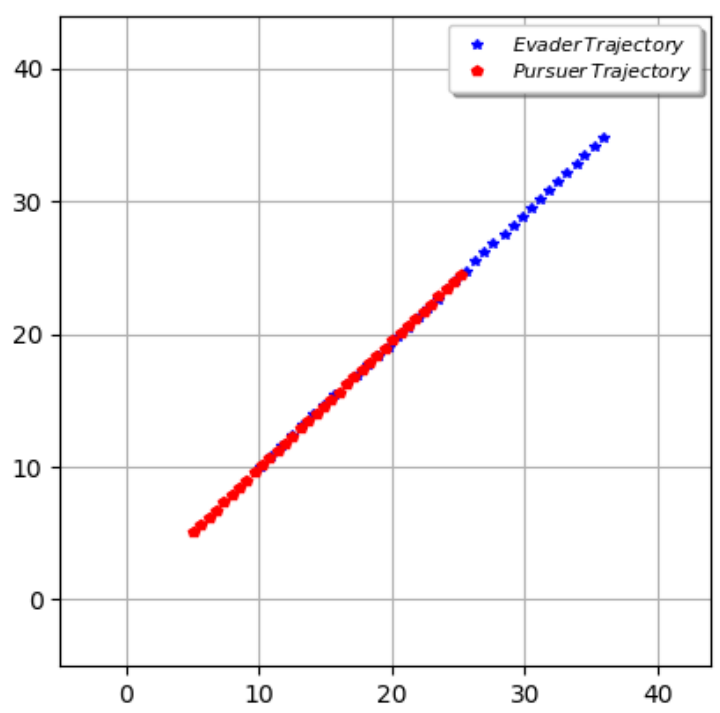

Figure 4.1: Game trajectory 1 evader vs. 1 pursuer with the time penalty constant and the evader escapes

$\beta_{0}=0.05$ and $\alpha_{0}=0.1$. We set $\beta_{0}<\alpha_{0}$ so that the actor will converge slower than critic to prevent instability in the actor [20]. The variance of the initial exploration noise $\sigma$ and the discount factor $\gamma$ are set as follows: $\sigma=3 \mathrm{rad} /$ second and $\gamma=0.25$. The decay rates of the learning factors and exploration noise after each epoch $\mathrm{k}$ are set as follows: $\beta_{k+1}=0.995^{k} \beta_{0}, \alpha_{k+1}=0.999^{k} \alpha_{0}$, and $\sigma_{k+1}=0.994^{k} \sigma_{0}$. The values for the learning rates, the exploration noise and the discount factors were chosen 


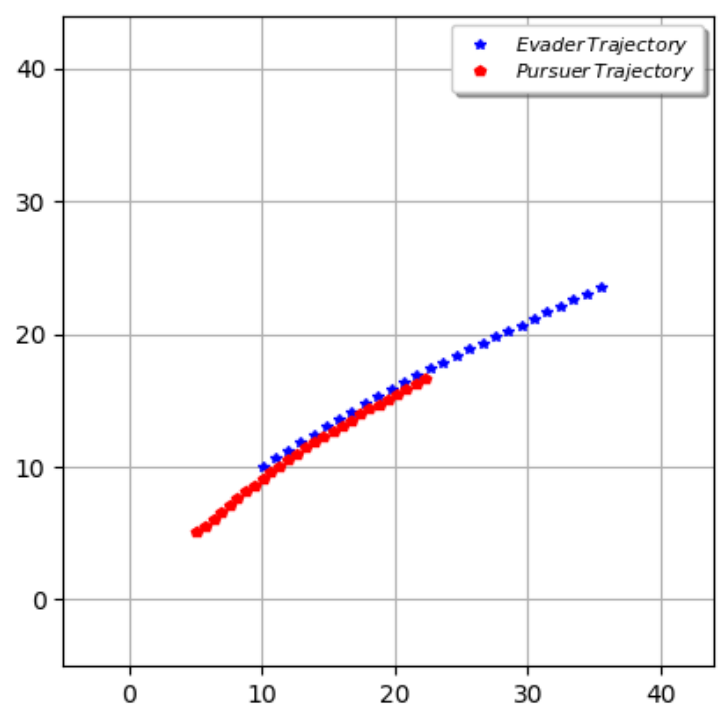

Figure 4.2: Game trajectory 1 evader vs. 1 pursuer without the time penalty constant and the evader escapes

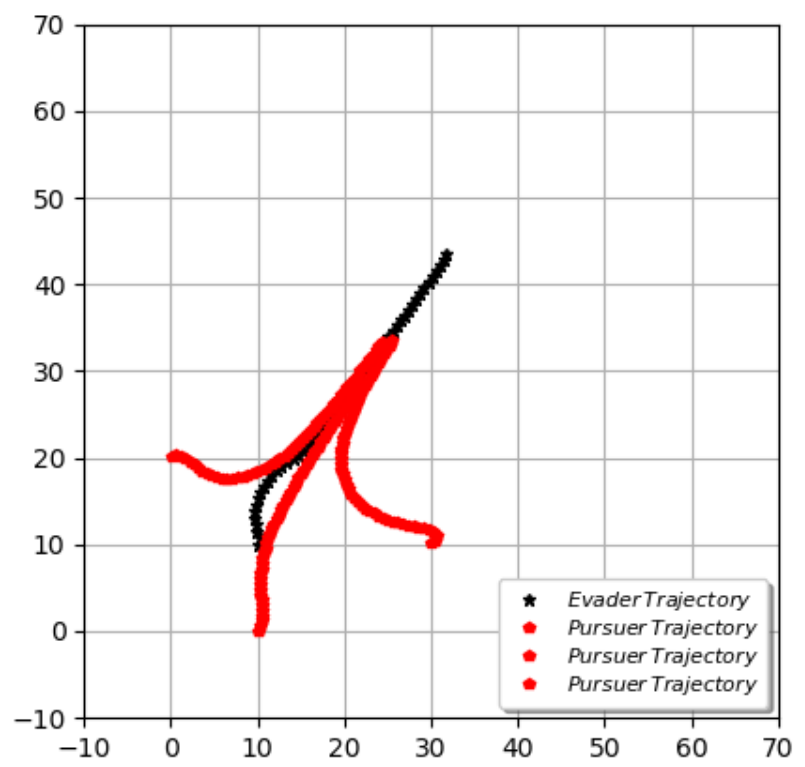

Figure 4.3: Game trajectory 1 evader vs. 3 pursuer 
based on a priori knowledge of the problem and heuristics in experiments. These configurations are proven to be working in our simulation. To achieve an appropriate comparison when evaluating the fuzzy rule transfer, we use the same configurations as above in the target task throughout the simulation.

We first let the single pursuer and evader play the game for 1000 epochs and the final game trajectory is shown in Fig. 4.1. This result shows that the evader agent learned the fastest way to escape, namely, moving as a straight line in the opposite direction of the pursuer. For the target task, we let our agent play a similar game that is closely related to our source task. This time the three pursuers started from three different positions around the evader, as shown in Fig. 4.3. All three pursuer agents are identical and follow a simple control strategy as in the source task. However, the state space is changed for the evader due to the additional pursuers. Instead of having three state variables, in the target task we now have seven state variables implemented in the FISs. The list of state variables in the target task is presented in the Table 4.5.

Meanwhile, our reward function is also expanded to account for the distance between the evader and the additional pursuers, given as (4.10).

$$
\begin{aligned}
R_{t+1}= & d_{e p 1}(t+1)-d_{e p 1}(t)+d_{e p 2}(t+1) \\
& -d_{e p 2}(t)+d_{e p 3}(t+1)-d_{e p 3}(t)-p
\end{aligned}
$$

Where $d_{e p 1}$ is the Euclidean distance between the evader and pursuer $1, d_{e p 2}$ is the Euclidean distance between the evader and pursuer 2 and so forth. Similar to the 1 evader vs. 1 pursuer case, we introduce a constant $\mathrm{p}$ in the reward function in order to push the evader agent to escape as soon as possible. As the number of pursuers has 
Table 4.5: Evader's state variables in 1 evader vs. 3 pursuer

\begin{tabular}{|c|c|c|}
\hline \multicolumn{3}{|c|}{ Target Task : 1 evader vs. 3 pursuer } \\
\hline State Variable & Description & MFs and Range \\
\hline$d_{e p 1}^{x}$ & $\begin{array}{l}\text { manhattan distance w.r.t. } \mathrm{x} \text {-axis } \\
\text { between evader and pursuer } 1\end{array}$ & $\begin{array}{l}7 \text { uniform triangular MFs over } \\
\text { the range of }[-40,40]\end{array}$ \\
\hline$d_{e p 1}^{y}$ & $\begin{array}{l}\text { manhattan distance w.r.t. y-axis } \\
\text { between evader and pursuer } 1\end{array}$ & $\begin{array}{c}7 \text { uniform triangular MFs over } \\
\text { the range of }[-40,40]\end{array}$ \\
\hline$d_{e p 2}^{x}$ & $\begin{array}{l}\text { manhattan distance w.r.t. } \mathrm{x} \text {-axis } \\
\text { between evader and pursuer } 2\end{array}$ & $\begin{array}{l}7 \text { uniform triangular MFs over } \\
\text { the range of }[-40,40]\end{array}$ \\
\hline$d_{e p 2}^{y}$ & $\begin{array}{l}\text { manhattan distance w.r.t. } y \text {-axis } \\
\text { between evader and pursuer } 2\end{array}$ & $\begin{array}{l}7 \text { uniform triangular MFs over } \\
\text { the range of }[-40,40]\end{array}$ \\
\hline$d_{e p 3}^{x}$ & $\begin{array}{l}\text { manhattan distance w.r.t. } \mathrm{x} \text {-axis } \\
\text { between evader and pursuer } 3\end{array}$ & $\begin{array}{l}7 \text { uniform triangular MFs over } \\
\text { the range of }[-40,40]\end{array}$ \\
\hline$d_{e p 3}^{y}$ & $\begin{array}{c}\text { manhattan distance w.r.t. y-axis } \\
\text { between evader and pursuer } 3\end{array}$ & $\begin{array}{l}7 \text { uniform triangular MFs over } \\
\text { the range of }[-40,40]\end{array}$ \\
\hline$\theta_{h d}$ & heading angle of the agent w.r.t. $x$ - axis & $\begin{array}{l}10 \text { uniform triangular MFs over } \\
\text { the range of }[-\pi, \pi]\end{array}$ \\
\hline
\end{tabular}

tripled in the target task, we increase the p to 0.6 and we observe good performance after trials.

Furthermore, because of the increased number of state variables in the target task, the fuzzy rule space has increased to 1176490 rules instead of the 490 rules in the source task. The total number of rules are calculated based on the number of MFs and state variables as shown in (4.2). Although the rule space in the target task is extremely large, because we use triangular membership functions in our design, at any time step only $2^{n}$ rules would fire, where $n$ is the number of inputs [19]. Thus, we only need to identify the rule sets that fire based on the input state variables then calculate the firing strength for the rules in the rule sets. Therefore, this process is computationally achievable. However, in this case since there are 7 input state variables, we need to compute 256 rules in total for both the critic and actor in FACL 
at any given time step. It is still quite an expensive computational process and the agent may not converge to a good policy within a short time. Nevertheless, if we use a different type of membership function such as Gaussian membership function, the computational complexity will increase dramatically, although the transfer process will be the same since the number of the fuzzy rules and consequent parameters does not change.

We perform the fuzzy rule transfer in the FACL agent in the target task as described in Section 4.1 and Algorithm 4.1. The mapping of the fuzzy rules are performed both on critic and actor of the FACL agent, as presented in (4.3) and (4.4), through which we assign the initial value of the consequent parameters in the target task based on the learned consequent parameters in the source task and similarity of the fuzzy rules. In our simulation software, this can be achieved with a for loop that iterates through the fuzzy rules space in the target task to assign weights to the consequent parameters. This process needs only be completed once in the beginning of the simulation in the target task. To promote better understanding, we show one example of how the mapping and assignment is done for a fuzzy rule below. Suppose we have a fuzzy rule $R_{m}$ in the target task,

$$
\begin{aligned}
& R_{m}: I F d_{e p 1}^{x} \text { is } M F_{d_{e p 1}^{x}}^{1} \text { and } d_{e p 1}^{y} \text { is } M F_{d_{e p 1}^{y}}^{1} \text { and } d_{e p 2}^{x} \text { is } M F_{d_{e p 2}^{x}}^{1} \text { and } d_{e p 2}^{y} \text { is } M F_{d_{e p 2}^{y}}^{1} \\
& \quad \text { and } d_{e p 3}^{x} \text { is } M F_{d_{e p 3}^{x}}^{1} \text { and } d_{e p 3}^{y} \text { is } M F_{d_{e p 3}^{y}}^{1} \text { and } \theta_{h d} \text { is } M F_{\theta_{h d}}^{1} \text { Then } z_{m}=k_{m}^{\text {target }}
\end{aligned}
$$

where $d_{e p 1}^{x}$ to $\theta_{h d}$ are the input state variables presented in Table 4.5. $M F_{d_{e p 1}^{x}}^{1}$ is the first membership function of the state variable $d_{e p 1}^{x}, M F_{d_{e p 1}^{y}}^{1}$ is the first membership function of the state variable $d_{e p 1}^{y}$, and so on. Based on the similarity in the fuzzy 


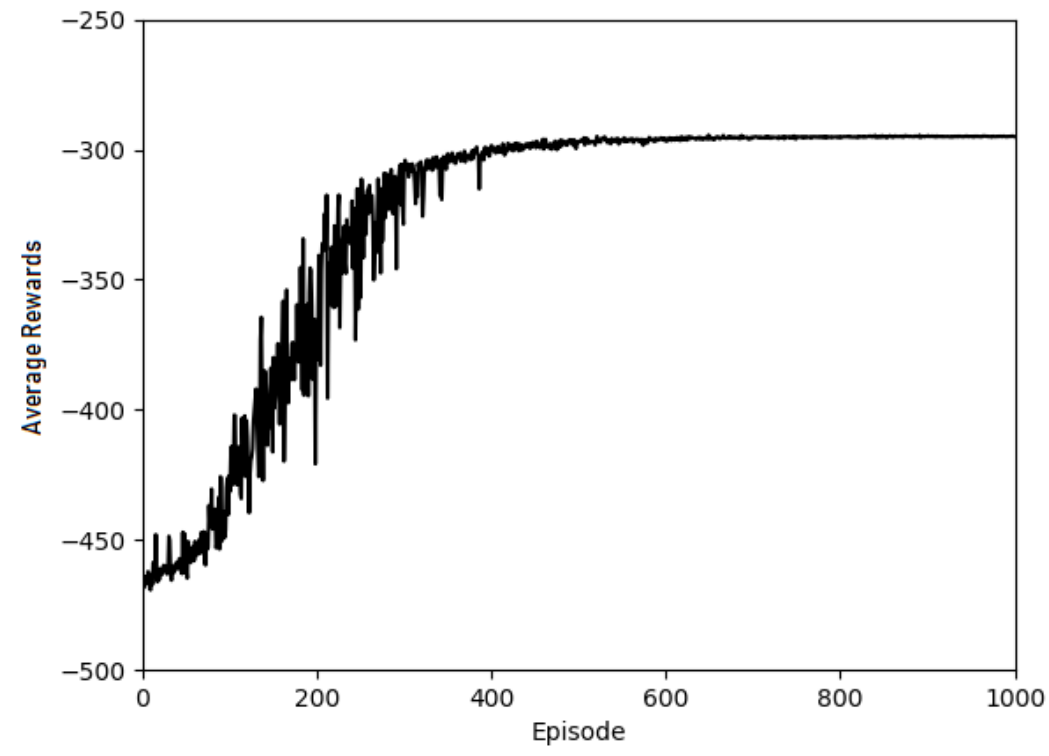

Figure 4.4: Average reward using FRT in 1 evader vs. 3 pursuers game (only the evader is learning)

rule, we map this rule to the most similar rule $R_{n}$ in the source task,

$$
R_{n}: I F d_{e p 1}^{x} \text { is } M F_{d_{e p 1}^{x}}^{1} \text { and } d_{e p 1}^{y} \text { is } M F_{d_{e p 1}^{y}}^{1} \text { and } \theta_{h d} \text { is } M F_{\theta_{h d}}^{1} \text { Then } z_{n}=k_{n}^{\text {source }}
$$

where $k_{n}^{\text {source }}$ is the learned value of the consequent parameter in the source task. Thus, we assign the value in the target task: $z_{m}=k_{m}^{\text {target }}=k_{n}^{\text {source }}$.

For our evaluation, we let the agents play the same pursuit-evasion game (1 evader vs. 3 pursuers) with and without the FRT in place. The simulation is run for 1000 epochs and in both situations, the agents have learned and converged to the same policy which leads to the escape from the three pursuers, as shown in the game plot Fig. 4.3. However, with the FRT in place, the agent learns significantly faster. We run our simulation with 10 trials, with 1000 epochs per trial. The accumulated reward 


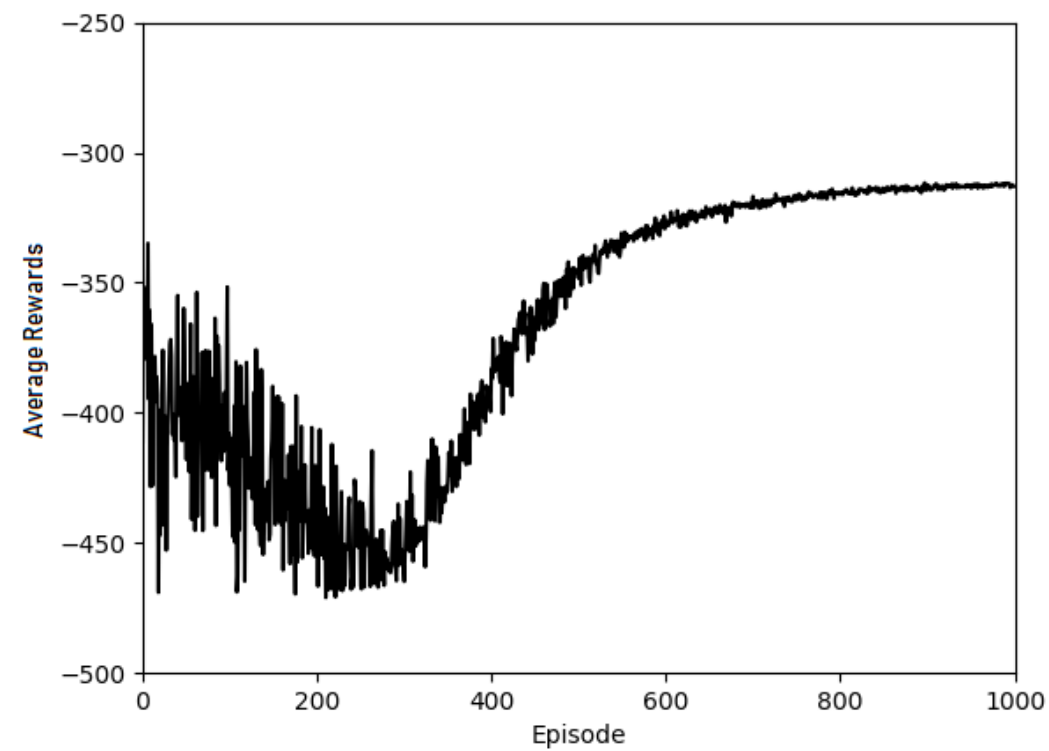

Figure 4.5: Average reward without Transfer in 1 evader vs. 3 pursuers game (only the evader is learning)

collected by the agent, averaged over 10 learning trials for both learning with and without transfer are shown in Fig. 4.4 and Fig. 4.5 respectively.

As illustrated in the figures, the convergence speed of the reward trajectory in the FRT case is much faster. This indicates that the agent is able to learn faster when using FRT. In addition, we present the movement trajectory of the agents at 500 epochs in both simulations with and without FRT in Fig. 4.6 and Fig. 4.7 respectively for comparison. In Fig. 4.6, the evader agent has learned a good policy after approximately 500 epochs of training, and successfully escapes after approximately 350 time steps in that episode. Whereas in Fig. 4.7, the agent still struggles to achieve a good policy after approximately the same number of epochs into the training. The escape of the evader agent takes place around 400 time steps in that episode. 


\subsubsection{Multi-agent Learning in Pursuit-Evasion Game}

This section present the case for the multi-agent learning scenario where all agents are learning. The simulation environment is set up nearly identical to the 1 evader vs. 3 pursuers game in the single-agent learning scenario presented earlier, except this time the pursuers are learning and located with different initial positions such that the evader is able to be captured by the pursuers. The configuration of the game, including the values for the learning rates, the exploration noise and the discount factor in FACL agents, is identical to the single-agent learning scenario presented in Section 4.2.1.1. The design of the state variables in a pursuer agent is identical to the evader agent in the 1 evader vs. 1 pursuer case, as presented in Table 4.4. The reward function of the pursuer is defined as:

$$
R_{t+1}=d_{e p}(t)-d_{e p}(t+1)
$$

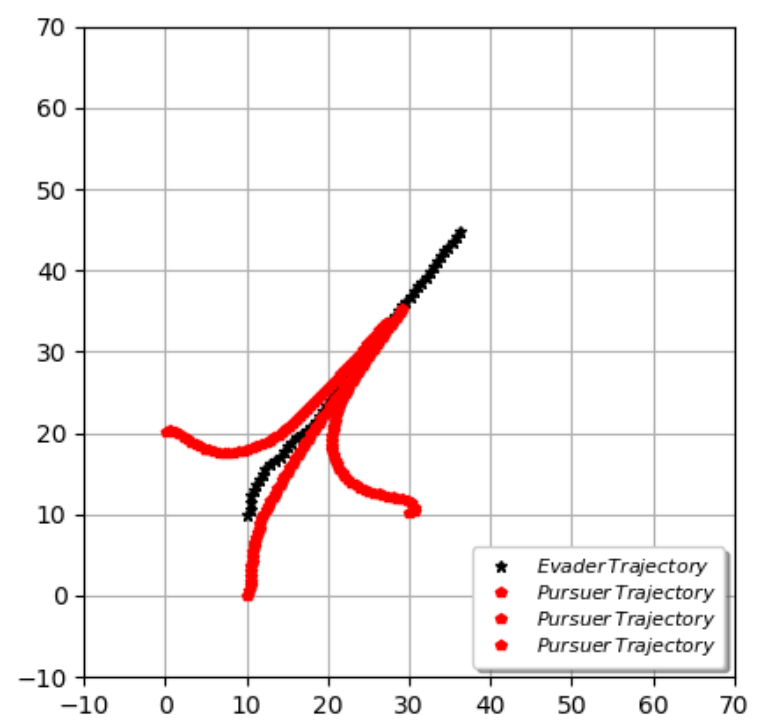

Figure 4.6: Epoch 500 using FRT in 1 evader vs. 3 pursuers game (only the evader is learning) 


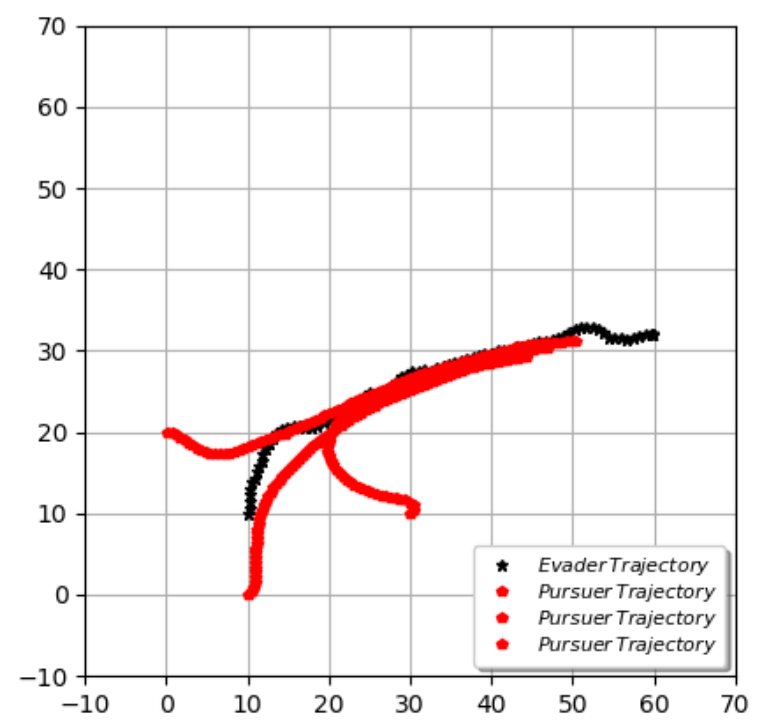

Figure 4.7: Epoch 500 without FRT in 1 evader vs. 3 pursuers game (only the evader is learning)

Where $d_{e p}$ is the Euclidean distance between the evader and the pursuer. The reward $d_{e p}(t)-d_{e p}(t+1)$ becomes positive if the pursuer moves closer to the evader and a negative reward otherwise. Unlike the evader's reward function, we do not use a penalty constant since it is not required for the pursuers to capture the evader as quickly as possible but rather to capture them at all. For the source task, we first set up the FACL agents to play a 1 evader vs. 1 pursuer game for 1000 epochs. The final game trajectory is identical to Fig. 4.1. Both the evader agent and pursuer agents have learned their best strategies to escape or pursue.

Now we move to the target task where the evader tries to escape from the three pursuers and all agents are learning using the FACL algorithm. In this scenario, the initial positions of the agents are changed such that the pursuers are able to capture the evader. In order to explain the initial capture condition, we present an example here. Fig. 4.8 illustrates the initial condition of the three pursuers labeled 


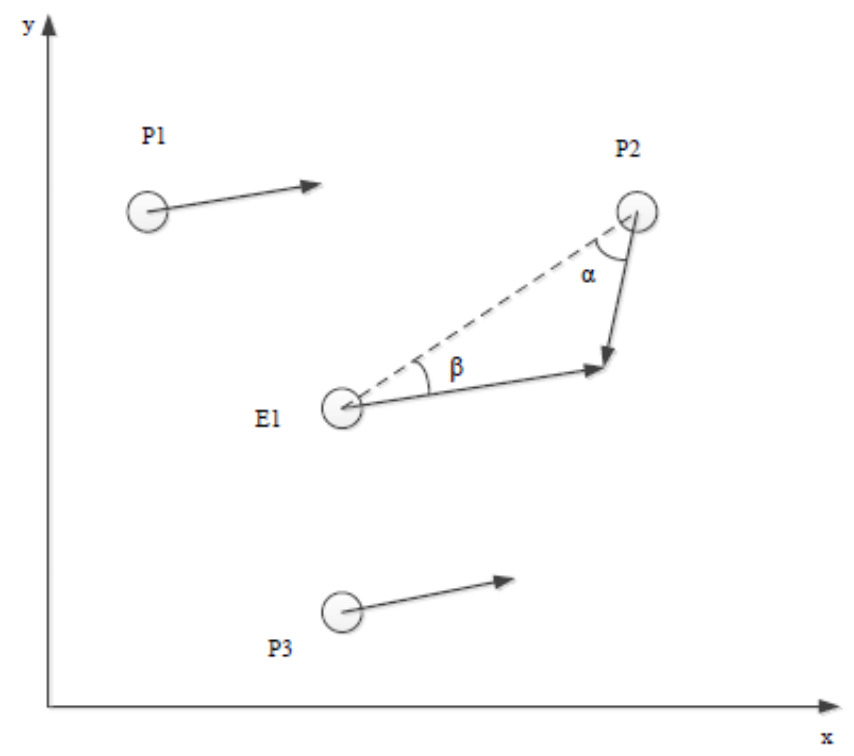

Figure 4.8: High speed evader E1 chased by three pursuers, P1, P2 and P3

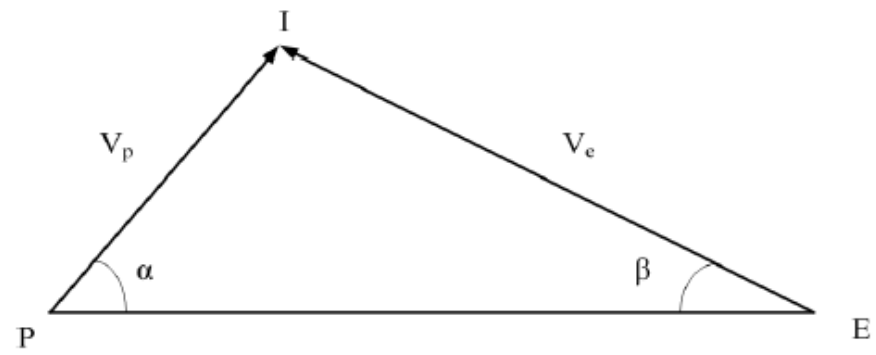

Figure 4.9: Geometric illustration of capture condition

as P1, P2, and P3 and the high speed evader labeled as E1. Each pursuer has the same constant speed $V_{p}$. The evader also has a constant speed $V_{e}$, where $V_{e}>V_{p}$. The positions of the pursuers and the evader are $\left(x_{p_{i}}(t), y_{p_{i}}(t)\right)$ and $\left(x_{e}(t), y_{e}(t)\right)$, respectively. The capture of the superior evader occurs if the distance between the pursuer $P_{i}$ and the evader $E$ is less than or equal to the capture distance $d_{c}$, such that, $\left\|\left(x_{p_{i}}, y_{p_{i}}\right)-\left(x_{e}, y_{e}\right)\right\| \leq d_{c}$. Fig. 4.9 shows the condition required for the pursuer $P$ to capture the evader $E$ at the point $I$. Fig. 4.9 illustrates the triangle property that defines the capture condition [19], 


$$
\frac{\sin \alpha}{\sin \beta}=\frac{V_{e}}{V_{p}}
$$

where the angle $\beta$ is the angle difference between the direction of the evader and the direction of the evader's line of sight (LOS) to the pursuer $P$, and the angle $\alpha$ is the angle difference between the direction of the pursuer $P$ and the direction of the pursuer's LOS to the evader $E$. The pursuer's goal or objective is to select a heading angle $\alpha$, such that the pursuer $P$ can capture the higher speed evader, $E$. If there exists an angle $\alpha$, in the range [0, $\pi / 2]$, computed as [19],

$$
\alpha=\arcsin \left(\frac{V_{e}}{V_{p}} \sin \beta\right)
$$

then pursuer $P$ can capture evader $E$. When evader $E$, moves into a region where pursuer $P$ can select an angle $\alpha$ that satisfies the condition of (4.15), that region is called the capture region of pursuer $P[19]$. On the other hand, when the evader moves into a region where pursuer $P$ cannot select an angle $\alpha$ that satisfies the condition in (4.15), that region is called the escape region of pursuer $P$ [19].

We have already presented the FRT process for the evader in Section 4.2.1.1. Whereas for the pursuer agent, the transfer is straightforward since each of them is learning individually and they do not share knowledge with other pursuer agents. This means the state space for the pursuer agent does not change between the source and target task. Therefore, the fuzzy rule mapping is not needed and a 1-to-1 direct copy of the learned consequence parameters of each fuzzy rule of the pursuer agent is performed between the source and target task. This is a special scenario where state space is identical between the source and target task.

The original game trajectory after 600 episodes is shown in Fig. 4.10. When FRT is used, all agents have converged to new policies and the game ends much faster. 


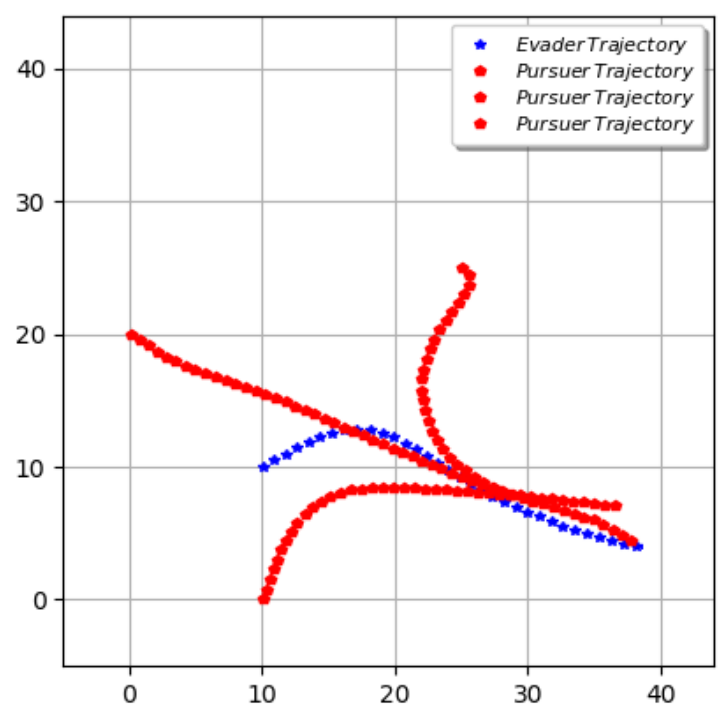

Figure 4.10: Epoch 600 without FRT in multi-agent 1 evader vs. 3 pursuers game when all agents are learning

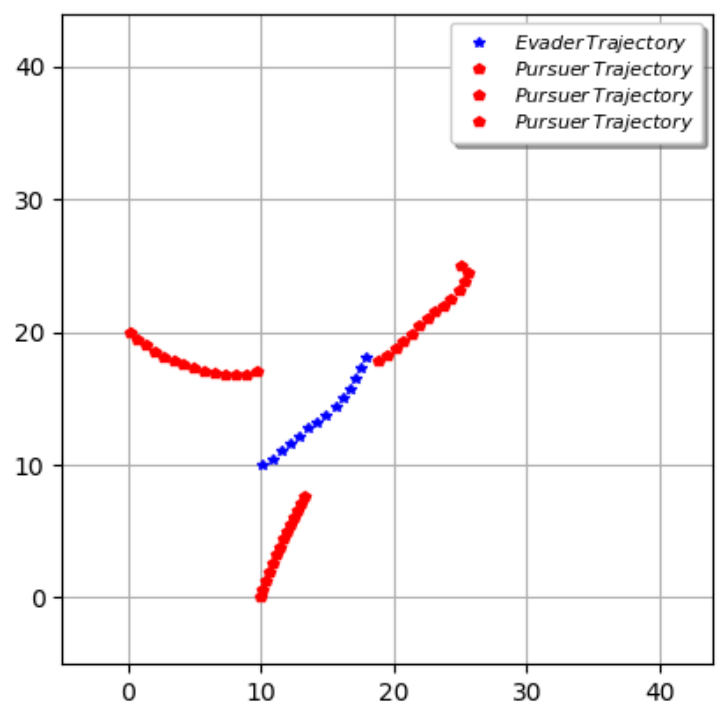

Figure 4.11: Epoch 600 with FRT in multi-agent 1 evader vs. 3 pursuers game when all agents are learning 
We present the game trajectory after 600 episodes when FRT is used in Fig. 4.11. We observe that the transfer learning benefits the pursuers more than it does for the evader as the pursuers are able to capture the evader faster. This behavior is due to the identical state space of the pursuers between the source and target task. The final performance of the pursuer after 600 episode in the target task can be seen as a result of the training after 1200 episodes, with 600 episodes from the source task and a configuration (learning rate, exploration noise and discount factors) reset before the target task begins. However, for the evader agent it still requires learning a new state space. Thus, the pursuers have an advantage over the evader when transfer learning is used. With the transferred knowledge, the FACL agents are initialized with the pre-learned consequent parameters from the source task. Thus there are greater exploration around those weights in the early stage of the game, which leads to different state evaluation and action selection for the agents. Since all agents are learning and interacting with each other, the states of the game become more dynamic compared to the single-agent learning scenario in which pursuers are merely following a static control strategy. Combining all the above factors, the FACL agents have converged to different weights in the multi-agent game.

To understand how FRT impact the convergence of the consequent parameters, we pick one arbitrary fuzzy rule from the evader and pursuer agent respectively and show their trajectories throughout the game. The trajectories of the consequent parameters for the evader agent with and without the FRT are shown in Fig. 4.12 and Fig. 4.13. In the FRT case, we observe that the weights for the fuzzy rule 420175 in both actor and critic are initialized with the pre-learned values around 12.3 and -10 respectively. Whereas in the non-FRT case, the weights are initialized with 0 . The trajectories of the consequent parameters fluctuated less and converge faster in the FRT case, particularly for the actor. Likewise, the trajectories of the consequent parameters for 
the pursuer agent with and without the FRT are shown in Fig. 4.14 and Fig. 4.15. We observe that the weights for the fuzzy rule 314 in both actor and critic are initialized around 1.5 and 0.5 respectively in the FRT case. They converge quickly from the initial points during the training. Whereas in the non-FRT case, the trajectories of the consequents parameters for the fuzzy rule 314 start with 0 . They fluctuate more and converge slower from the initial point during the training.

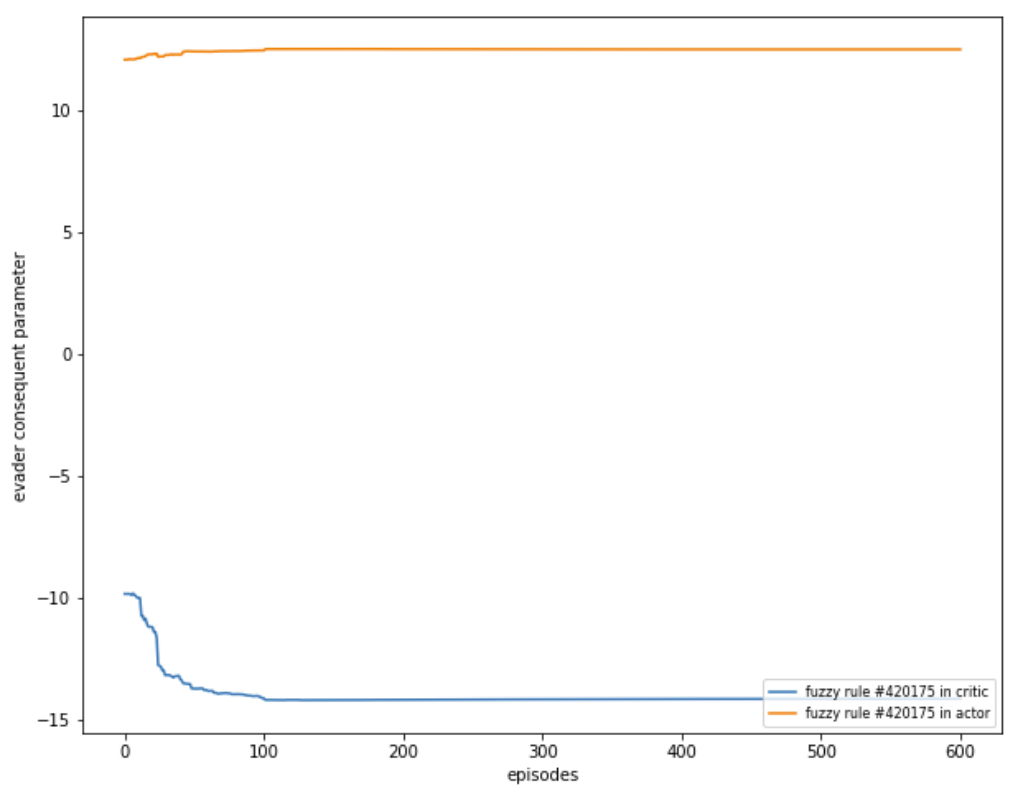

Figure 4.12: Consequent parameters for evader in 1 evader vs. 3 pursuers game when all agents are learning with FRT

\subsubsection{Guarding a Territory Game}

The guarding-a-territory differential game was introduced by Isaacs [34]. It is a zerosum differential game with continuous state space and action space. Both guards and the invader are learning with the FACL algorithm to find their optimal strategies in this game. The goal of the guard is to intercept the invader as far as possible from the territory, whereas the goal of the invader is to reach the territory as close as possible while avoiding capture. The dynamics of the FACL agents are identical 
Table 4.6: The agent's state variables in 1 invader vs. 1 guard

\begin{tabular}{|c|c|c|}
\hline \multicolumn{3}{|c|}{ Source Task : 1 invader vs. 1 guard } \\
\hline State Variable & Description & MFs and Range \\
\hline$d_{I G}^{x}$ & $\begin{array}{c}\text { manhattan distance w.r.t. x-axis } \\
\text { between invader and guard }\end{array}$ & $\begin{array}{c}7 \text { uniform triangular MFs over } \\
\text { the range of [-40,40] }\end{array}$ \\
\hline$d_{I G}^{y}$ & $\begin{array}{c}\text { manhattan distance w.r.t. y-axis } \\
\text { between invader and guard }\end{array}$ & $\begin{array}{c}7 \text { uniform triangular MFs over } \\
\text { the range of [-40,40] }\end{array}$ \\
\hline$\theta_{T}$ & $\begin{array}{c}\text { angle between the x-axis and Agent's } \\
\text { line of sight towards the territory }\end{array}$ & $\begin{array}{c}10 \text { uniform triangular MFs over } \\
\text { the range of }[-\pi, \pi]\end{array}$ \\
\hline
\end{tabular}

to the pursuit-evasion game, defined in equation (4.8). The invader is running at a higher speed than the guard. The speed of the guard is set at 1 unit/second and the invader is set at 1.3 units/second. For each episode in the training, the game terminates when the invader reaches the territory or gets caught by the guard. This game has been studied and implemented using the FACL learning algorithm in [33].

Similar to the pursuit-evasion game presented in Section 4.2.1, we use triangular

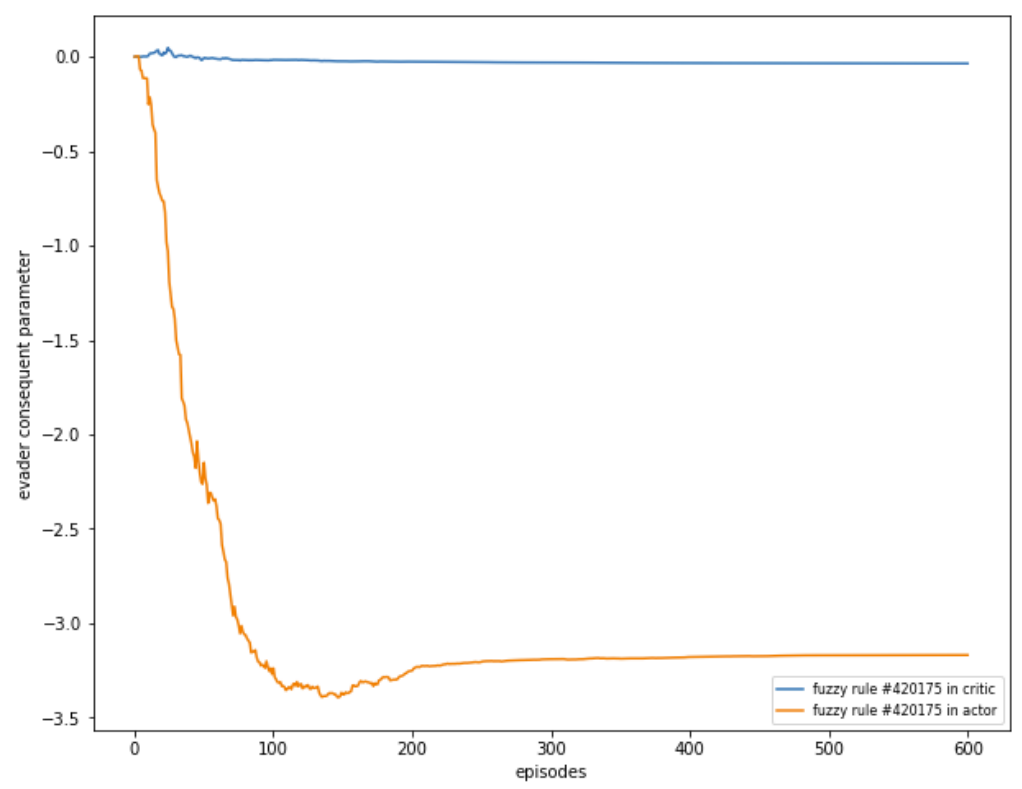

Figure 4.13: Consequent parameters for evader in 1 evader vs. 3 pursuers game when all agents are learning without FRT 


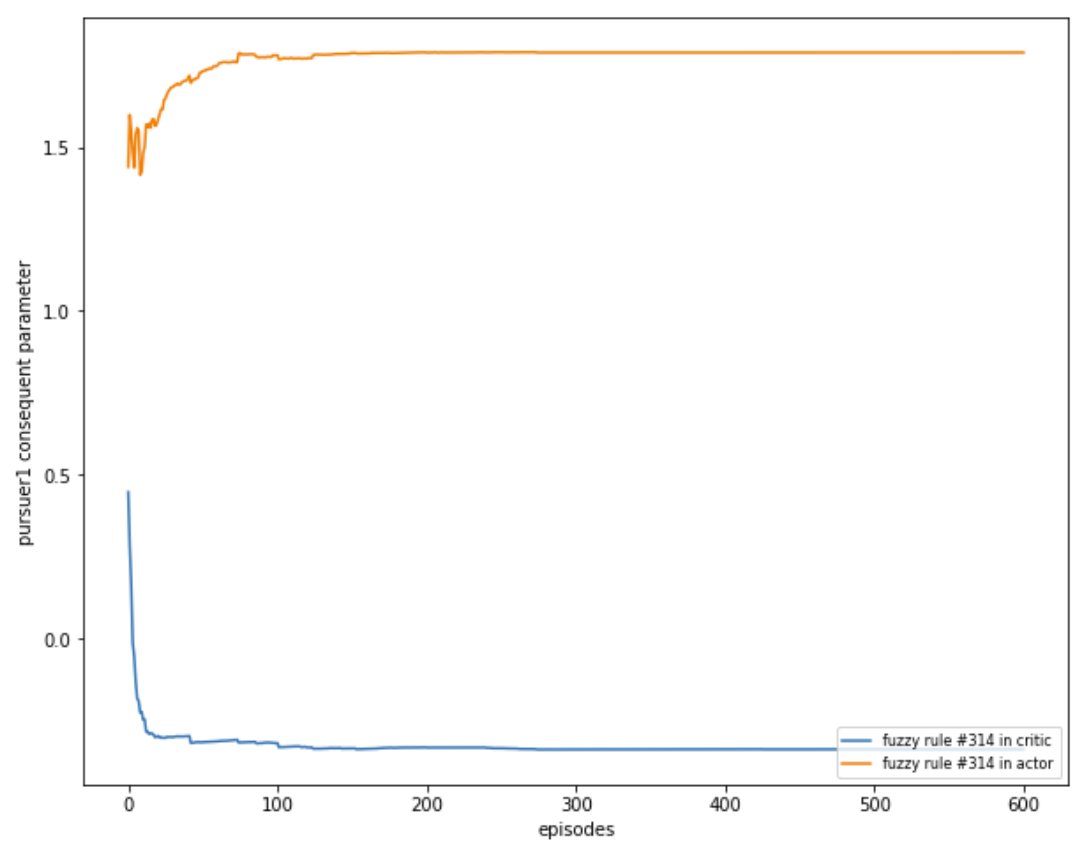

Figure 4.14: Consequent parameters for pursuer1 in 1 evader vs. 3 pursuers game when all agents are learning with FRT

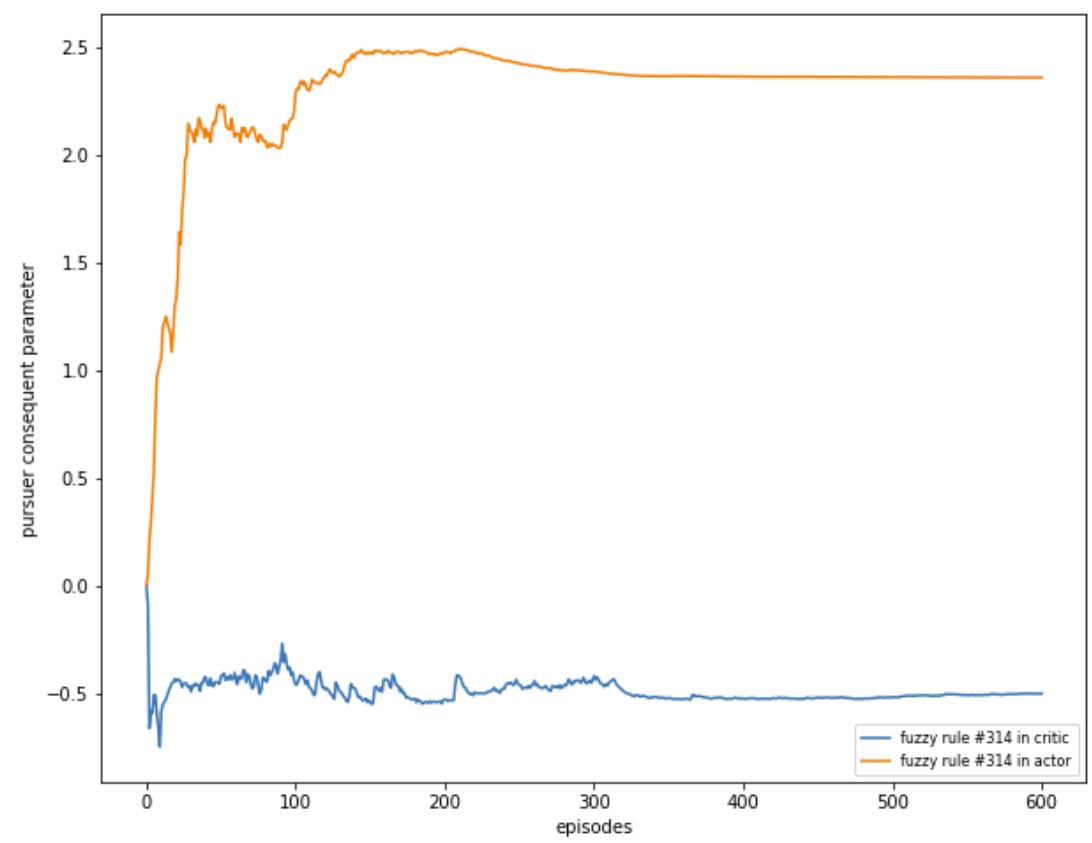

Figure 4.15: Consequent parameters for pursuer1 in 1 evader vs. 3 pursuers game when all agents are learning without FRT 
Table 4.7: Invader's state variables in 1 invader vs. 2 guards

\begin{tabular}{|c|c|c|}
\hline \multicolumn{3}{|c|}{ Target Task : 1 invader vs. 2 guards } \\
\hline State Variable & Description & MFs and Range \\
\hline$d_{I G 1}^{x}$ & $\begin{array}{c}\text { manhattan distance w.r.t. x-axis } \\
\text { between invader and guard } 1\end{array}$ & $\begin{array}{c}7 \text { uniform triangular MFs over } \\
\text { the range of [-40, 40] }\end{array}$ \\
\hline$d_{I G 1}^{y}$ & $\begin{array}{c}\text { manhattan distance w.r.t. y-axis } \\
\text { between invader and guard 1 }\end{array}$ & $\begin{array}{c}7 \text { uniform triangular MFs over } \\
\text { the range of [-40, 40] }\end{array}$ \\
\hline$d_{I G 2}^{x}$ & $\begin{array}{c}\text { manhattan distance w.r.t. x-axis } \\
\text { between invader and guard } 2\end{array}$ & $\begin{array}{c}7 \text { uniform triangular MFs over } \\
\text { the range of [-40, 40] }\end{array}$ \\
\hline$d_{I G 2}^{y}$ & $\begin{array}{c}\text { manhattan distance w.r.t. y-axis } \\
\text { between invader and guard 2 }\end{array}$ & $\begin{array}{c}7 \text { uniform triangular MFs over } \\
\text { the range of [-40, 40] }\end{array}$ \\
\hline$\theta_{T}$ & $\begin{array}{c}\text { angle between the x-axis and invader's } \\
\text { line of sight towards the territory }\end{array}$ & $\begin{array}{c}10 \text { uniform triangular MFs over } \\
\text { the range of [- } \pi, \pi]\end{array}$ \\
\hline
\end{tabular}

membership functions to define the fuzzy sets in the state variables. Ten symmetrical and uniformly spread triangular membership functions are defined for the angle $\theta_{T}$ and seven triangular membership functions are defined for each the Manhattan distance variable $d_{I G}$. The configuration inside the FACL agents are identical between the invader and guard in a 1 invader vs. 1 guard game. We show the state variables in Table 4.6. We set the initial learning rates of the actor and the critic in the FACL algorithm as follows: $\beta_{0}=0.05$ and $\alpha_{0}=0.1$. We set $\beta_{0}<\alpha_{0}$ so that the actor will converge slower than critic to prevent instability in the actor [20]. The variance of the initial exploration noise $\sigma$ and the discount factor $\gamma$ are set as follows: $\sigma=3$ $\mathrm{rad} / \mathrm{second}$ and $\gamma=0.25$. The decay rates of the learning factors and exploration noise after each epoch $\mathrm{k}$ are set as follows: $\beta_{k+1}=0.995^{k} \beta_{0}, \alpha_{k+1}=0.999^{k} \alpha_{0}$, and $\sigma_{k+1}=0.994^{k} \sigma_{0}$. The values for the learning rates, the exploration noise and the discount factor were chosen based on a priori knowledge of the problem and heuristics in experiments. Based on the previous research in [33], the reward function for the 
invader in the 1 invader vs. 1 guard case is designed as:

$$
R_{t+1}=D\left(d_{i t}(t)-d_{i t}(t+1)\right)-J\left(d_{i g}(t)-d_{i g}(t+1)\right)
$$

Where $d_{i t}$ is the Euclidean distance between the invader and territory and $d_{i g}$ is the Euclidean distance between the invader and guard. The component $D\left(d_{i t}(t)-\right.$ $\left.d_{i t}(t+1)\right)$ provides a positive reward if the invader moves closer to the territory and a negative reward otherwise. Similarly, the component $-J\left(d_{i g}(t)-d_{i g}(t+1)\right)$ provides a negative reward if the invader moves closer to the guard and a positive reward otherwise. The constant $\mathrm{D}$ and $\mathrm{J}$ determines the importance attached to the various components of the reward function. According to [33], we set $\mathrm{D}=3, \mathrm{~J}=1$ and the reward function for the guard is defined as:

$$
R_{t+1}=P\left(d_{i g}(t)-d_{i g}(t+1)\right)+M\left(d_{i t}(t)-d_{i t}(t+1)\right)
$$

Where $d_{g t}$ is the Euclidean distance between the guard and the territory and $d_{i g}$ is the Euclidean distance between the invader and the guard. Likewise, we set $\mathrm{P}=1.1$ and $\mathrm{M}=1.0$ as in [33].

We first set up the agents to play the game in a 1 invader vs. 1 guard setting. The game trajectory of the agents' movement after 600 epochs is shown in Fig. 4.16. We then simulate the target task in which an additional guard is introduced into the game. Therefore, the input state space for the invader agent becomes different in the target task. We show the design of the new state variables and MFs in Table 4.7. With the new state space, the number of the rules in the target task has increased from 490 to 24010. However for guards, the state space remains unchanged between the source and target tasks. Thus, the number of the rules for guards still remains 490. As described in the pursuit-evasion game in Section 4.2.1, we use the same FRT 
approach to perform the knowledge transfer on the invader agent while directly copy the learned consequent parameters from the source task to the target task for guards. The reward function for the guard does not change but the new reward function for invader in the target task becomes:

$$
R_{t+1}=D\left(d_{i t}(t)-d_{i t}(t+1)\right)-J 1\left(d_{i g 1}(t)-d_{i g 1}(t+1)\right)-J 2\left(d_{i g 2}(t)-d_{i g 2}(t+1)\right)
$$

where $J 2\left(d_{i g 2}(t)-d_{i g 2}(t+1)\right)$ is the new component introduced in the reward function due to the additional guard. $d_{i g 1}$ is the Euclidean distance between the invader and guard 1 and $d_{i g 2}$ is the Euclidean distance between the invader and guard 2 . We set $\mathrm{D}=3$ and $\mathrm{J} 1=1$ and $\mathrm{J} 2=1$ according to [33].

In the 1 invader vs. 2 guards target task, we let the FACL agents play the game with and without using the FRT. In both cases, all three players are able to learn and

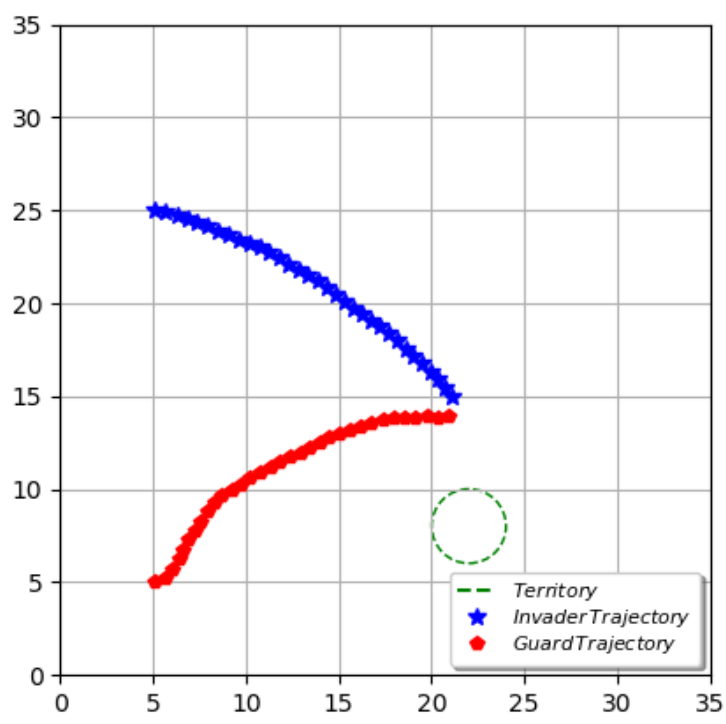

Figure 4.16: Game trajectory 1 invader vs. 1 guard in Territory game as the source task 


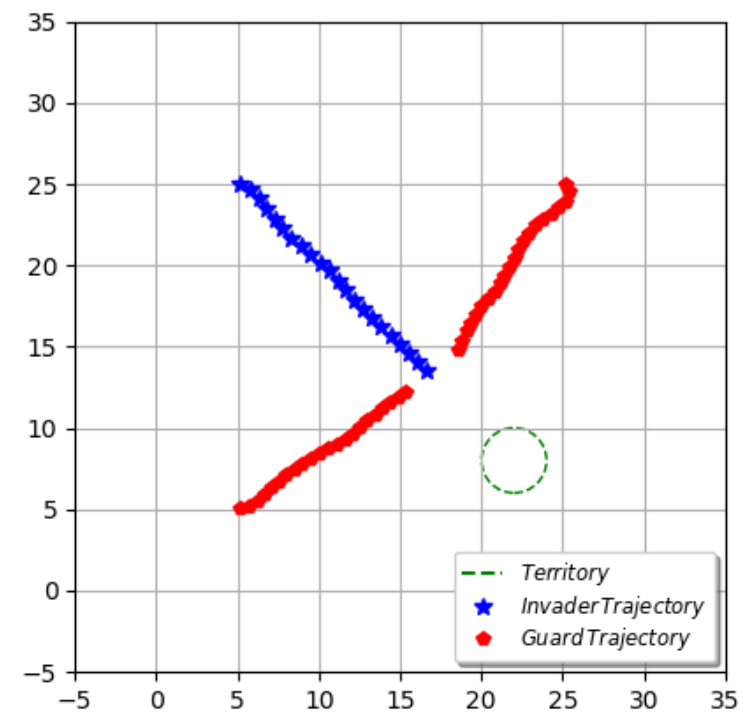

Figure 4.17: Game trajectory 1 invader vs. 2 guard in Territory game as the target task

converge to their optimal strategies, as shown in Fig. 4.17. The optimal strategies for the rational invader and guards using an Apollonius circle approach is presented in our previous research [33]. Since this is a zero-sum differential game with multiple agents, we are interested in knowing the elapsed time before the game reaches its equilibrium state. In Section 3.1.1, we described the five metrics for evaluating transfer learning. One of them is time to threshold, which is the elapsed time in the the target task for an agent to achieve a pre-specified performance level when transfer learning is applied. In our case, we choose the pre-specified performance level to be the game's equilibrium state. Therefore, we use a metric of simulation time steps per episode. This metric captures the trajectory of the elapsed time in each episode over the course of training. The simulation is run over 10 learning trials with 600 epochs per trial. We present the simulation time-steps per episode, averaged over 10 trials for the FRT and non-FRT case respectively in Fig. 4.18 and Fig. 4.19. 


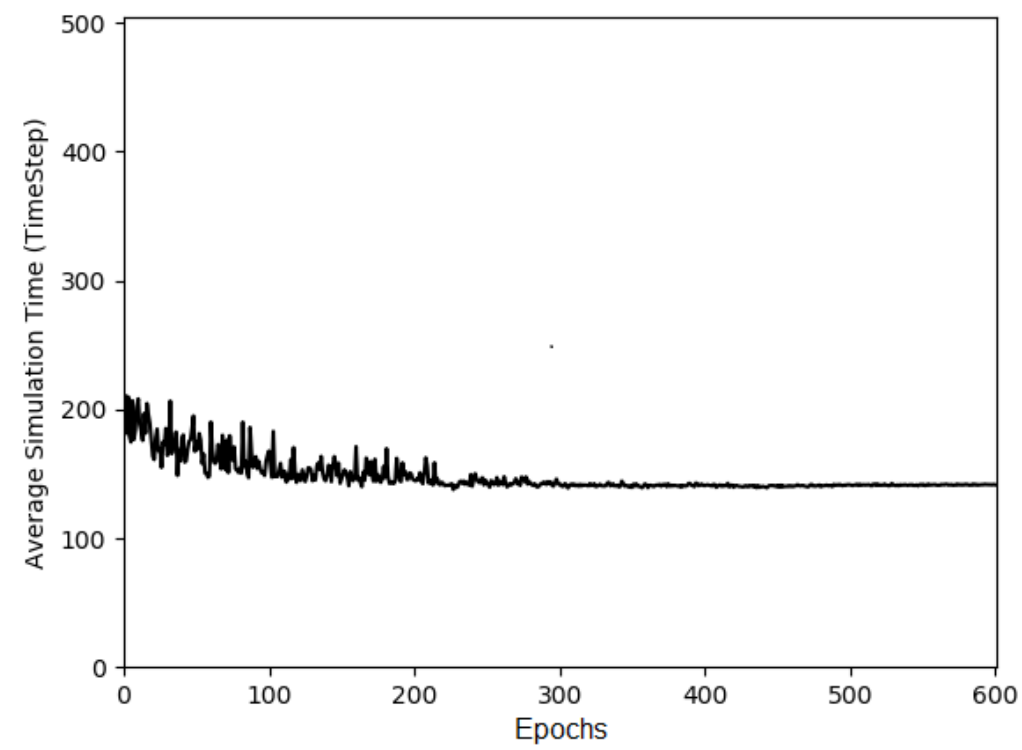

Figure 4.18: Simulation time-steps in 1 invader vs. 2 guards game when all agents are learning with FRT

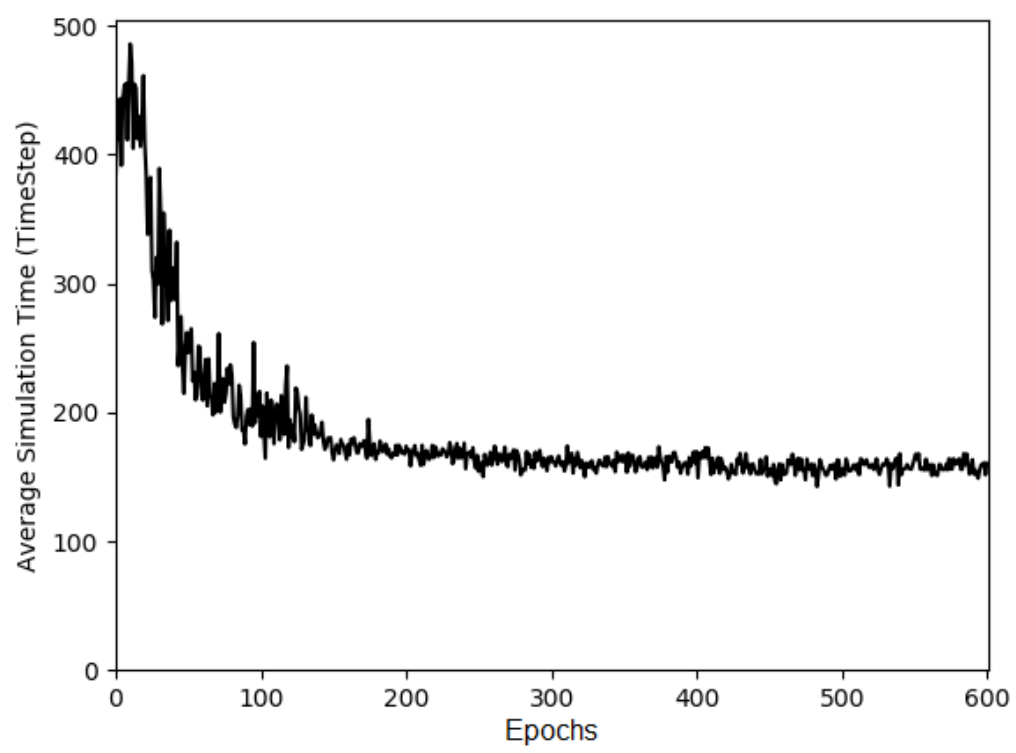

Figure 4.19: Simulation time-steps in 1 invader vs. 2 guards game when all agents are learning without FRT 


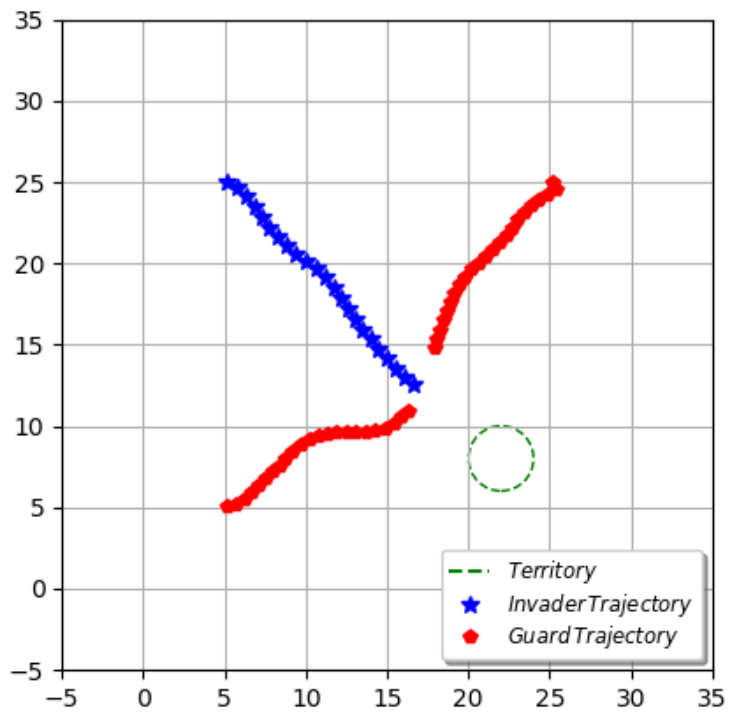

Figure 4.20: Epoch 300 in 1 invader vs. 2 guards game when all agents are learning with FRT

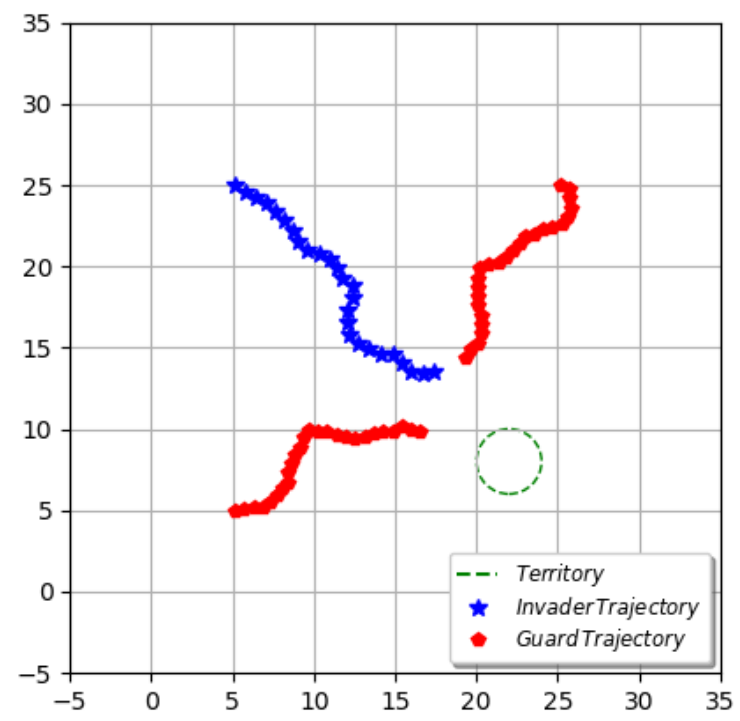

$\mathrm{S}$

Figure 4.21: Epoch 300 in 1 invader vs. 2 guards game when all agents are learning without FRT 
In the case when FRT is used, the equilibrium state of the differential game is reached much faster by the three competing agents who can learn their optimal strategies quickly. Similar to the multi-agent pursuit-evasion game in Section 4.2.1.2, the state space of the guard does not change between the source and target task. The final performance of the guards after 600 episode in the target task can be seen as a result of the training after 1200 episodes, with 600 episodes from the source task and a configuration (learning rate, exploration noise and discount factors) reset before the target task begins. Whereas the invader still requires to learn a new state space. Therefore, in this case the transfer benefit the guards more than it does for the invader. However, with the aid of FRT the invader is able to learn going toward the territory as a straight line more quickly instead of moving arbitrarily. This also leads to a faster capture from the guards' perspective. Hence the game is finished quickly and we see fewer time steps for each episode in Fig. 4.18. In comparison for the game without FRT, the agents tend to struggle in the beginning then slowly converge to their optimal strategies. This explains the reason we see more time steps per episode in Fig. 4.19, especially in the beginning of the game. In addition, we present the game trajectory of the agent's movement at 300 epochs in both scenarios in Fig. 4.20 and Fig. 4.21. As illustrated in the figures the performance of the agents are much better at 300 epochs when FRT is used.

\subsection{Summary}

In this Chapter, we investigated the possibility to transfer knowledge between related tasks that use the FACL algorithm. In order to enable knowledge transfer, we proposed the fuzzy rule transfer (FRT)method which maps the consequent parameters between the source and target task based on rules similarity. We showed the FRT 
method and the concept of the similarity between fuzzy rules in Section 4.1 with a detailed example given in Section 4.1.2. We conducted simulations in two sets of differential games; the pursuit-evasion game and the guarding-a-territory game in Section 4.2. In all simulations, the results showed the FRT method is able to speed up the learning process of the FACL agents in the target tasks. 


\section{Chapter 5}

\section{Conclusion}

Reusing knowledge from previous experience to accelerate learning in the current task is the central idea in transfer learning. This notion also applies to reinforcement learning where the agents acquire knowledge via interacting with the environment. In this thesis, we investigate the possibility of using transfer learning in the fuzzy reinforcement learning domain. A novel approach is proposed to transfer knowledge among similar tasks that use the fuzzy actor-critic learning (FACL) algorithm. We also introduced a fuzzy rule transfer (FRT) method specifically for processing the mapping of the fuzzy rules among the source and target tasks. We made an assumption that the target task is related to the source task but is represented by more complex states. Our method is fully implemented and tested in the domain of differential games where the state space and the action space are continuous.

We evaluate the proposed FRT algorithm in two sets of differential games; the pursuit-evasion game and the guarding-a-territory game. Our test domain also covers both the single-agent learning and the multi-agent learning scenarios. The results show that the agents are able to learn and achieve asymptotic performance faster in the target task when fuzzy rule transfer is applied. To our knowledge, this is the first

work to investigate the possibility of using transfer learning in the fuzzy reinforcement 
learning domain. We would like to reiterate the main achievement of this thesis as follows:

1. We investigated the possibility of using transfer learning in the fuzzy reinforcement learning domain.

2. We proposed a novel approach to achieve the goal of knowledge transfer among tasks that use FACL algorithm. We have also showed a detailed transfer learning example of inter-task mapping in the Paddle Game in Section 3.4.

3. We designed a fuzzy rule transfer method (FRT) to promote knowledge transfer between the source and target task by mapping the fuzzy rules between the FACL agents. We presented the method with detailed examples focusing on the key concepts including the similarity in fuzzy rules.

4. We evaluated our method in the two sets of differential games; the pursuitevasion game and the guarding-a-territory game. We also present how the games are designed and how the FRT is applied with further details. Additionally, the simulation covered both the single-agent learning and the multi-agent learning scenarios.

One direction of our future work will focus on how fuzzy rules from the source tasks can be selectively transferred. Currently we transfer knowledge of all fuzzy rules from a source task to a target task. Some of the rules can be extremely relevant and useful. However, some of the rules can be not so good or can even cause disturbance 
in a new task. How can we distinguish these sets of rules? If we are able to transfer the knowledge in fuzzy rules selectively, can we do better than the current solution? Another direction of our future work is combining multiple source tasks such that more experience can be transferred. So far we only use a single source task in the transfer. If we transfer the knowledge from multiple sources tasks, will it lead to a better generalization for the learning in a target task? These are some interesting questions that need to be answered. 


\section{List of References}

[1] R. S. Sutton and A. G. Barto, Reinforcement Learning : An Introduction. Cambridge, Massachusetts: MIT Press, 1998.

[2] H. M. Schwartz, Multi-Agent Machine Learning: A Reinforcement Approach. John Wiley and Sons, 2014.

[3] L. Jouffe, "Actor-critic learning based on fuzzy inference system," in Proceedings of the IEEE International Conference on Systems, Man and Cybernetics, vol. 1, 1996, pp. 339-344.

[4] L.-X. Wang, A course in fuzzy systems and control. Upper Saddle River, New Jersey: Prentice Hall, 1997.

[5] M. Andrecut and M. K. Ali, "Fuzzy reinforcement learning," International Journal of Modern Physics C, vol. 13, no. 05, pp. 659-674, 2002.

[6] M. E. Taylor and P. Stone, "Behavior transfer for value-function-based reinforcement learning," in Proceedings of the fourth international joint conference on Autonomous agents and multiagent systems, 2005, pp. 53-59.

[7] _ - "Transfer learning for reinforcement learning domains: A survey," Journal of Machine Learning Research, vol. 10, no. Jul, pp. 1633-1685, 2009.

[8] M. E. Taylor, P. Stone, and Y. Liu, "Transfer learning via inter-task mappings for temporal difference learning," Journal of Machine Learning Research, vol. 8, no. Sep, pp. 2125-2167, 2007.

[9] M. E. Taylor, S. Whiteson, and P. Stone, "Transfer via inter-task mappings in policy search reinforcement learning," in Proceedings of the 6th international joint conference on Autonomous agents and multiagent systems. ACM, 2007, p. 37 . 
[10] C. J. Watkins, "Learning from delayed rewards," Ph.D. dissertation, University of Cambridge, England, 1989.

[11] C. J. Watkins and P. Dayan, "Q-learning," Machine learning, vol. 8, no. 3-4, pp. 279-292, 1992.

[12] V. Mnih, K. Kavukcuoglu, D. Silver, A. Graves, I. Antonoglou, D. Wierstra, and M. Riedmiller, "Playing atari with deep reinforcement learning," arXiv preprint arXiv:1312.5602, 2013.

[13] L.-X. Wang, A course in fuzzy systems and control. Prentice-Hall, Inc., 1996.

[14] R. Razavi, S. Klein, and H. Claussen, "Self-optimization of capacity and coverage in lte networks using a fuzzy reinforcement learning approach," in 21st Annual IEEE International Symposium on Personal, Indoor and Mobile Radio Communications. IEEE, 2010, pp. 1865-1870.

[15] S. N. Givigi, H. M. Schwartz, and X. Lu, "A reinforcement learning adaptive fuzzy controller for differential games," Journal of Intelligent and Robotic Systems, vol. 59, no. 1, pp. 3-30, 2010.

[16] S. F. Desouky and H. M. Schwartz, "Self-learning fuzzy logic controllers for pursuit-evasion differential games," Robotics and Autonomous Systems, vol. 59, no. 1, pp. 22-33, 2011.

[17] X. Dai, C.-K. Li, and A. B. Rad, "An approach to tune fuzzy controllers based on reinforcement learning for autonomous vehicle control," IEEE Transactions on Intelligent Transportation Systems, vol. 6, no. 3, pp. 285-293, 2005.

[18] M. D. Awheda and H. M. Schwartz, "A decentralized fuzzy learning algorithm for pursuit-evasion differential games with superior evaders," Journal of Intelligent 63 Robotic Systems, vol. 83, no. 1, pp. 35-53, 2016.

[19] H. Schwartz, "An object oriented approach to fuzzy actor-critic learning for multi-agent differential games," in 2019 IEEE Symposium Series on Computational Intelligence (SSCI). IEEE, 2019, pp. 183-190.

[20] S. N. Givigi, H. M. Schwartz, and X. Lu, "An experimental adaptive fuzzy controller for differential games," in 2009 IEEE International Conference on Systems, Man and Cybernetics. IEEE, 2009, pp. 3017-3023. 
[21] F. L. Da Silva, R. Glatt, and A. H. R. Costa, "Simultaneously learning and advising in multiagent reinforcement learning," in Proceedings of the 16th conference on autonomous agents and multiagent systems, 2017, pp. 1100-1108.

[22] J. Ramon, K. Driessens, and T. Croonenborghs, "Transfer learning in reinforcement learning problems through partial policy recycling," in European Conference on Machine Learning. Springer, 2007, pp. 699-707.

[23] M. E. Taylor and P. Stone, "Cross-domain transfer for reinforcement learning," in Proceedings of the 24 th international conference on Machine learning. ACM, 2007, pp. 879-886.

[24] D. Silver, J. Schrittwieser, K. Simonyan, I. Antonoglou, A. Huang, A. Guez, T. Hubert, L. Baker, M. Lai, A. Bolton et al., "Mastering the game of go without human knowledge," Nature, vol. 550, no. 7676, pp. 354-359, 2017.

[25] M. Asada, S. Noda, S. Tawaratsumida, and K. Hosoda, "Vision-based behavior acquisition for a shooting robot by using a reinforcement learning," in Proc. of IAPR/IEEE Workshop on Visual Behaviors. Citeseer, 1994, pp. 112-118.

[26] D. Andre and A. Teller, "Evolving team darwin united," in Robot Soccer World Cup. Springer, 1998, pp. 346-351.

[27] I. Noda, H. Matsubara, K. Hiraki, and I. Frank, "Soccer server: A tool for research on multiagent systems," Applied Artificial Intelligence, vol. 12, no. 2-3, pp. 233-250, 1998.

[28] M. Chen, K. Dorer, E. Foroughi, F. Heintz, Z. Huang, S. Kapetanakis, K. Kostiadis, J. Kummeneje, J. Murray, I. Noda et al., "Robocup soccer server," Manual for Soccer Server Version, vol. 7, 2001.

[29] J. S. Albus, Brains, behavior, and robotics. Byte books Peterborough, NH, 1981.

[30] P. Stone, R. S. Sutton, and G. Kuhlmann, "Reinforcement learning for robocup soccer keepaway," Adaptive Behavior, vol. 13, no. 3, pp. 165-188, 2005.

[31] P. S. Foundation. turtle — turtle graphics. [Online]. Available: https: //docs.python.org/3.3/library/turtle.html?highlight=turtle 
[32] L. Jouffe, "Fuzzy inference system learning by reinforcement methods," IEEE Transactions on Systems, Man, and Cybernetics, Part C (Applications and Reviews), vol. 28, no. 3, pp. 338-355, 1998.

[33] C. V. Analikwu and H. M. Schwartz, "Multi-agent learning in the game of guarding a territory," Int. J. Innov. Comput. Inf. Control, vol. 13, no. 6, pp. 1855-1872, 2017.

[34] R. Isaacs, "A mathematical theory with applications to warfare and pursuit, control and optimization," 1965. 